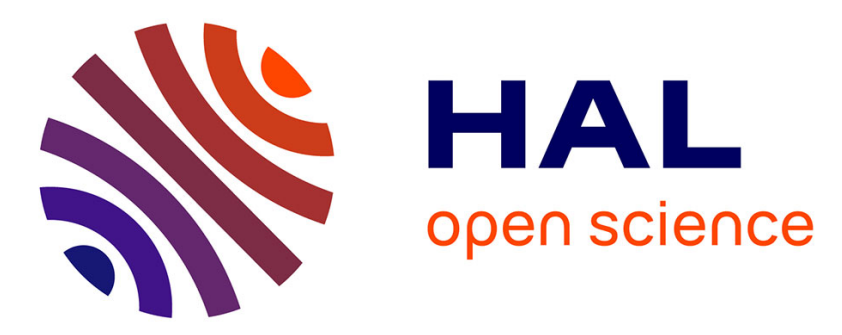

\title{
Direct Numerical Simulations of high Karlovitz number premixed flames for the analysis and modeling of the displacement speed
}

\author{
Edouard Suillaud, Karine Truffin, Olivier Colin, Denis Veynante
}

\section{To cite this version:}

Edouard Suillaud, Karine Truffin, Olivier Colin, Denis Veynante. Direct Numerical Simulations of high Karlovitz number premixed flames for the analysis and modeling of the displacement speed. Combustion and Flame, 2022, 236, pp.111770. 10.1016/j.combustflame.2021.111770 . hal-03438334

\author{
HAL Id: hal-03438334 \\ https://hal.science/hal-03438334
}

Submitted on 21 Nov 2021

HAL is a multi-disciplinary open access archive for the deposit and dissemination of scientific research documents, whether they are published or not. The documents may come from teaching and research institutions in France or abroad, or from public or private research centers.
L'archive ouverte pluridisciplinaire HAL, est destinée au dépôt et à la diffusion de documents scientifiques de niveau recherche, publiés ou non, émanant des établissements d'enseignement et de recherche français ou étrangers, des laboratoires publics ou privés. 


\title{
Direct Numerical Simulations of high Karlovitz number premixed flames for the analysis and modeling of the displacement speed.
}

\author{
Edouard Suillaud $^{\mathrm{a}, *}$, KKarine Truffin $^{\mathrm{a}}$, Olivier Colin $^{\mathrm{a}}$, Denis Veynante ${ }^{\mathrm{b}}$ \\ ${ }^{a}$ IFP Energies nouvelles, 1 et 4 avenue de Bois-Préau, 92852 Rueil-Malmaison, France ; Institut Carnot IFPEN Transports Energie \\ ${ }^{b}$ Ecole CentraleSupelec, Laboratoire EM2C, CNRS, Paris, France
}

\begin{abstract}
An a priori model for premixed turbulent flame combustion in the thin reaction zone (TRZ) regime is presented. This a priori model is deduced from the analysis of data from a series of direct numerical simulations (DNS) of stoichiometric $(\phi=1.0)$ premixed turbulent iso-octane flames, with Karlovitz number ranging from 2.9 to 46.2 . For each case two flames are considered: one with unity Lewis numbers to isolate the effect of turbulence on the flame, and one with non-unity Lewis numbers to study the influence of differential diffusion. First the reaction zone is shown to remain thin for each flame, leading to focus this study on a specific iso-surface in the reaction zone and how it is affected by turbulence. Second, the displacement speed $S_{d}$ on this iso-surface shows a differentiate dependency on tangential strain rate and curvature. This dependency is modeled through an expression of $S_{d}$ formally similar to the ones used in laminar flame theories, but using two effective turbulent Markstein lengths in place of the laminar ones. These lengths are shown to depend on the Lewis number and to decrease when the Karlovitz number increases, in agreement with previous studies showing a reduction of the effective Lewis number with the Karlovitz. From these DNS, an extension of the coherent flame model (CFM) to the TRZ regime is proposed, using a fine-grained flame surface density (FSD) located in the reaction zone. Models for the displacement speed, the tangential strain rate, and the stretch due to curvature are proposed. The a priori evaluation of these closures shows a significant improvement compared to the flamelet formulations.
\end{abstract}

Keywords: High Karlovitz, Direct Numerical Simulation (DNS), Combustion modeling, Turbulent premixed flame, Differential diffusion

\section{Introduction}

The challenges associated to the improvement of vehicle power-train efficiency and the deployment of ultra-low carbon technologies are presently driving a significant amount of research. The increase of engine efficiency, while preventing abnormal combustion (knock and pre-ignition), can be obtained by increasing the level of dilution and developing new aerodynamic strategies allowing to increase the turbulence intensity at spark-timing.

Operating with diluted combustion using burnt gases increases the engine efficiency while maintaining low pollutant emissions (e.g. NOx). Nevertheless, fundamental issues are raised in terms of combustion regimes. Indeed, diluted premixed flames tend to be thicker and

\footnotetext{
${ }^{*}$ Corresponding author:

Email address: edouard.suillaud@gmail.com (Edouard Suillaud)
}

propagate slower due to the lower flame temperature and larger dilution.

A thicker and slower flame is more sensitive to stirring by small turbulence structures. As a consequence the flame shifts from the flamelet to the thin reaction zone (TRZ) regime, where the smallest turbulent scales are able to penetrate and thicken the preheat zone without impacting the reaction zone as described by Peters in the combustion regime diagram [1]. This shift is well illustrated by Mounaïm-Rousselle et al. [2]. This change of regime can be measured by the Karlovitz number $K a$ comparing flame $\left(\tau_{f}\right)$ and Kolmogorov $\left(\tau_{\eta}\right)$ time-scales:

$$
K a=\frac{\tau_{f}}{\tau_{\eta}}=\left(\frac{\delta_{L}}{\eta}\right)^{2},
$$

where $\delta_{L}$ and $\eta$ are the flame thickness and Kolmogorov length-scales, respectively. The TRZ regime is then defined by $K a>1$. 
Experimentally, premixed flames in the TRZ regime have been studied for a long time. Lipatnikov and Chomiak [3] present a review of such experiments, which shows that preferential diffusion plays a major role on the turbulent flame speed $S_{T}$, not only in the flamelet regime, but also in the TRZ regime. This effect is measured by the Lewis number $L e$ of species $k$ defined as the ratio of the thermal diffusivity $D_{t h}$ to the mass diffusivity $D_{k}$ such that $L e=D_{t h} / D_{k}$. Both in the flamelet and in the TRZ regime, flames with $L e<1$ show a larger $S_{T}$ compared to flames with $L e>1$ for the same turbulent conditions and laminar flame speed [4, 5]. Preferential diffusion also influences the rate of $S_{T}$ increase with $u^{\prime}$ and also the level of $u^{\prime}$ at which the so-called bending of $S_{T}$ appears [4, 6-14], prior to the eventual extinction of the flame. Gülder and Smallwood [9] and then Nivarti et al. [15] deduced from experiments [8, 16] that while the increase of $S_{T}$ in the flamelet regime is essentially due to an increase of flame surface, in the TRZ regime this increase might be primarily due to an increase of the consumption speed $S_{L}$. This increase of $S_{L}$ is explained by the additional diffusivity brought by turbulent eddies smaller than the flame thickness $\delta_{L}$ in the preheat zone [15]. On the contrary, most DNS performed in the TRZ regime [6, 11, 17, 18] found an opposite trend: the average laminar flame speed remains close to $S_{L}^{0}$, while the flame surface keeps increasing. This difference could be explained by the difficulty to accurately measure flame surface and speed in such highly turbulent experiments, but it could also be explained by the artificial nature of the turbulence forcing technique in DNS. This question is still open and requires further research.

Usually, turbulent premixed flame combustion models are derived assuming a flamelet regime, where the flame is viewed as a collection of infinitely thin flamelets propagating locally at the laminar flame speed $S_{L}^{0}$, as described by Peters [19]. These models for large eddy simulations (LES) are based on various approaches, such as the thickened flame [20], the Gequation [1, 21] or the flame surface density (FSD) [2224], which is the amount of flame surface per unit volume. Different approaches were developed for the latter, both algebraic [25] or using a transport equation of the FSD [22]. However, these commonly used models fail to predict accurately the behavior of flames in the TRZ regime [10, 26], where the validity of the flamelet hypothesis is questioned because of the thickening of the preheat zone by the smallest turbulent eddies [1]. In addition, as reviewed in [3], these models also fail to account for the strong effect of preferential diffusion on $S_{T}$. An exception is the leading point approach in which the turbulent flame speed is piloted by the extremely stretched flame at the leading edge, thus making the flame response to stretch a first order parameter in the estimation of $S_{T}$. This approach has received recent support by the experimental study of Venkateswaran et al. [5].

In order to suppress the limitations of previously mentioned models, Peters [1] proposed a modified formulation of the G-equation for the TRZ regime. For this purpose he decomposed the displacement speed $S_{d}$ of the flame front on a particular species iso-surface, into two components. The first one, $S_{r}+S_{n}$, is the sum of the reaction and normal diffusion components of $S_{d}$, while the second one $-D \kappa$, where $\kappa$ is the flame curvature, is associated to tangential diffusion. This decomposition is then applied to the stretch by curvature $S_{d} K$ in the G-equation, leading to a new "diffusive" term $-D \kappa|\nabla G|$. This term is found to be dominant in the TRZ regime. In complement to Peters' work, Dave and Chaudhuri [27] proposed very recently to test the two Markstein lengths expression of $S_{d}$ derived in [28] for laminar flames, on turbulent flames located at the lower limit of the TRZ regime. They showed that, with a modified expression in which stretch by curvature $S_{d} \kappa$ is replaced by $S_{L}^{0} \kappa$, this expression agrees reasonably well with the DNS results, excepted on the burned gases side where flame surface annihilation leads to much larger $S_{d}$. In this latter situation, they proposed a new expression for $S_{d}$ which depends on temperature diffusivity and curvature, in qualitative agreement with the expression proposed by [1]. Another path towards the prediction of $S_{d}$ in turbulent flows is to derive and analyze the transport equation of $S_{d}$ as proposed in [29], with the objective to propose an adequate model of this quantity in the future.

The assumptions made by Peters in [1] are the starting point for high Karlovitz flames modeling in various studies on the subject. For instance, Hawkes and Chen [30] evaluated these assumptions using direct numerical simulations (DNS) of $\mathrm{CH}_{4}$-air and $\mathrm{H}_{2}$-air premixed flames interacting with decaying homogeneous isotropic turbulence (HIT). Different models were compared, such as a flamelet model, an asymptotic model and a model taking into account the tangential diffusion as suggested by Peters. The latter showed a priori significant improvement on the prediction of the flame stretch over the assumption of a constant flame speed. However, using the species diffusivity showed to be insufficient to correctly model the stretch of the flame and the authors concluded that further research was needed. This sensitivity to diffusion was well documented by Chakraborty et al. in [31-33] where, in the context of 
the FSD transport equation approach, DNS of statistically planar developing turbulent premixed flames with simplified chemistry and global Lewis numbers ranging from $L e=0.8$ to 1.2 were conducted. Chakraborty stated that the effect of differential diffusion on the displacement speed $S_{d}$ cannot be explained only with the tangential diffusion component. The influence of curvature on the displacement speed can be considered through a Markstein length, which should depend on the Lewis number, allowing to take into account differential diffusion. From these observations and the study by Han and Huh [34], which were confirmed by several studies such as [35-37], Katragadda et al. [38] proposed a model for the stretch due to curvature for flames in the TRZ regime. This model presents some encouraging results allowing to reproduce this stretch for different turbulent intensities.

Recently, Savard et al. in [18, 39, 43] brought some new insight on the TRZ regime and the role of preferential diffusion from the study of DNS of n-heptane turbulent premixed flames with and without unity Lewis numbers. Regarding the inner flame structure, DNS of Caltech [18, 39, 43] showed a decreasing role of preferential diffusion with increasing Karlovitz numbers, with an asymptotic behavior similar to that of a unity Lewis number flame. These authors interpreted this result as a progressive predominance of turbulent diffusion over molecular diffusion inside the preheat flame zone. However, no convergence between unity and non-unity Lewis flames was observed in terms of turbulent flame speeds, evidencing that preferential diffusion still plays a major role on $S_{T}$, even at the largest Karlovitz numbers. From the perspective of flame modeling, Savard and Blanquart [39] introduced a model for the effective Lewis number that takes into account the effect of the turbulence level through a Karlovitz number on preferential diffusion.

The aforementioned studies on the combustion analysis of the TRZ regime and a recent review by Driscoll et al. [44] highlighted major features of the flame characteristics (flame thickness, conditional flame profiles, etc...) in this regime but only a few a priori closures of the source terms needed for turbulent combustion models were proposed.

Coherent flame model (CFM) is a flamelet model based on transport equations for the progress variable $c$ and the flame surface density $\Sigma$. Closures for these equations were first proposed in RANS [45] and then in LES [22]. CFM has been extensively used in piston engine applications for more than two decades. While initially located in the flamelet regime, applications are increasingly located in the TRZ regime as explained previously. As CFM assumes that the flame is locally identical to a laminar unstretched flame (flamelet assumption), it explains why this model looses predictivity in this regime. The aim of the present study is to assess the possibility to extend the validity of CFM to the TRZ regime. For this purpose, a series of isooctane/air premixed flame DNS is presented and used to model a priori major source terms of the progress variable and flame surface density (FSD) equations in the TRZ regime. The major objectives of this work are as follows:

1. To analyse the flame characteristics in the TRZ regime in the context of the CFM approach, that is, in terms of progress variable and FSD.

2. To analyse the role of preferential diffusion which is known to be a major parameter, as observed in the present DNS, controlling the turbulent flame speed.

3. To propose a model for the flame displacement speed which enters the progress variable source term and stretch by curvature in the FSD equation.

4. To propose a model for the turbulent strain rate term in the FSD equation, which is the major source term of FSD.

The paper is organised as follows. The necessary mathematical background on CFM and details on the DNS set-up are given in sections 2 and 3 , respectively. Section 4 introduces the results, including an analysis and discussion on the displacement speed. Finally, the complete model is presented and compared to the DNS in section 5 .

\section{Mathematical Background}

The CFM approach describes the gases state thanks to a progress variable $c$, that goes from $c=0$ in the fresh gases to $c=1$ in the fully burnt gases. There exist several ways to define this variable, as discussed by Chakraborty and Cant in [33]. In the present study it is defined as the reduced temperature:

$$
c=\frac{T-T_{u}}{T_{b}-T_{u}}
$$

where subscripts $u$ and $b$ stand for unburned and burnt gases states, respectively. Note that some of the analysis carried out in this study were repeated with a progress variable defined thanks to the fuel mass fraction (not shown here), and showed similar results. This indicates that for the quantities studies, the choice of the progress variable is not fundamental. 
From Eq. (2) and from the conservation of total energy, a balance equation is derived for the progress variable $c$. Then, in the large eddy simulation (LES) context, this equation is space filtered [46] giving Eq. (3):

$$
\frac{\partial \overline{\rho c}}{\partial t}+\nabla \cdot(\bar{\rho} \widetilde{\mathbf{u} c})=-\underbrace{\nabla \cdot(\overline{\rho \mathbf{u} c}-\bar{\rho} \widetilde{\mathbf{u}})}_{T_{1}}+\nabla \cdot(\overline{\rho D \nabla c})+\overline{\dot{\omega}}_{c},
$$

$D$ being the thermal diffusivity $D=\lambda /\left(\rho C_{p}\right)$, with $\rho$ the density, $\lambda$ the thermal conductivity, $C_{p}$ the heat capacity at constant pressure and $\dot{\omega}_{c}$ the chemical source term. The filtered quantity $\bar{Q}$ is then defined as: $\overline{Q(\mathbf{x}, t)}=$ $\int_{\mathcal{V}} Q\left(\mathbf{x}^{\prime}, t\right) G_{\Delta}\left(\mathbf{x}-\mathbf{x}^{\prime}\right) d \mathbf{x}^{\prime}$, where $\mathcal{V}$ is the simulation domain, $\Delta$ the filter width and $\mathbf{x}$ the location vector. In finite volume codes the filter size is set to be the cell size and no explicit filtering is performed.

The displacement speed $S_{d}$ of each progress variable iso-surface relatively to the local flow velocity is often used to characterize the propagation of the flame front. In the present study $S_{d}$ is deduced from the exact balance equation of the progress variable $c$ expressed as:

$$
S_{d}=\frac{1}{\rho|\nabla c|} \frac{D \rho c}{D t} .
$$

Defining the generalized FSD [47] $\bar{\Sigma}=\overline{|\nabla c|}$, and the surface averaging operation $\langle Q\rangle_{s}=\overline{Q|\nabla c|} / \overline{|\nabla c|}$ where $Q$ is a general quantity, the right hand side (RHS) term in Eq. (3) is written:

$$
\nabla \cdot(\overline{\rho D \nabla c})+\overline{\dot{\omega}}_{c}=\overline{\rho S_{d}|\nabla c|}=\underbrace{\left\langle\rho S_{d}\right\rangle_{s} \bar{\Sigma}}_{T_{2}} .
$$

The closure of the RHS term in Eq. (5) requires the modeling of $\left\langle\rho S_{d}\right\rangle_{s}$ and of the filtered FSD. In the CFM approach, the filtered FSD is computed through a transport equation as proposed in [24, 25]:

$$
\frac{\partial \bar{\Sigma}}{\partial t}+\nabla \cdot(\widetilde{\mathbf{u}} \bar{\Sigma})=-\underbrace{\nabla \cdot\left(\langle\mathbf{u}\rangle_{s} \bar{\Sigma}-\widetilde{\mathbf{u}} \bar{\Sigma}\right)}_{T_{3}}+\underbrace{\langle\nabla \cdot \mathbf{u}-\mathbf{n n}: \nabla \mathbf{u}\rangle_{s, c^{*}} \bar{\Sigma}}_{T_{4}}
$$$$
+\underbrace{\left\langle S_{d} \nabla \cdot \mathbf{n}\right\rangle_{s} \bar{\Sigma}}_{T_{5}}-\underbrace{\nabla \cdot\left(\left\langle S_{d} \mathbf{n}\right\rangle_{S}^{\text {decomposed }}{ }_{\pi}+S_{t}\right. \text {. These terms correspond to the contributions of }}_{T_{6}}
$$

where $\mathbf{n}=-\nabla c /|\nabla c|$ is the local flame normal vector pointing towards the reactants, $\langle\nabla \cdot \mathbf{u}-\mathbf{n n}: \nabla \mathbf{u}\rangle_{s}$ is the tangential strain rate acting on the flame surface which will be called $\left\langle a_{T}\right\rangle_{s}$ in the following, $\left\langle S_{d} \nabla \cdot \mathbf{n}\right\rangle_{s}$ is the stretch due to curvature and $\nabla \cdot\left(\left\langle S_{d} \mathbf{n}\right\rangle_{s} \bar{\Sigma}\right)$ is a propagation term.
From Eqs. (3) and (6), six terms need to be closed:

1. The turbulent transport $T_{1}=\nabla \cdot(\overline{\rho \mathbf{u} c})-\nabla \cdot(\bar{\rho} \widetilde{\mathbf{u}})$.

2. The displacement speed weighted by the density $\left\langle\rho S_{d}\right\rangle_{s}$ in $T_{2}=\left\langle\rho S_{d}\right\rangle_{s} \bar{\Sigma}$

3. The turbulent transport of FSD $T_{3}=\nabla$. $\left(\langle\mathbf{u}\rangle_{s} \bar{\Sigma}-\widetilde{\mathbf{u}} \bar{\Sigma}\right)$

4. The tangential strain rate $\left\langle a_{T}\right\rangle_{s}$ in $T_{4}=\left\langle a_{T}\right\rangle_{s} \bar{\Sigma}$

5. The stretch due to curvature $\left\langle S_{d} \nabla \cdot \mathbf{n}\right\rangle_{s}$ in $T_{5}=$ $\left\langle\rho S_{d} \nabla \cdot \mathbf{n}\right\rangle_{s} \bar{\Sigma}$

6. The propagation term $T_{6}=\nabla \cdot\left(\left\langle S_{d} \mathbf{n}\right\rangle_{s} \bar{\Sigma}\right)$

Many studies [1, 18, 30, 34, 36, 37, 39.43][Wang.2017] suggest that the key parameters to be modeled at high turbulence intensities are terms $T_{2}, T_{4}$ and $T_{5}$, which are the focus of this study. The turbulent transport term $T_{1}$ is commonly closed under a gradient assumption and is written as a turbulent diffusion. Rymer [48] showed that in laminar cases $T_{1}$ is non zero and tends to $-\rho_{u} S_{L}^{0} \nabla$. $[(\bar{c}-\widetilde{c}) \overline{\mathbf{n}}]$. Based on this consideration and on the work of Richard et al. [22], the following closures for $T_{1}, T_{3}$ and $T_{6}$ will be retained in a first step:

$$
\begin{gathered}
T_{1}=-\nabla \cdot\left[\bar{\rho} \frac{v_{t}}{S_{c t}} \nabla \widetilde{c}\right]-\rho_{u} S_{L}^{0} \nabla \cdot[(\bar{c}-\widetilde{c}) \overline{\mathbf{n}}] \\
T_{3}=-\nabla \cdot\left(\frac{v_{t}}{S_{c t}} \nabla \bar{\Sigma}\right)+\nabla \cdot\left(\tau S_{L}^{0} \widetilde{c}\langle\mathbf{n}\rangle_{s} \bar{\Sigma}\right), \\
T_{6}=\nabla \cdot\left(\frac{\rho_{u}}{\bar{\rho}} S_{L}^{0}\langle\mathbf{n}\rangle_{s} \bar{\Sigma}\right),
\end{gathered}
$$

where $v_{t}$ and $S_{c t}$ are the turbulent viscosity and a turbulent Schmidt number, respectively. $\tau=\rho_{u} / \rho_{b}-1$ is the thermal expansion rate across the flame front and $\rho_{b}$ is the burned gases density. $T_{6}$ does not act as a source term but it describes the planar propagation of iso-surfaces of the progress variable at the laminar flame speed with a correction to take into account thermal expansion across the flame front. This term becomes negligible in very turbulent flows.

The displacement speed $S_{d}$ given by Eq. (4) can be reaction $S_{r}$, normal diffusion $S_{n}$ and tangential diffusion $S_{t}$, respectively, and are defined as:

$$
\begin{aligned}
& S_{r}=\frac{\dot{\omega}_{c}}{\rho|\nabla c|}, \\
& S_{n}=-\frac{1}{\rho|\nabla c|} \mathbf{n} \cdot \nabla(\rho D|\nabla c|), \\
& S_{t}=-D \kappa,
\end{aligned}
$$


where $\kappa=\nabla \cdot \mathbf{n}$ measures the local flame curvature. The statistical behavior of the three components of the displacement speed are discussed in detail in section 4.5. while models for the displacement speed $\left(T_{2}\right)$ and for the stretch due to curvature $\left(T_{5}\right)$ are proposed and discussed in section 5.1

\section{Direct numerical simulation set-up}

For this study, the DNS computations were conducted using the AVBP code [50], which was already used for DNS in [51]. It solves the three-dimensional compressible Navier-Stokes equations on unstructured and hybrid meshes. The AVBP numerical schemes are based on the cell-vertex method. The convective scheme used in this study is the finite-volume Lax-Wendroff scheme (LW), $2^{\text {nd }}$ order in space and time. In order to make sure the limited order of LW does not alter the flame statistics, one simulation was run with the $3^{\text {rd }}$ order space and time finite-element Tayor-Galerkin scheme [52]. As no significant difference in the flame statistics was observed, the LW scheme was preferred for its lower CPU cost. The flow configuration is first introduced with a description of the chemistry. Then, the turbulence forcing method is described. Finally, the different cases simulated are presented.

\subsection{Flow configuration and chemistry}

Figure 1 presents a schematic view of the computational domain, which is similar to that of Savart et al. [39, 41] or Aspden et al. [53, 54]. A statisticallyflat flame interacting with a turbulent field is chosen, using a forcing method described in section 3.2 In this configuration the inlet and outlet boundaries are specified in the direction of the mean flame propagation. The transverse boundaries are considered to be periodic. The outflow boundary is taken to be partially non-reflecting and specified according to the NavierStokes Characteristic Boundary Conditions (NSCBC) technique [55]. For each simulation, the inflow velocity $U_{\text {in }}$ is constant throughout the simulation and selected to match the turbulent flame speed $S_{T}$. In practice, the flame drifts slightly, but the velocity of the drift $U_{\text {in }}-S_{T}$ is small and constant in average. This allows to compute the flame statistics considering at each instant $t$ the space origin as $x_{0}(t)=x_{0}(t=0)+\left(U_{i n}-S_{T}\right) t$.

The domain width is chosen as $L \approx 15 \delta_{L}$, where $\delta_{L}=\left(T_{b}-T_{u}\right) / \max (|\nabla T|)$ is the thermal flame thickness with $T_{u}$ and $T_{b}$ the temperature in the fresh and burnt gases, respectively. The cell size $\Delta_{x}$ used is chosen for each simulation as the minimum length allowing to get at least 20 grid points within the thermal thickness and to get at least a length ratio $\eta_{k} / \Delta_{x} \geq 0.5$ (where $\eta_{k}$ is the Kolmogorov length scale).

The iso-octane/air kinetics are described by a two-step Arrhenius mechanism with 6 species:

$$
\begin{aligned}
\mathrm{C}_{8} \mathrm{H}_{18}+8.5 \mathrm{O}_{2} & \rightarrow 8 \mathrm{CO}+9 \mathrm{H}_{2} \mathrm{O}, \\
\mathrm{CO}+\frac{1}{2} \mathrm{O}_{2} & \rightleftharpoons \mathrm{CO}_{2} .
\end{aligned}
$$

This scheme was developed for piston engine simulations and validated using both the laminar flame speed $S_{L}^{0}$ and the laminar flame thickness $\delta_{L}^{0}$ for the following fresh gases conditions: $323 \mathrm{~K}$, a constant pressure of $1 \mathrm{~atm}$ and an equivalence ratio ranging between 0.5 and 1.2 [56]. The forward reaction rates $k_{f 1}$ and $k_{f 2}$ for $\mathrm{C}_{8} \mathrm{H}_{18}$ oxidation and for $\mathrm{CO}-\mathrm{CO}_{2}$ equilibrium, respectively, are expressed below.

$$
\begin{gathered}
k_{f 1}=A_{1} T^{b_{1}} e^{-\frac{E_{a, 1}}{R T}}\left[C_{8} H_{18}\right]^{n_{C_{8} H_{18}}}\left[O_{2}\right]^{n_{O_{2}, 1}}, \\
k_{f 2}=A_{2} T^{b_{2}} e^{-\frac{E_{a, 2}}{R T}}[C O]^{n_{C O}}\left[O_{2}\right]^{n_{O_{2}, 2}} .
\end{gathered}
$$

$A_{i}$ is the pre-exponential factor, $b_{i}$ is the temperature exponent, $E_{a, i}$ is the activation energy of reaction $i$, and $n_{k, i}$ are the reaction exponents for species $k$ in reaction $i$ (Table 1). $R$ is the ideal gas constant equal to $8.314 \mathrm{~J} . \mathrm{K}^{-1} \cdot \mathrm{mol}^{-1}$.

Using a simplified mechanism with only few species might alter the effect of differential diffusion. For this reason, the influence of the chemical mechanism was investigated by performing a simulation of case B (see below for cases description) with an analytically reduced chemistry (ARC) mechanism: from a reduced synthetic paraffinic kerosene (SPK) mechanism (48 species and 416 reactions) offering the possibility to model lean premixed combustion of air and isooctane, a reduction procedure was applied using the YARK tool [57] which includes skeletal reductions and quasi-steady state (QSS) approximations. The final mechanism, called ISOOCT18, contains 18 transported species and 13 QSS species [58]. It has been validated for the following fresh gases conditions: temperature $300-450 \mathrm{~K}$, equivalent ratio 0.7-1.0 and ambient pressure of $1 \mathrm{~atm}$. Validation was performed using laminar flame speeds computed with the SPK mechanism [59] using Cantera.

Finally, the effect of preferential diffusion in the TRZ regime is investigated conducting simulations twice for each turbulence level, as proposed in Savard et al. in [18, 39, 42]: once considering differential diffusion with non-unity Lewis numbers $(L e>1)$ and once 


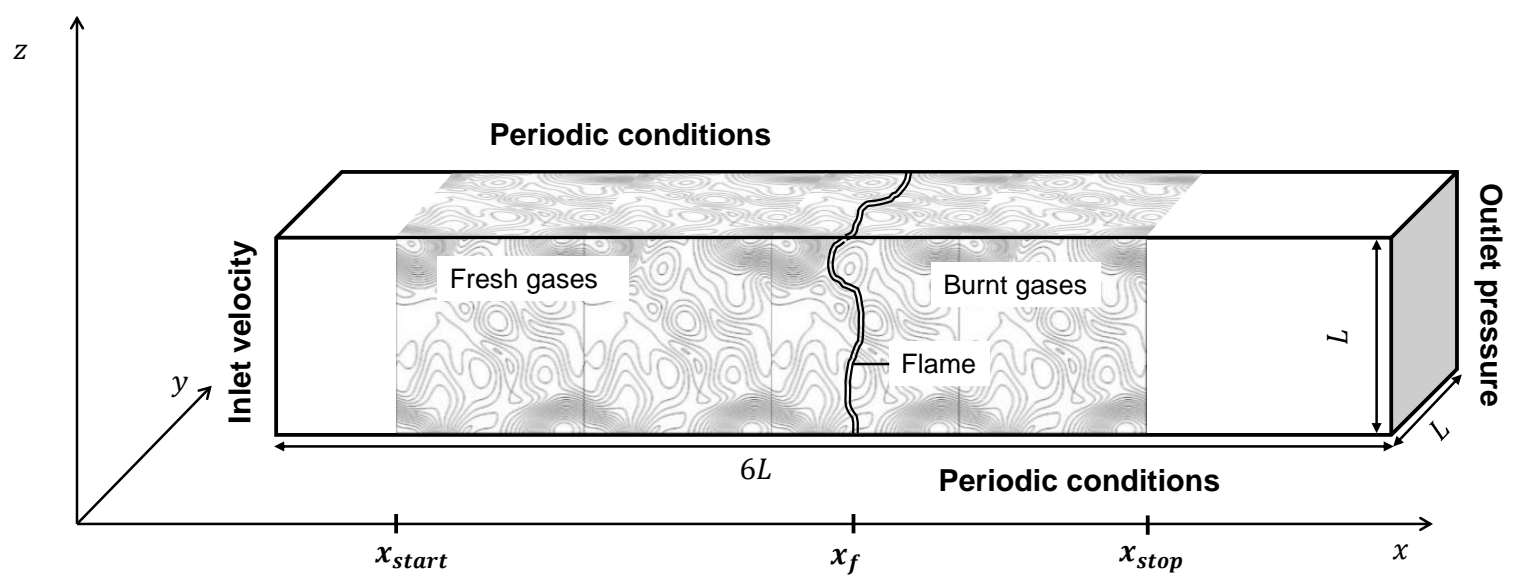

Figure 1: Schematic view of the flow configuration of the DNS for forced turbulence simulations.

Table 1: Arrhenius parameters for the $\mathrm{C}_{8} \mathrm{H}_{18}$ 2-step mechanism. The activation energy $E_{a, i}$ is expressed in $[\mathrm{cal} / \mathrm{mol}]$.

\begin{tabular}{|c|c|c|c|c|c|c|}
\hline Case & $\mathbf{L e}_{\text {Fuel }}$ & reaction & $A_{i}$ & $b_{i}$ & $E_{a, i}$ & $n_{k}$ \\
\hline \multirow{2}{*}{ Le $>1$} & \multirow{2}{*}{2.9} & $\begin{array}{l}\mathrm{C}_{8} \mathrm{H}_{18} \\
\text { oxidation }\end{array}$ & $5.443 \cdot 10^{12}$ & 0.1 & $3.6 \cdot 10^{4}$ & $\begin{array}{l}n_{C_{8} H_{18}}=1.1 \\
n_{O_{2}, 1}=0.54\end{array}$ \\
\hline & & $\begin{array}{l}\mathrm{CO}-\mathrm{CO}_{2} \\
\text { equilibrium }\end{array}$ & $2.0 \cdot 10^{9}$ & 0 & $1.4 \cdot 10^{4}$ & $\begin{array}{l}n_{C O}=1.0 \\
n_{O_{2}, 2}=0.5\end{array}$ \\
\hline \multirow{3}{*}{$L e=1$} & \multirow{3}{*}{1.0} & $\mathrm{C}_{8} \mathrm{H}_{18}$ & $9.150 \cdot 10^{12}$ & 0.1 & $3.6 \cdot 10^{4}$ & $n_{C_{8} H_{18}}=1.1$ \\
\hline & & oxidation & & & & $n_{O_{2}, 1}=0.54$ \\
\hline & & $\begin{array}{l}\mathrm{CU}-\mathrm{CU}_{2} \\
\text { equilibrium }\end{array}$ & $3.327 \cdot 10^{9}$ & 0 & $1.4 \cdot 10^{4}$ & $\begin{array}{l}n_{C O}=1.0 \\
n_{O_{2}, 2}=0.5\end{array}$ \\
\hline
\end{tabular}

with the Lewis number for all the species set to unity $(L e=1)$. The same laminar flame speed and thickness for both cases were achieved adapting the Arrhenius coefficients of the 2-step mechanism for the $L e=1$ case as shown in Table 1 .

For the whole study, a stoichiometric $(\Phi=1) \mathrm{C}_{8} \mathrm{H}_{18}$ /air mixture is considered, at a temperature $T_{u}=300 \mathrm{~K}$ and a pressure $P_{u}=1 \mathrm{~atm}$, leading to a laminar flame speed $S_{L}^{0}=0.366 \mathrm{~m} / \mathrm{s}$ and a thermal flame thickness $\delta_{L}=\left(T_{b}-T_{u}\right) / \max (|\nabla T|)=385 \mu \mathrm{m}$. Note that the laminar flame thickness $\delta_{L}$ computed using ISOOCT18 mechanism is larger $(560 \mu \mathrm{m})$.

\subsection{Turbulence forcing method}

In this study turbulence is supposed to be homogeneous and isotropic. In the absence of velocity field forcing, the decay of the turbulent kinetic energy (TKE), $k$, can be estimated from theory considering $d k / d t=$ $-k / \tau_{t}$, with $\tau_{t}$ the eddy turnover time $\left(\tau_{t}=l_{t} / u^{\prime}\right.$ with $u^{\prime}$ the characteristic velocity of the integral scale and $l_{t}$ the longitudinal length of the integral scale). As reported in Table 2, the Damköhler number $D a=\tau_{t} / \tau_{f}$ (where $\tau_{f}=\delta_{L} / S_{L}^{0}$ is the flame characteristic time) is quite in- ferior to unity for most cases considered here. As a consequence, a decaying turbulence set-up would not allow the flame to adapt to turbulence, therefore leading to unrealistic statistics. For this reason, as done previously by many authors [39,-41, 53, 54], a forced turbulence is considered, allowing to reach a statistically steady state flame and thus meaningful flame statistics. In this study, a spectral forcing method proposed by Eswaran and Pope [60] is used to offset the decay of TKE and maintain the turbulence characteristics.

This method generates a stochastic time-evolving forcing vector $\mathbf{f}$. This vector, introduced as a source term in the momentum equation, $\rho \mathbf{f}$, generates energy at the largest scales of the domain. When the rate of energy introduced by the forcing technique equals the rate of dissipation, a statistically steady state is reached, with a full spectrum of scales ranging from the forcing one down to the Kolmogorov scale. However, this forcing term introduces some heating by viscous dissipation of the turbulent kinetic energy, which should be avoided. For this purpose a modified energy source term $S_{e}$ is defined, according to the study of Paoli and Shariff [61], 
with:

$$
S_{e}=\rho \mathbf{f} \cdot \mathbf{u}-\langle\rho \mathbf{f} \cdot \mathbf{u}\rangle_{V},
$$

where \langle\rangle$_{V}$ is the averaging operator over the whole domain.

With the forcing method used, HIT is imposed in the volume defined in Fig. 1 where the flame is contained. It can be argued that this forcing term being artificial and imposed at the flame location, this approach might lead to erroneous flame statistics. For this purpose, one additional simulation was performed suppressing the forcing for $c<0.05$, as suggested in [62]. This means that a large part of the preheat zone and the whole heat release region are free of forcing in these simulations. A comparison of the two forcing methods is presented in Appendix A

\subsection{Simulation parameters}

Three cases A, B and E are investigated with increasing Karlovitz number while keeping a nearly constant integral length scale. This point is important as it means that the flame will see the same largest scales, while the Kolmogorov scale decreases from A to E, therefore increasing the range of scales interacting with the flame structure.

The Karlovitz number, defined as $\mathrm{Ka}=$ $\left(u^{\prime} / S_{L}^{0}\right)^{3 / 2}\left(l_{t} / \delta_{L}\right)^{-1 / 2}$, varies from 2.9 to 46.2 as seen in Table 2 Case $\mathrm{A}$ is expected to be representative of the upper part of the flamelet regime. Cases B and E were selected to fall inside the TRZ regime. These cases are performed with differential diffusion $(L e>1)$ and the 2-step chemistry presented in section 3.1 According to Matalon et al. [63], the effective Lewis number of a stoichiometric mixture is $L e_{\text {mix }}=\left(L e_{O x}+L e_{F u l}\right) / 2$, where $L e_{O x}$ and $L e_{F u e l}$ are Lewis numbers of the oxidant and fuel, respectively. So, considering $L e_{O x}=1.1$ and $L e_{\text {Fuel }}=2.9$, the stoichiometric $\mathrm{C}_{8} \mathrm{H}_{18}$ /air mixture has an effective Lewis number $L e_{m i x}=2$. Cases $A_{1}$, $\mathrm{B}_{1}$ and $\mathrm{E}_{1}$ are performed with the same turbulence forcing parameters as $\mathrm{A}, \mathrm{B}$ and $\mathrm{E}$, but using unity Lewis numbers for all species $(L e=1)$ and the modified 2step chemistry. The integral length scale and the turbulence intensity reported in Table 2 are computed from the turbulent kinetic energy (TKE) $k$ and the dissipation rate $\varepsilon$, extracted from DNS averaging fresh gas characteristics: $k=\left\langle u_{i}^{\prime} u_{i}^{\prime}\right\rangle / 2=3 u^{\prime 2} / 2$ and $\varepsilon=2 v\left\langle S_{i j} \frac{\partial u_{i}^{\prime}}{\partial x_{j}}\right\rangle$ with $S_{i j}=\left(\frac{\partial u_{i}^{\prime}}{\partial x_{j}}+\frac{\partial u_{i}^{\prime}}{\partial x_{i}}\right)$. Assuming isotropic turbulence, the characteristic velocity of the integral scale is computed as $u^{\prime}=\sqrt{2 k / 3}$. Then, the integral length scale can be estimated as $l_{t}=u^{\prime 3} / \varepsilon$. This definition for the integral length scale is related to the longitudinal integral lengths $L_{i i}^{i}$ as follows [64]: $L_{i i}^{i}=\left(\beta n l_{t} / 3\right) \sqrt{2 / \pi}$, where $n$ is the dimension of the simulation equal to 3 , and $\beta=\pi / 2$ when $n=3$. This leads to $L_{i i}^{i} \approx 0.4 l_{t}$. As seen in Table 2 the turbulence intensity and integral length scale slightly differ between unity and non-unity Lewis number cases. Case B-ARC is identical to case $B$ but performed with the ARC mechanism in place of the simplified 2-step mechanism in order to evaluate the importance of the chemistry description on flame statistics. All these cases are represented with plain symbols in the Peters-Borghi diagram in Fig. 2 along with previous DNS of the literature.

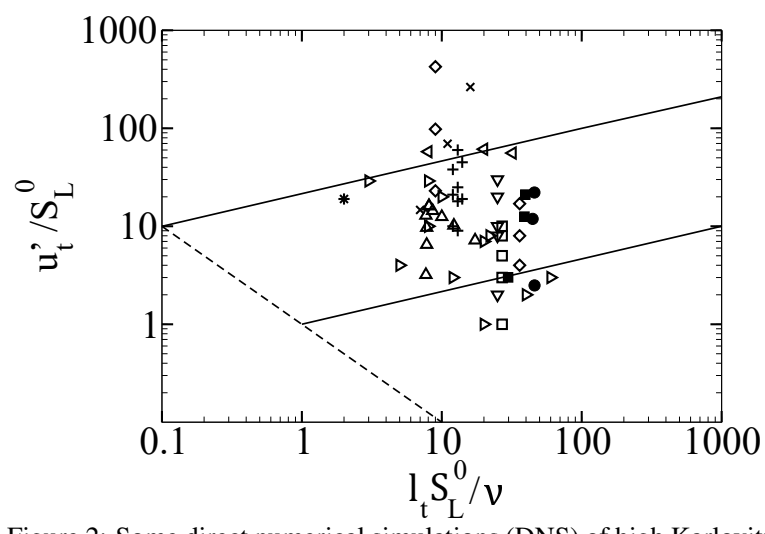

Figure 2: Some direct numerical simulations (DNS) of high Karlovitz turbulent premixed flames: $\square$ DNS from [11], $\diamond$ DNS from $[53,54$ 65. 68], $\triangle$ DNS from [34], $\triangleright$ DNS from [30 69], $\nabla$ DNS from [6], $\triangleleft$ DNS from [36], * DNS by [17, 70], and + DNS from [18 39, 43]. Plain symbols represent the DNS conducted in the current study: $L e>1$ and $\boldsymbol{\square} e=1$ cases.

\section{DNS analysis}

\subsection{Global flame properties}

Fig. 3 presents instantaneous color fields of the heat release rate $\left(\dot{\omega}_{T}^{\prime}\right)$ normalized by its maximum value and iso-contours of $c$ on two-dimensional slices in the $\mathrm{x}$ $\mathrm{y}$ mid-plane. The heat release rate is defined following [46] as $\dot{\omega}_{T}^{\prime}=-\sum_{k=1}^{N} h_{k} \dot{\omega}_{k}$ with $\dot{\omega}_{k}$ the reaction rate of species $k$ and $h_{k}$ the mass enthalpy of species $k$. For the unity Lewis number simulations, the heat release rate shows qualitatively weak variations along the flame front, even for cases $B_{1}$ and $E_{1}$. At the same time these cases present $c$ iso-contours that are not parallel to each other for $c$ values approximately smaller than 0.4 , corresponding to the preheat zone. An increase of the Karlovitz number (case $A_{1}$ to $E_{1}$ ) seems to slightly thicken the preheat zone when preferential diffusion is not accounted for. 
Table 2: The DNS set-up for the simulations of the interaction between a planar flame and forced homogeneous isotropic turbulence.

\begin{tabular}{lcccccccc}
\hline Case & $\mathbf{L e}_{\text {Fuel }}$ & $\mathbf{u}^{\prime} / \mathbf{S}_{\mathbf{L}}^{\mathbf{0}}$ & $\mathbf{I}_{\mathbf{t}} / \delta_{\mathbf{L}}$ & $\mathbf{R e}_{\mathbf{t}}$ & $\mathbf{K a}$ & $\mathbf{D a}$ & $\delta_{\mathbf{L}} / \boldsymbol{\Delta}_{\mathbf{x}}$ & $\eta_{\mathbf{k}} / \boldsymbol{\Delta}_{\mathbf{x}}$ \\
\hline $\mathrm{A}$ & 2.9 & 3.0 & 3.3 & 89.6 & 2.9 & 1.1 & 20 & 1.7 \\
$\mathrm{~B}$ & 2.9 & 12.5 & 4.3 & 489.0 & 21.4 & 0.3 & 20 & 0.6 \\
$\mathrm{E}$ & 2.9 & 21.1 & 4.4 & 833.2 & 46.2 & 0.2 & 31 & 0.7 \\
$\mathrm{~A}_{1}$ & 1.0 & 2.5 & 5.1 & 74.8 & 2.7 & 1.0 & 20 & 1.8 \\
$\mathrm{~B}_{1}$ & 1.0 & 11.9 & 4.9 & 530.7 & 18.5 & 0.4 & 20 & 0.7 \\
$\mathrm{E}_{1}$ & 1.0 & 22.1 & 5.1 & 1016.1 & 46.2 & 0.2 & 31 & 0.7 \\
B-ARC & 2.9 & 12.6 & 4.4 & 501.3 & 21.2 & 0.4 & 31 & 0.6 \\
\hline
\end{tabular}

subscript 1 designates simulation with unity Lewis numbers.

When differential diffusion is taken into account, the thickening of the flame front with increasing $K a$ is much more pronounced than in unity Lewis number cases. Indeed, for case A, which is at the upper limit of the flamelet regime $(K a=2.9)$, the contours of progress variable are wrinkled but remain parallel to each other. For cases B and E, which are in the TRZ regime $(K a=21.4$ and $K a=46.2$, respectively), $c$ iso-contours on the unburned gases side of the flame are qualitatively more wrinkled than the ones close to the burnt gases side. Moreover, they remain globally parallel to each other for $c$ values approximately larger than 0.7 , that is, in the heat release region. In addition, the fluctuations of heat release rate in cases $B$ and $\mathrm{E}$ are much larger than in cases $\mathrm{B}_{1}, \mathrm{E}_{1}$ and $\mathrm{A}$, where $\dot{\omega}_{T}^{\prime} / \dot{\omega}_{T}^{\prime \max }$ decreases down to 0.1 . Previous DNS in the literature [36, 37, 40, 42, 44, 53, 65, 66] also observed such events. The Klimov-Williams criterion states that for $K a>1$, flames are subject to extinction. As present regimes go well above $K a>1$, it is not surprising that locally in the turbulent flow, a strain rate larger than this quenching rate is observed and induces local extinctions.

Thus, this qualitative analysis shows that an increase of the turbulent intensity first increases the wrinkling of the flame. As already observed by many authors [39. 41, 53, 54], the second consequence is a thickening of the preheat zone of the flame, which appears more important when the differential diffusion is taken into account. In addition, turbulence also seems to impact the heat release rate by increasing its fluctuations, also observed by Savard et al. [40-42]. As for the thickening, this last effect is less important for unity Lewis numbers.

Figure 4 shows the temporal evolution of the flame surface defined as $\mathcal{A}_{T}=\int_{\mathcal{V}}|\nabla c| d V$. This surface is well defined and commonly used to compute the total flame surface in the flamelet regime. Its relevance in the TRZ regime is questionable but $\mathcal{A}_{T}$ is used in the present study as a reference allowing comparison of flames in the flamelet and TRZ regimes.

As expected, each case shows a transitory phase followed by a quasi steady-state with $\mathcal{A}_{T}$ oscillating around an average value. To verify that the flames are stationary, the temporal averaged rate of change of the flame surface $\left(1 / \mathcal{A}_{T}\right) \mathrm{d} \mathcal{A}_{T} / \mathrm{d} t$ was verified to be close to zero. Additionally, the temporal evolution of the mean flame brush thickness, $\delta_{T}$, is shown in Fig. 5. This thickness is approximated, as suggested by Lipatnikov and Chomiak [3], as $\delta_{T}=\max (|\nabla c|)^{-1}$, where $\widetilde{c}$ is the $\mathrm{y}-\mathrm{z}$ averaged progress variable at a given instant evaluated following the procedure in Appendix D. Figure 5 shows that each case presents a transitory phase followed by a quasi steady-state with $\delta_{T}$ oscillating around an average value. This observation is not consistent with experimental studies reported by Lipatnikov and Chomiak [3], where $\delta_{T}$ increased with time. However, Poludnenko and Oran [17, 70] observed in their DNS study, similar results as those in Fig. 5 when turbulence forcing is used.

From these observations, the quantities of interest extracted from DNS results are temporally averaged starting from the physical time when the coefficient of variation of the flame surface, which is the standard deviation divided by the mean value has reached $90 \%$ of its final value. Statistics are computed after the transient phase using at least 15 eddy turnover times $\tau_{t}=l_{t} / u^{\prime}$, to get sufficient convergence.

\subsection{Analysis of the turbulent flame velocity and wrin- kling}

From a macroscopic point of view the main effect of turbulence on the flame is to increase the propagation speed $S_{T}$ defined as:

$$
S_{T}=\frac{1}{\rho_{u} \mathcal{A}_{0}} \int_{\mathcal{V}} \dot{\omega}_{c} d V
$$




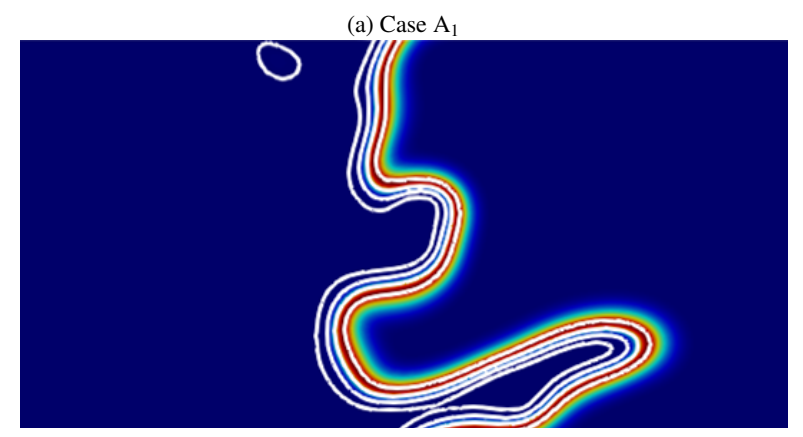

(b) Case $\mathrm{B}_{1}$

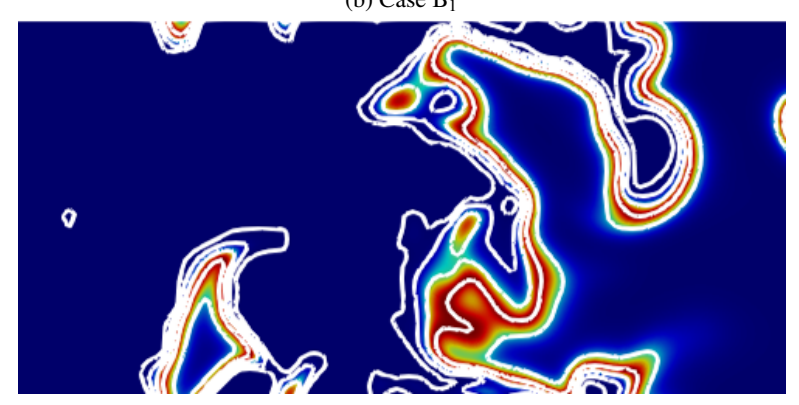

(c) Case $\mathrm{E}_{1}$

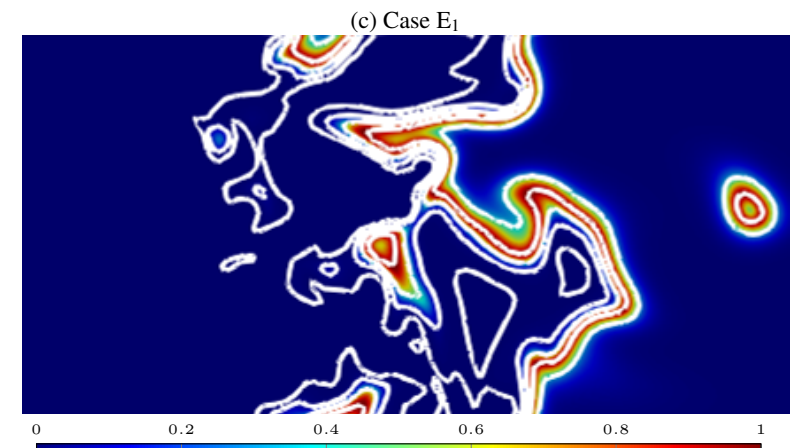

Figure 3: Two-dimensional slices in x-y mid-plane of the flames colored with the heat release rate normalized by its maximum value $\left(\dot{\omega}_{T}^{\prime} / \dot{\omega}_{T}^{\prime}\right.$ max $)$ and with contours of the progress variable from $c=0.1$ to $c=0.9$ (white lines), defined as the reduced temperature: (a) to for unity Lewis number cases, and (d) to ff for non-unity Lewis number cases.

where $\dot{\omega}_{c}=\dot{\omega}_{T}^{\prime} /\left(\left\langle C_{p}\right\rangle\left(T_{b}-T_{u}\right)\right)$ is the chemical source term with $\dot{\omega}_{T}^{\prime}$ the heat release rate, $\rho_{u}$ the density of the fresh gases, $\left\langle C_{p}\right\rangle$ is the mean heat capacity within the flame front and $\mathcal{A}_{0}$ the laminar flame surface corresponding to the y-z cross section of surface $L^{2}$. Damköhler attributed this acceleration to the increase in the flame front surface $\mathcal{A}_{T}$ by wrinkling, leading to:

$$
\frac{S_{T}}{S_{L}^{0}} \propto \frac{\mathcal{A}_{T}}{\mathcal{A}_{0}},
$$

The proportionality between the increase of the propagation speed and the flame wrinkling $\Xi=\mathcal{A}_{T} / \mathcal{A}_{0}$, is often quantified through the effect of the flame stretch

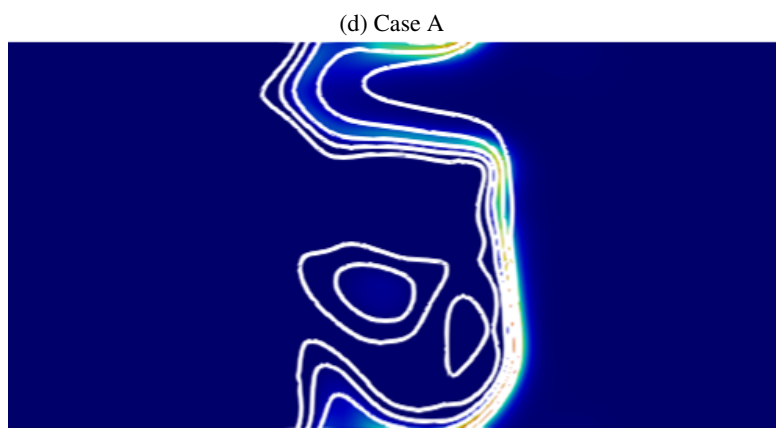

(e) Case B
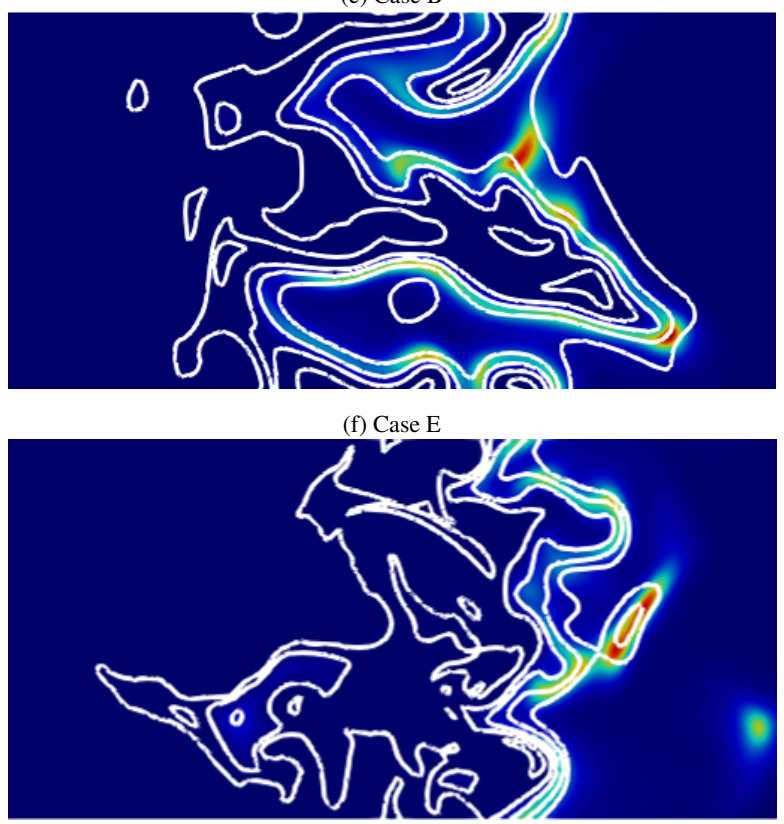

using the stretch factor defined by:

$$
I_{0}=\frac{S_{T}}{S_{L}^{0} \Xi}
$$

The evolution of the flame wrinkling (circles) and propagation speed (plain triangles) are plotted as functions of $K a$ in Figs. $6 \mathrm{~b}$ and $6 \mathrm{a}$, for $L e>1$ and $L e=1$ cases, respectively. For $L e>1$ cases, $\Xi$ and $S_{T}$ reach saturation simultaneously at a $K a$ corresponding to case $\mathrm{B}$, while for $L e=1$ cases the quantities even decrease between B and E. Such an effect of the Lewis number was for instance observed in the experiment of Yang et al. [4]. The maximum wrinkling and turbulent flame speed of $L e=1$ cases are also approximately 3.5 larger than that of $L e>1$ cases. This can be considered 

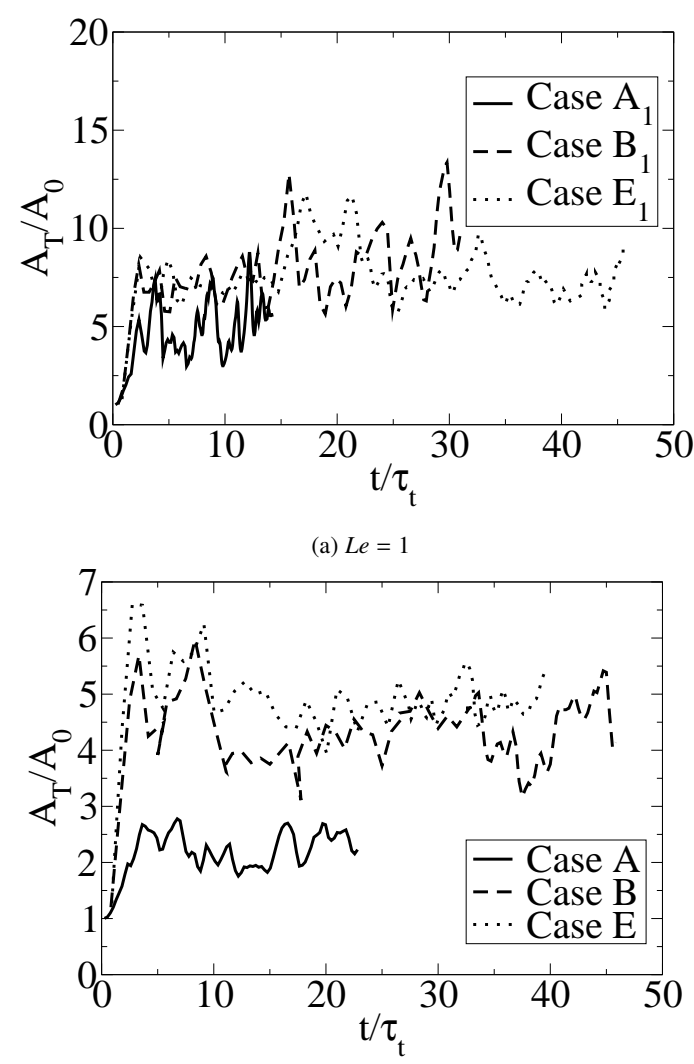

(b) $L e>1$

Figure 4: Temporal evolution of the turbulent flame surface $\mathcal{A}_{T}$ normalized with the laminar flame surface $\mathcal{A}_{0}$ a for cases with $L e=1$ and (b) for cases with $L e>1$.

the major impact of preferential diffusion on turbulent flames in the TRZ regime as mentioned in [3], and justifies the need to include preferential diffusion in the modeling of this regime, as shown by many previous experimental [12,-14] and DNS [42, 67] studies. Note that $S_{T}$ differs for $L e=1$ and $L e>1$ cases even for the flamelet cases. This might be due to the fact that case $\mathrm{A}$ is at the upper limit of flamelet regime, showing a Karlovitz number of 2.9. Such a value implies that the flame is subject to a stretch sufficiently intense to alter its displacement speed. Nevertheless, case A is still in the flamelet regime as its structure still corresponds to that of a disturbed laminar flame, as defined by Lipatnikov and Chomiak [3].

The evolution of the stretch factor $I_{0}$ with $K a$ is shown in Fig. 6c For unity Lewis number cases, $I_{0}$ remains close to unity, even at the largest $K a$, which corresponds to the proximity of $\Xi$ and $S_{T} / S_{L}$ in Fig. $6 a$ For non-unity Lewis cases, $I_{0}$ is already smaller than unity (0.7) for case A and decreases only slightly down to 0.6 for case E. These results indicate, in line with pre-

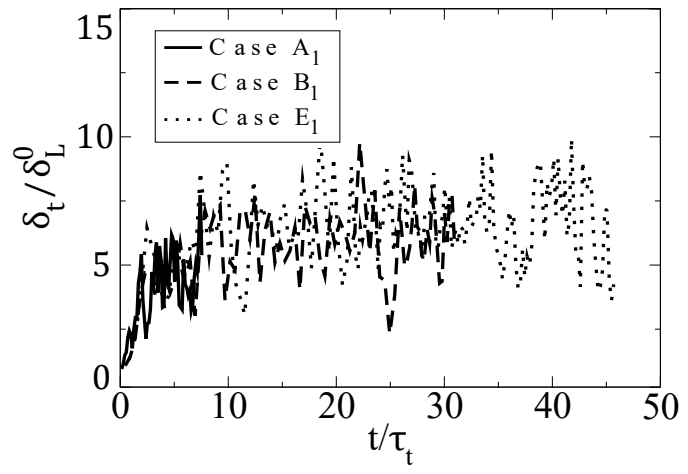

(a) $L e=1$

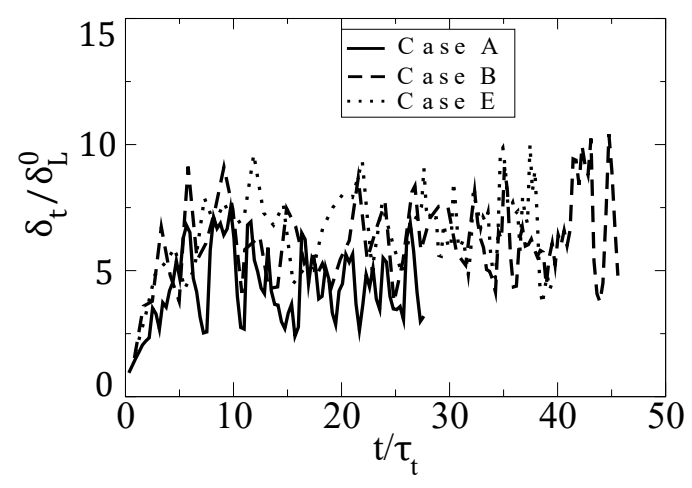

(b) $L e>1$

Figure 5: Temporal evolution of the mean flame brush thickness $\delta_{T}$ normalized with the laminar flame thickness $\delta_{L}$ a for cases with $L e=$ 1 and $(b)$ for cases with $L e>1$.

vious DNS [18, 40, 42, 66] that the local consumption speed of unity Lewis number flames is weakly affected by stretch, even in the TRZ regime, while flames showing preferential diffusion are affected in a larger extent. This difference of behavior is illustrated by the larger fluctuations of the heat release rate for $L e>1$ cases compared to $L e=1$ cases, as observed in Fig. 3. These fluctuations indicate that the local stretch rate strongly impacts the consumption speed. This aspect will be studied in the present paper considering the statistics of the displacement speed $S_{d}$. At the same time, although the average displacement (or consumption) speed is not that of the laminar flame, it still remains close to it. This result, in agreement with previous DNS [6, 11, 18] does not agree with recent analysis of experimental results [15] which conclude that this speed strongly increases in the TRZ regime. As discussed in the introduction, this topic requires further research.

The evolution of the wrinkling $\Xi^{*}$ on an iso-surface $c^{*}=0.8$, corresponding to the reaction zone, is also 

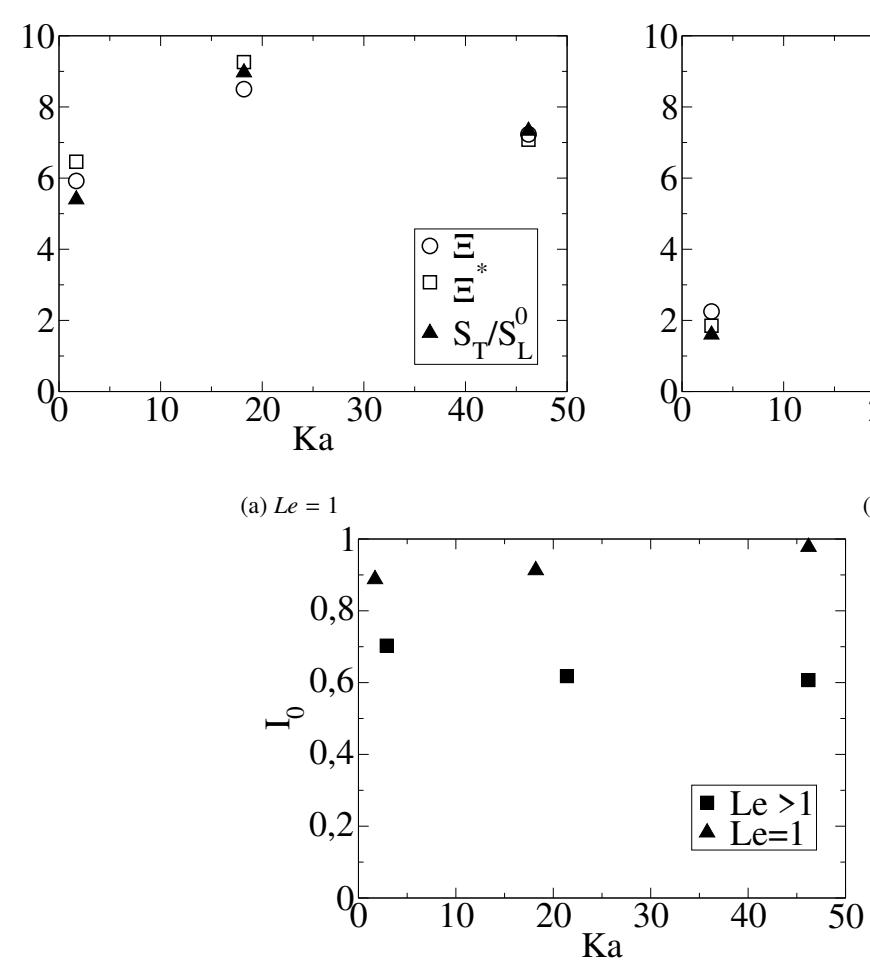

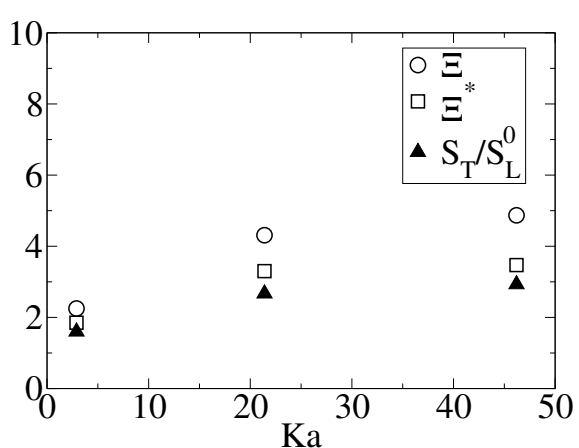

(b) $L e>1$

(c)

Figure 6: Evolutions of $\Xi, \Xi^{*}$ and $S_{T} / S_{L}^{0}$ with $K a$ using circle, square and triangle symbols, respectively, a for cases with $L e=1$ and b for cases with $L e>1$. (c) Evolutions of the stretch factor $I_{0}$ with $K a$ for $L e>1$ and $L e=1$ cases in triangle and diamond symbols, respectively.

plotted in Figs. 6b and 6a This wrinkling is defined as:

$$
\Xi^{*}=\frac{\mathcal{A}_{T}^{*}}{\mathcal{A}_{0}},
$$

where $\mathcal{A}_{T}^{*}=(1 / \tau) \int_{0}^{\tau} \int_{\mathcal{V}}|\nabla c(t, \mathbf{x})| \delta\left(c(t, \mathbf{x})-c^{*}\right) d \mathbf{x} d t$ is the area of the iso-surface $c^{*}=0.8$, with $\tau$ the period of the quasi-steady state phase retained for statistics computation. At the beginning of this phase, the time is set to 0 .

As expected from previous DNS [18, 40, 42, 66], when $L e=1$ and for case A, $\Xi^{*}$ and $\Xi$ are almost similar. This is in agreement with the flamelet assumption considering the flame infinitely thin, leading to a generalized flame surface $\mathcal{A}_{T}$ close to the area of any isosurface of progress variable. Figure $6 \mathrm{~b}$ shows a much better agreement of $\Xi^{*}$ with $S_{T}$ than $\Xi$ for high $K a$, which suggests that in the reaction zone the flamelet assumption is still valid.

\subsection{Analysis of the thickening of the flame front}

To quantify the apparent thickening of the flame, the thickening factor $\Theta$ introduced by Aspden et al. [53] is examined.

$$
\Theta(c)=\frac{\langle|\nabla c|\rangle_{c}^{r e f}}{\langle|\nabla c|\rangle_{c}},
$$

where $\langle|\nabla c|\rangle_{c}$ is the mean value of the progress variable gradient conditioned at $c$. In this study the normalization is made using the gradient of the laminar flame for both non-unity and unity Lewis number cases, while in [53] the normalization is performed using the conditional mean of the gradient for the case $K a=1$.

In Fig. 7a the evolution of $\Theta$ with the progress variable is shown for unity Lewis number cases. Case $\mathrm{A}_{1}$ shows an almost constant value equal to 1.5 , which indicates that the flame is uniformly thickened compared to the laminar flame. Cases $\mathrm{B}_{1}$ and $\mathrm{E}_{1}$ present a thickening on the fresh gases side $(c<0.5)$ up to a factor 2 compared to case $A_{1}$, but $\Theta$ converges to the value of case $A_{1}$ when $c>0.5$. This observation was already made by Aspden et al. in [53], where the thickening factor of a methane flame collapsed to that of a flame in the flamelet regime. So, when $K a$ increases, the flame is thickened on the fresh gases side but remains thin for $c>0.5$ for unity Lewis number cases as observed in experimental studies [71, 72]. 


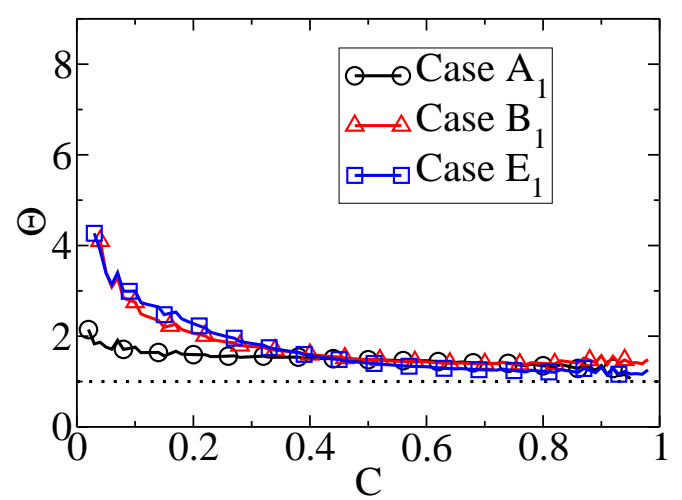

(a) $L e=1$

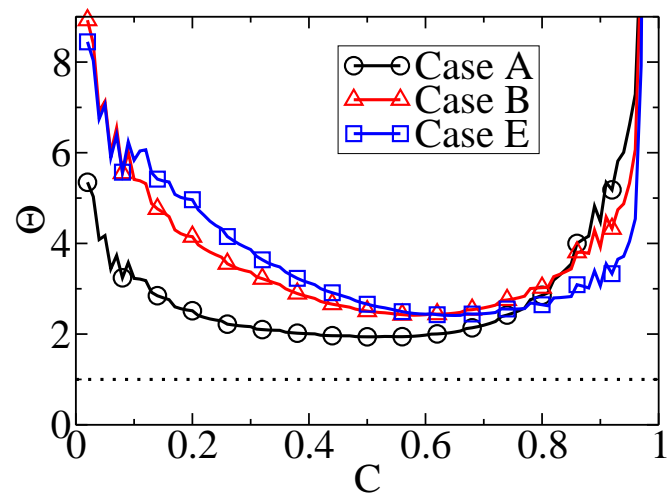

(b) $L e>1$

Figure 7: Thickening factor $\Theta(c)$ a for cases with $L e=1$ and $b$ for cases with $L e>1$.

The same quantity is shown for non-unity Lewis number simulations in Fig. $7 \mathrm{~b}$ For case A, $\Theta$ reaches values close to 5-6 for $c<0.3$ and $c>0.7$, but remains close to 2 elsewhere. The unexpected thickening observed on the fresh gases side might be due to the fact that case $\mathrm{A}$ is at the upper limit of the flamelet regime. Thus, the difference in thickness is attributed to the fact that even for case A, the flame is highly stretched, which will be shown in the next section through its displacement speed $S_{d}$ different from $S_{d}^{0}$. As a consequence, its thickness is not expected to correspond to that of a laminar unstretched flame. Compared to case A, cases $\mathrm{B}$ and $\mathrm{E}$ present an even larger thickening on the fresh and burnt gases sides, which is coherent with the observed non-parallel progress variable iso-surfaces in the preheat zone (Fig. 3). However, in the reaction zone $(0.5<c<0.9$ as seen in Fig. 8) the increase of the thickening compared to case A is relatively low, approximately 2.4 for case B and 2.6 for case E.

Figure 8 shows the conditional mean of the progress variable chemical source term $\dot{\omega}_{c}$, normalized by the

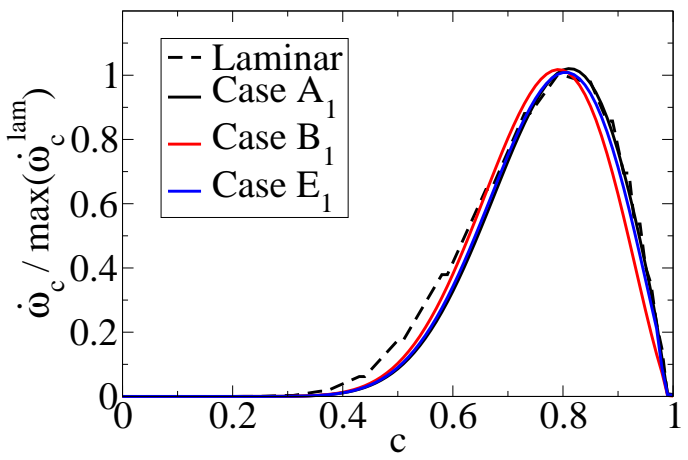

(a) $L e=1$

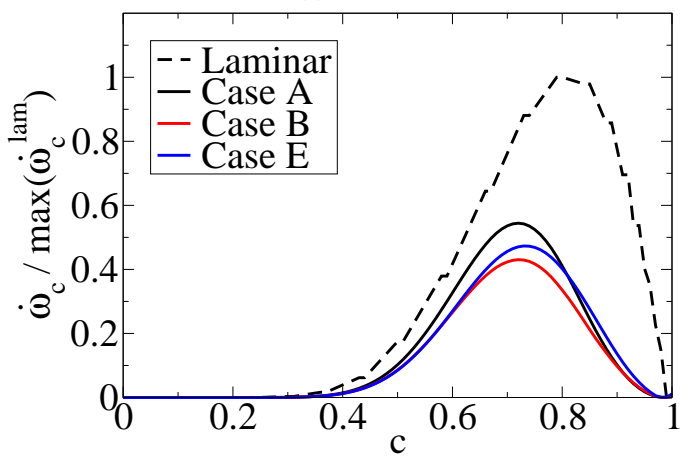

(b) $L e>1$

Figure 8: Conditional means of the chemical source term $\dot{\omega}_{c}(c)$, normalized by the peak chemical source term of the 1D laminar flame, as a function of progress variable: (a) for cases with $L e=1$, and (b) for $L e>1$ cases.

peak chemical source term of the 1D laminar flame, as a function of the progress variable $c$ for each case. As described in section 3 , the flames with unity Lewis number and non-unity Lewis number have the same $S_{L}^{0}$ and $\delta_{L}$. As a consequence, they present similar laminar chemical source term profiles as seen in Fig. 8. The profiles of cases $A_{1}, B_{1}$ and $E_{1}$ are very close to those of the 1D laminar flame, as observed in previous DNS studies. This suggests that with unity Lewis numbers, although the flame is slightly thickened, turbulence does not impact the inner flame structure significantly. It is noteworthy that, as turbulence intensity is increased, the fluctuations of the progress variable chemical source term are significantly enhanced, as observed in Fig. 3

On the contrary, the non-unity Lewis number cases show a decrease of the chemical source term when $\mathrm{Ka}$ increases, from a maximum of 0.55 for case A down to 0.43 for case B. However, when $\mathrm{Ka}$ increases further, the inverse tendency is observed with an increase of $\dot{\omega}_{c} / \max \left(\dot{\omega}_{c}^{\text {lam }}\right)$ up to 0.48 for case E. But the number of studied cases here is too low to provide a clear view on the evolution of this maximum as a function of $K a$. This phenomenon was also observed by Lapointe 
et al. [42], who suggested that as turbulent mixing is enhanced, differential diffusion effects are reduced. It can also be remarked that although peak values are slightly different for cases A, B and E, the chemical source term assumes very similar shapes for the three cases when $c>c_{\text {peak }}$, with $c_{\text {peak }} \approx 0.7$ corresponding to the maximum reaction rate. As observed by Lapointe et al. [42], $c_{\text {peak }}$ of the turbulent flames shifts from 0.8 to 0.7 for $L e>1$, which is due to preferential diffusion effects. This suggests that the inner structure of high Karlovitz flames B and E remains close to the structure of a flame in the flamelet regime (A) on the burnt gases side, in line with the definition of the TRZ regime. The broadening of the thickening factor on the burnt gases side, in Fig. 7b may be due to the normalization by the laminar case for which the temperature profile has a short tail (high gradients), resulting in higher $\Theta$ values. These observations are in good agreement with the two-dimensional fields of Fig. 3 .

The analysis proposed by Peters [1] considering a thin reaction zone for the TRZ regime is globally verified in the present DNS, but the impact of the Lewis number needs to be added: when $L e=1$ perturbations of the reaction zone can be neglected, while the flame is strongly thickened and the reactivity decreased for Le $>1$.

\section{4. modeling implications}

The progress variable source term corresponding to the RHS of Eq. (3) is the first quantity of interest for modeling. Its integral over the whole domain $\Omega_{t o t}$, is given by Eq. 23]:

$$
\Omega_{\text {tot }}=\int_{V} \rho S_{d}|\nabla c| d V=\left\langle\rho S_{d}\right\rangle_{s} \mathcal{A}_{T},
$$

where $\left\langle\rho S_{d}\right\rangle_{s}$ is the surface averaged displacement speed and $\mathcal{A}_{T}$ is the flame surface, both defined with the generalized approach of the CFM model.

Although $\Omega_{\text {tot }}$ is related to the flame displacement speed $S_{d}$, it equals the total progress variable reaction rate $\int_{\mathcal{V}} \dot{\omega}_{c} d V$ because the integral of the diffusive contribution $\nabla \cdot(\overline{\rho D \nabla c})$ to the displacement speed, Eq. 5 , sums to zero. This equality was verified in the present study.

As shown in previous sections, for $L e>1$ cases, the flame structure is very perturbed in the preheat zone while only moderately in the reaction zone. This suggests that modeling a particular $c$ iso-surface located on the reaction zone might be more relevant than considering all iso-surfaces. This is why we compare $\Omega_{\text {tot }}$ to the progress variable source term $\Omega_{c^{*}}$ conditioned on a $c^{*}$ iso-surface:

$$
\Omega_{c^{*}}=\int_{\mathcal{V}} \rho S_{d}|\nabla c| \delta\left(c-c^{*}\right) d V=\left\langle\left(\rho S_{d}\right)^{*}\right\rangle_{s} \mathcal{A}_{T}^{*} .
$$

In Eq. 24), $\delta(c)$ is the Dirac function, $\left\langle\left(\rho S_{d}\right)^{*}\right\rangle_{s}$ and $\mathcal{A}_{T}^{*}$ are the surface averaged density weighted displacement speed at $c=c^{*}$ and the area of the $c^{*}$ iso-surface, respectively. Note that $\Omega_{c^{*}}$ is not equal to the reaction rate on the iso-surface $c=c^{*}\left(\int_{V} \dot{\omega}_{c} \delta\left(c-c^{*}\right) d V\right)$.

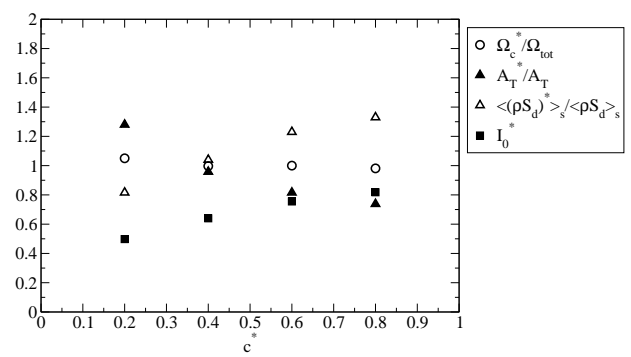

Figure 9: The evolution $\Omega_{c^{*}},\left\langle\rho S_{d}\right\rangle_{s}^{*}$ and $\mathcal{A}_{T}^{*}$ normalized by $\Omega_{t o t}$, $\left\langle\rho S_{d}\right\rangle_{s}$ and $\mathcal{A}_{T}$, respectively, as functions of the progress variable for case B.

Figure 9 presents the evolution of $\Omega_{c^{*}},\left\langle\left(\rho S_{d}\right)^{*}\right\rangle_{s}$ and $\mathcal{A}_{T}^{*}$ normalized by $\Omega_{t o t},\left\langle\rho S_{d}\right\rangle_{s}$ and $\mathcal{A}_{T}$, respectively, as functions of $c^{*}$ for case B. The stretch factor $I_{0}^{*}$ at $c^{*}$, defined using Eq. 25], is also plotted in Fig.9.

$$
I_{0}^{*}=\frac{\left\langle\left(\rho S_{d}\right)^{*}\right\rangle}{\rho_{u} S_{L}^{0}} .
$$

The first observation is that $\Omega_{c^{*}}$ is equal to $\Omega_{t o t}$ for all iso-surfaces. This result is in fact expected because the flame being statistically stationary, all iso-surfaces need to advance at the same velocity. On the other hand, $\mathcal{A}_{T}^{*}$ decreases substantially with increasing $c^{*}$. This result is consistent with Fig. 3 which shows a strong distortion of $c^{*}$ iso-surfaces towards $c^{*}=0$, corresponding to a strong increase of the flame surface $\mathcal{A}_{T}^{*}$. This illustrates once again that in the TRZ regime, $c^{*}$ iso-surfaces are not parallel to each other for low values of $c^{*}$. As shown by Eq. 24 larger values of $\mathcal{A}_{T}^{*}$ at small $c^{*}$ need to be compensated by smaller displacement speeds, i.e., by smaller values of $I_{0}^{*}$.

These results show that the modeling of $\mathcal{A}_{T}^{*}$ and $\left\langle\left(\rho S_{d}\right)^{*}\right\rangle_{s}$ will depend on the choice of $c^{*}$ in the TRZ regime. Figure 9 shows that $I_{0}^{*}$ increases significantly when $c^{*}$ increases from 0.2 to 0.8 and that it seems to reach a plateau, indicating again that in the reaction zone, progress variable iso-surfaces remains approximately parallel, unlike in the preheat zone. This is why in the rest of this study the flame will be studied through 
an iso-surface $c^{*}=0.8$, close to the maximum heat release rate iso-surface.

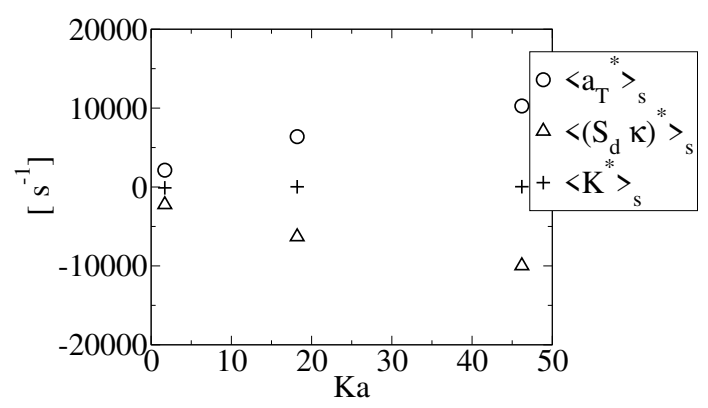

(a) $L e=1$

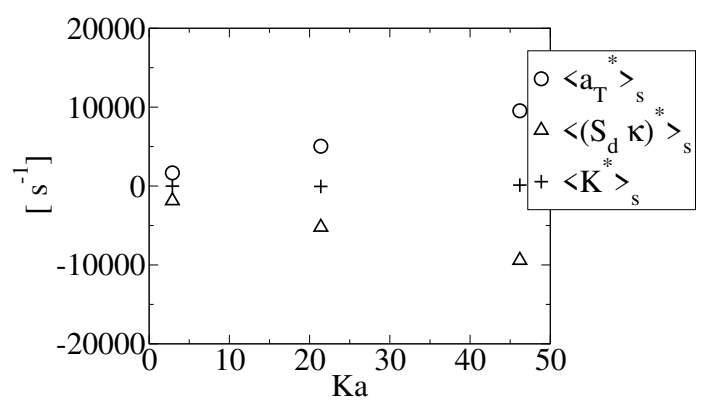

(b) $L e>1$

Figure 10: Evolutions of $\left\langle K^{*}\right\rangle_{s}=\left(1 / \mathcal{A}^{*}\right)\left(d \mathcal{A}^{*} / d t\right),\left\langle a_{T}^{*}\right\rangle_{s}$ and $\left\langle\left(S_{d} \kappa\right)^{*}\right\rangle_{s}$ with $K a$ using plus, circle and triangle symbols, respectively: (a) for cases with $L e=1$ and (b) for cases with $L e>1$.

To check the equilibrium state on this particular isosurface, the flame stretch balance at $c^{*}=0.8$ is shown in Fig. 10. For each simulation the rate of change of $\mathcal{A}^{*}$ is close to zero. This figure also shows that the source terms of flame surface, the tangential strain rate $\left\langle a_{T}^{*}\right\rangle_{s}$, and the stretch due to curvature $\left\langle\left(S_{d} \kappa\right)^{*}\right\rangle_{s}$ compensate exactly, and show a quasi linear increase with $K a$.

As seen in Fig. 6 the values of the wrinkling $\Xi^{*}$ are smaller than $\Xi$ and closer to $S_{T} / S_{L}^{0}$. This is coherent with the fact that $I_{0}^{*}$ is closer to unity than $I_{0}$, and justifies the choice of the iso-surface $c^{*}=0.8$ for modelling, rather than the generalized flame surface.

\subsection{Analysis of the displacement speed on an iso- surface}

A more thorough analysis of the displacement speed is now presented with a priori modeling as an objective. In the following, the exponent $*$ refers to the value of variables on the iso-surface $c^{*}=0.8$.

Fig. 11 presents the mean displacement speed $\left\langle S_{d}^{*}\right\rangle_{\kappa^{*}}$

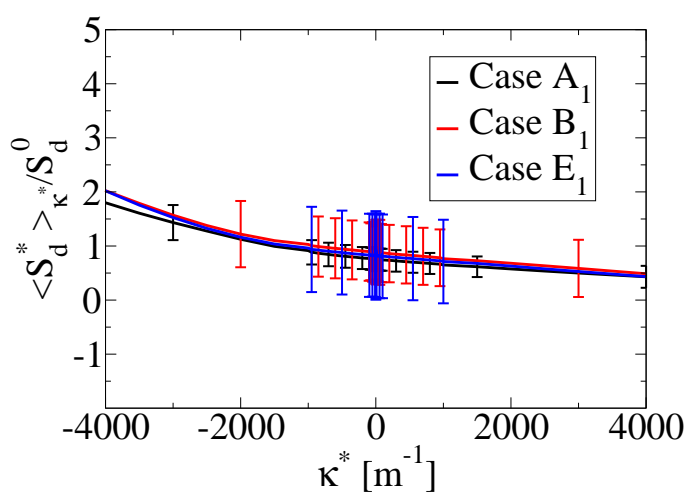

(a) $L e=1$

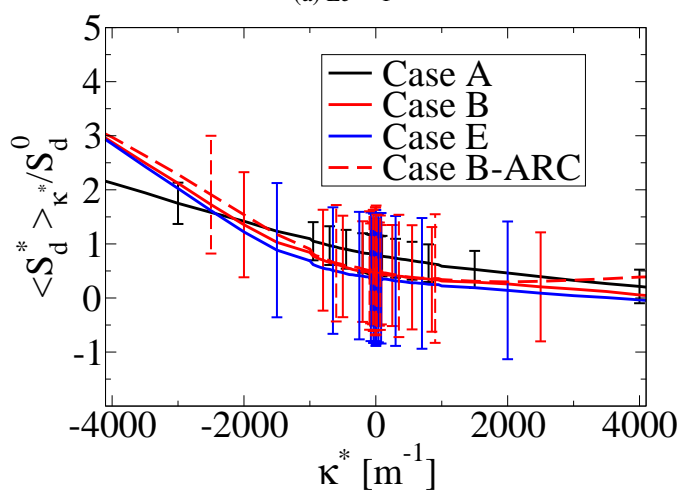

(b) $L e>1$

Figure 11: Evolution of the conditional mean of the displacement speed $\left\langle S_{d}^{*}\right\rangle_{\kappa^{*}}$, normalized by the laminar flame speed $S_{d}^{0}$ at $c=c^{*}$, with curvature $\kappa^{*}$ : (a) for cases with $L e=1$ and (b) for cases with $L e>1$. The error bars represent the standard deviation of $S_{d}^{*}$ from the DNS.

conditioned on $\kappa^{*}$, the flame curvature. The displacement speed is normalized with $S_{d}^{0}=\rho_{u} S_{L}^{0} / \rho^{*}$, the displacement speed of the planar laminar flame at $c=c^{*}$. The standard deviation of $S_{d}^{*}$ from the DNS is also reported by error bars. As observed in previous studies for $L e>1$ [7, 30, 34, 35, 73], Fig. 11b shows a negative correlation between displacement speed and curvature. It also shows an increase of the slope with the Karlovitz number. Case B-ARC performed using an ARC mechanism is seen to be very close to case $B$ performed with the 2-step mechanism. This indicates that the influence of curvature on the displacement speed is hardly sensitive to the level of chemistry description. In Fig. 11a the same results are presented for $L e=1$ cases: the negative correlation is also observed but the slope is smaller, and is marginally affected by the increase of the turbulence intensity.

To further analyze the displacement speed, the conditional statistics of the different contributions to $S_{d}^{*}$, as defined in Eqs. (10) to 12, are plotted as a function 


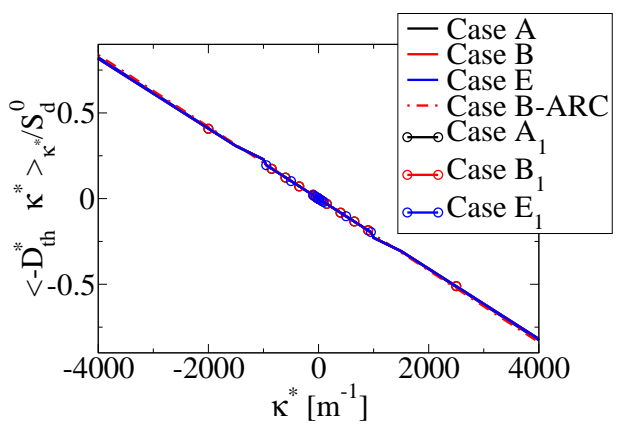

(a)

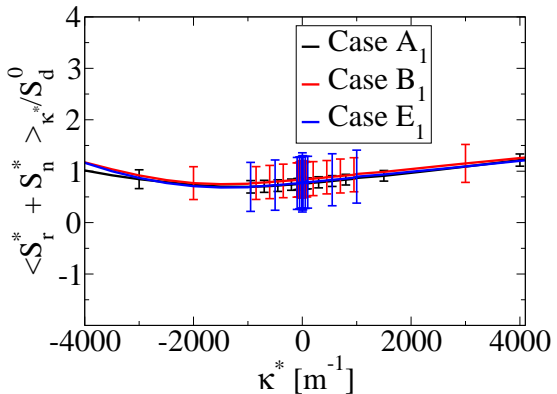

(b) $L e=1$

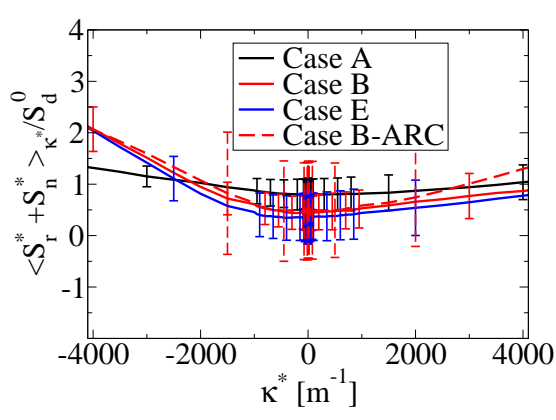

(c) $L e>1$

Figure 12: Evolution of the conditional mean of the components of the displacement speed, normalized with the laminar flame speed $S_{d}^{0}$ at $c=c^{*}$, with curvature $\kappa^{*}$ : a) $\left\langle-D_{t h}^{*} \kappa_{\kappa^{*}}\right.$ for all the cases, (b) $\left\langle S_{r}^{*}+S_{n}^{*}\right\rangle_{\kappa^{*}}$ for cases with $L e=1$ and c) $\left\langle S_{r}^{*}+S_{n}^{*}\right\rangle_{k^{*}}$ for cases with $L e>1$. The error bars display the standard deviation of $S_{r}^{*}+S_{n}^{*}$ from the DNS.

of curvature in Fig. 12 Fig. $12 \mathrm{a}$ shows the tangential diffusion $\left\langle S_{t}^{*}\right\rangle_{\kappa^{*}}=-D^{*} \kappa^{*}$. The thermal diffusivity $D^{*}$ depends on the mixture composition and temperature but is assumed to be the same for $L e=1$ and $L e>1$ flames. With this reasonable hypothesis, differences observed on $\left\langle S_{d}^{*}\right\rangle_{\kappa^{*}}$ are entirely due to the sum of $\left\langle S_{r}^{*}\right\rangle_{\kappa^{*}}$ and $\left\langle S_{n}^{*}\right\rangle_{\kappa^{*}}$.

Figs. $12 \mathrm{c}$ and $12 \mathrm{~b}$ present the sum $\left\langle S_{r}^{*}+S_{n}^{*}\right\rangle_{\kappa^{*}}$ as a function of curvature for the same flames. The evolution of $\left\langle S_{r}^{*}+S_{n}^{*}\right\rangle_{\kappa^{*}}$ differs from $\left\langle S_{d}^{*}\right\rangle_{\kappa^{*}}$ mainly for positive curvature where the correlation is positive between $\left\langle S_{r}^{*}+S_{n}^{*}\right\rangle_{\kappa^{*}}$ and $\kappa^{*}$. However, it can be observed that as for $\left\langle S_{d}^{*}\right\rangle_{\kappa^{*}}$, non-unity Lewis number flames show increasing slopes for increasing $K a$, while $L e=1$ flames show weak sensitivity to $K a$.

At $\kappa^{*}=0$, we could expect $\left\langle S_{d}^{*}\right\rangle_{\kappa^{*}} / S_{d}^{0}$ to be close to unity, which is not the case: for $L e>1$, it ranges between 0.37 and 0.8 , while for $L e=1$ flames, it takes larger values close to 0.85 . Figure 13 shows that the mean tangential strain rate conditioned with curvature, $\left\langle a_{T}^{*}\right\rangle_{\kappa^{*}}$, is not zero at $\kappa^{*}=0$ meaning that the flame is positively strained. Moreover, the standard deviation of $a_{T}^{*}$ from the DNS reported as error bars in Fig. 13 are very large, particularly for cases $\mathrm{E}$ and $\mathrm{E}_{1}$, and might also be a reason for $\left\langle S_{d}^{*}\right\rangle_{\kappa^{*}}$ to be far from $S_{d}^{0}$ at $\kappa^{*}=0$.
This dependency of $\left\langle S_{r}^{*}+S_{n}^{*}\right\rangle_{\kappa^{*}}$ to turbulence intensity is in agreement with previous studies [30, 34, 37, 73]. Figure 12 suggests that analyzing the response of the curvature alone is not sufficient and a double conditioning on both curvature and strain is required, as presented in Fig. 14. The joint probability density function of curvature and strain rate is also plotted in this figure by black solid lines.

The correlation of $a_{T}^{*}$ and $\kappa^{*}$ is reported in Table 3. It is evaluated using Pearson's correlation coefficient [74], defined as:

$$
r_{X, Y}=\frac{\sum_{i=1}^{n}\left(X_{i}-\langle X\rangle\right)\left(Y_{i}-\langle Y\rangle\right)}{\sqrt{\left(X_{i}-\langle X\rangle\right)^{2}} \sqrt{\left(Y_{i}-\langle Y\rangle\right)^{2}}},
$$

where $\left(X_{i}, Y_{i}\right)$ is a sample pair of data, \langle\rangle denotes the sample mean, and $n$ is the sample size. As already ob-

Table 3: Pearson's correlation coefficient, $r_{a_{T}^{*}, \kappa^{*}}$, between strain rate $a_{T}^{*}$ and curvature $\kappa^{*}$ for the flames presented in this paper.

\begin{tabular}{lcccccc}
\hline Case & $\mathrm{A}$ & $\mathrm{B}$ & $\mathrm{E}$ & $\mathrm{A}_{1}$ & $\mathrm{~B}_{1}$ & $\mathrm{E}_{1}$ \\
\hline$K a$ & 2.9 & 21.4 & 46.2 & 2.7 & 18.5 & 46.2 \\
$r_{a_{T}^{*}, \kappa^{*}}$ & -0.75 & -0.42 & -0.23 & -0.61 & -0.43 & -0.32 \\
\hline
\end{tabular}

served in the literature [75], the correlation of $a_{T}^{*}$ and 


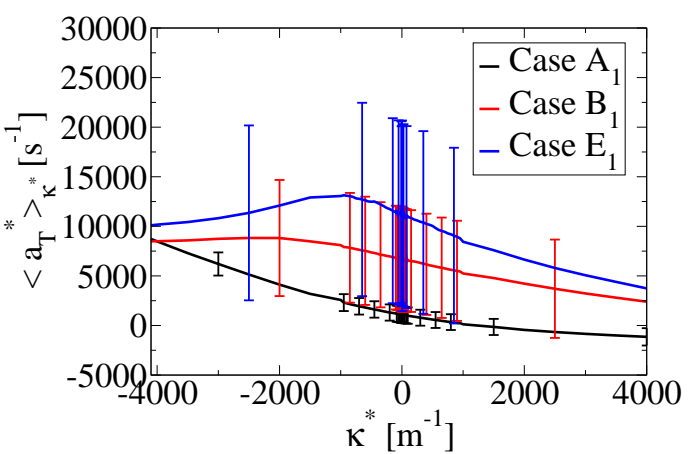

(a) $L e=1$

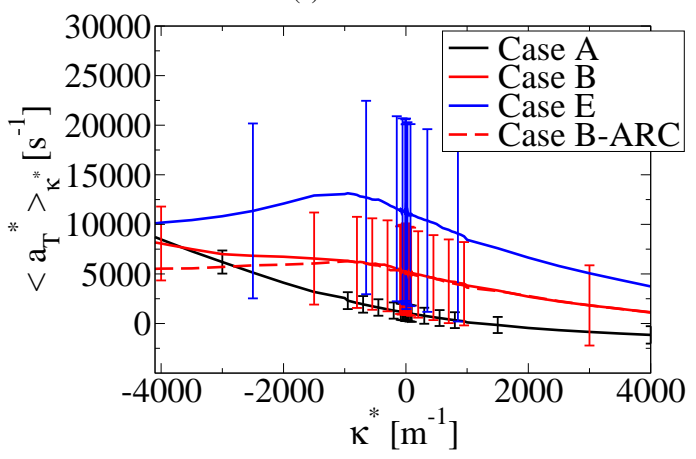

(b) $L e>1$

Figure 13: Evolution of the conditional mean of tangential strain $\left\langle a_{T}^{*}\right\rangle_{\kappa^{*}}$ with curvature $\kappa^{*}:$ a for cases with $L e=1$ and (b) for cases with $L e>1$. The error bars display the standard deviation of $a_{T}^{*}$ from the DNS.

$\kappa^{*}$ is negative and decreases as $K a$ increases. At low $K a$, the correlation is still strong for case $\mathrm{A}$ and already weaker for case $A_{1}$. As the Karlovitz number increases, the correlation decreases similarly for $L e=1$ and $L e>1$ flames.

Figure 14 illustrates the complexity of the dependency of the displacement speed with strain and curvature. The evolution of $\left\langle S_{r}^{*}+S_{n}^{*}\right\rangle_{\left(a_{T}^{*}, \kappa^{*}\right)}$ with $\kappa^{*}$ (not displayed for the sake of brevity) presents the same trend as the evolution of the conditional mean $\left\langle S_{r}^{*}+S_{n}^{*}\right\rangle_{\kappa^{*}}$ in Figs. $12 \mathrm{c}$ and $12 \mathrm{~b}$ but the slopes depend on the value of $a_{T}^{*}$. The evolution of $\left\langle S_{r}^{*}+S_{n}^{*}\right\rangle_{\left(a_{T}^{*}, \kappa^{*}\right)}$ with $a_{T}^{*}$ seems linear with a slope that depends on $K a$ and differential diffusion (through the Lewis number). Thus, when the flame is stretched, the displacement speed responds differently to tangential strain and curvature.

Figure 14 shows that a model for $S_{d}^{*}$ needs to account for both strain rate $a_{T}^{*}$ and curvature $\kappa^{*}$. An expression of $S_{d}^{*}$ accounting for both strain and curvature has been proposed for a long time for laminar flames [76], and was more recently adapted for turbulent flames by Dave and Chaudhuri [27]. We propose an expression for $S_{d}^{*}$ adapted from the work of Peters [1] for the TRZ regime. Peters [1] modelled the laminar flame speed as $S_{L}=S_{L}^{*}-D \kappa$, where D is the diffusion coefficient of the deficient species. Peters [1] argues that $S_{L}^{*}$, which corresponds to $S_{r}^{*}+S_{n}^{*}$, can be approximated as $S_{d}^{0}$ in a first step, but should account for curvature, while $D$ should also depend on curvature. The observations of Fig. 14 lead to the conclusion that specific Markstein lengths for strain rate and curvature should be introduced like in laminar flame expressions for $S_{r}^{*}+S_{n}^{*}$. Although there is no theoretical justification for that, previous studies $[31-33,40,-42]$ developed the idea that the flame structure under strong turbulent conditions might be represented in average as that of a laminar flame with modified diffusivities and Markstein lengths. Following this idea, the following expression for $S_{d}^{*}=S_{r}^{*}+S_{n}^{*}-D^{*} \kappa^{*}$ is retained:

$$
S_{d}^{*}=S_{d}^{0}-\mathcal{L}_{a_{T}}^{t} a_{T}^{*}-\mathcal{L}_{\kappa}^{t}\left(S_{d} \kappa\right)^{*}-D^{*} \kappa^{*},
$$

where $\mathcal{L}_{a_{T}}^{t}$ and $\mathcal{L}_{\kappa}^{t}$ are turbulent Markstein lengths for strain and curvature that need to be defined. Note that the Markstein lengths used in Eq. (27) play the same role as the Markstein lengths used in the theory of perturbed laminar flames, but they are evidently not obtained in the limit of asymptotically small strain rate or curvature like laminar values. In particular, while in laminar asymptotic theories, this limit is reached by increasing the perturbation length scale, this length scale is here dictated by turbulence and can therefore be of the order or even smaller than the laminar flame thickness. To avoid confusion, we note these lengths $\mathcal{L}_{a_{T}}^{t}$ and $\mathcal{L}_{\kappa}^{t}$, whereas laminar values are noted $\mathcal{L}_{a_{T}}^{l}$ and $\mathcal{L}_{\kappa}^{l}$.

Dave and Chaudhuri [27] used a similar model for $S_{d}$ (Eq. (1.6) in their paper) based on the one proposed by Giannakopoulos et al. [28] for laminar flames. Expression (27) differs from that of Dave and Chaudhuri [27] because the influence of strain rate and curvature is accounted for on $S_{r}+S_{n}$ and not on $S_{d}$, which also means the term $-D \kappa$ is retained. Besides, while in [27], laminar Markstein lengths are conserved, they are here shown to be strongly dependent on the Karlovitz number. This difference might be explained by the larger Karlovitz considered in this study.

Equation (27) is implicit as the stretch due to curvature is based on $\left(S_{d} \kappa\right)^{*}$. It can be rearranged in an explicit form as:

$$
S_{d}^{*}=\frac{S_{d}^{0}-\mathcal{L}_{a_{T}}^{t} a_{T}^{*}-D^{*} \kappa^{*}}{1+\mathcal{L}_{\kappa}^{t} \kappa^{*}}
$$

In this study, values for $\left(\mathcal{L}_{a_{T}}^{t}, \mathcal{L}_{\kappa}^{t}\right)$ are first determined from a least square method to best fit the results of 
(a) Case $\mathrm{A}_{1}$

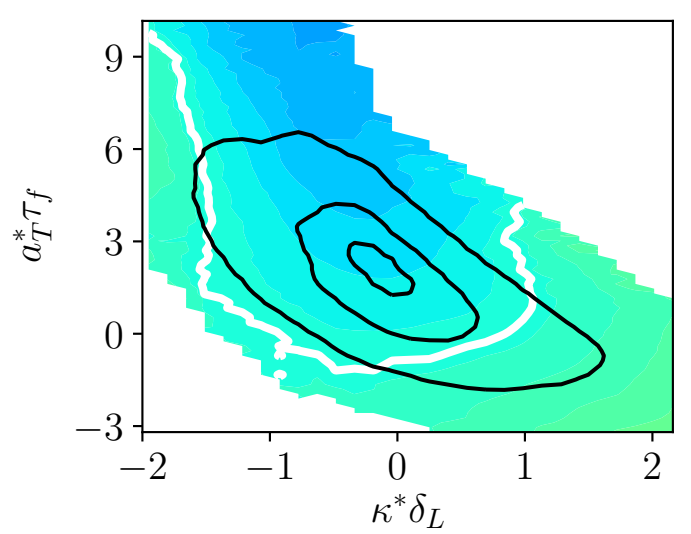

(b) Case $\mathrm{B}_{1}$

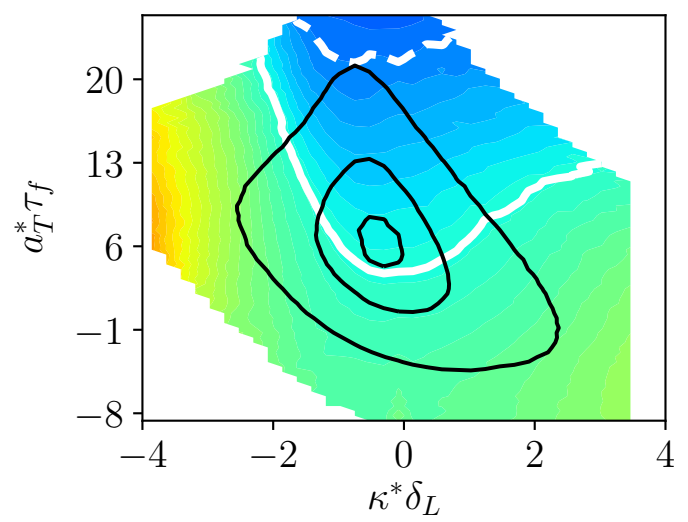

(c) Case $E_{1}$

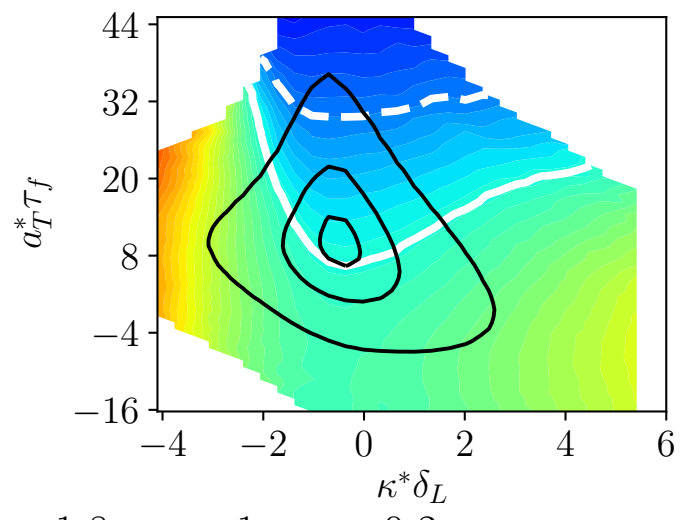

(d) Case A

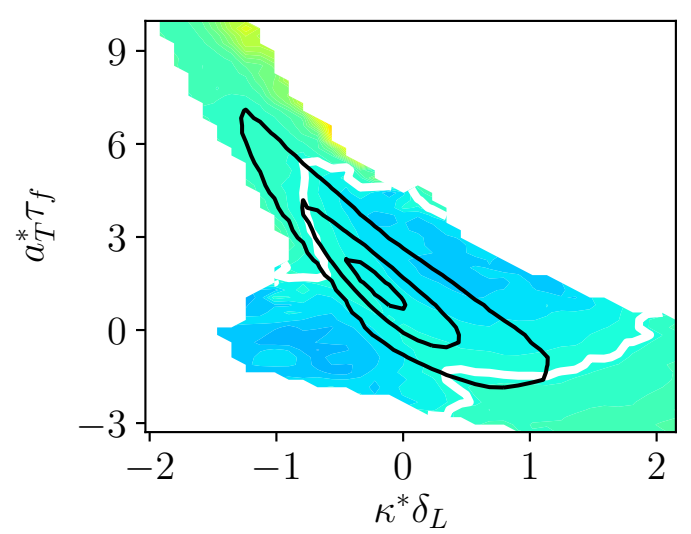

(e) Case B

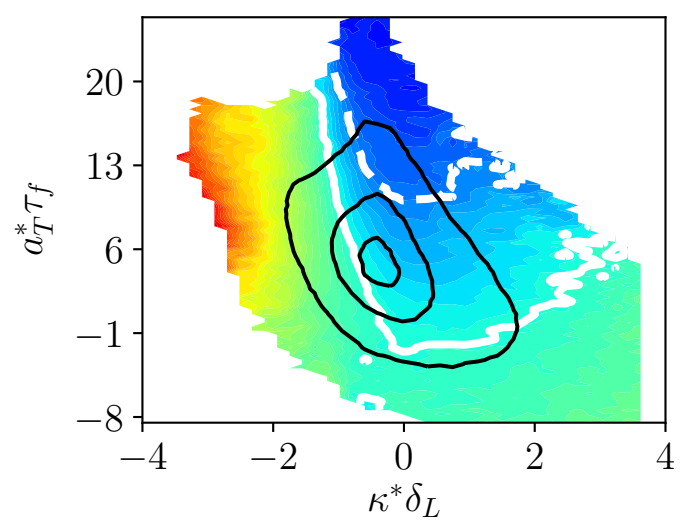

(f) Case E

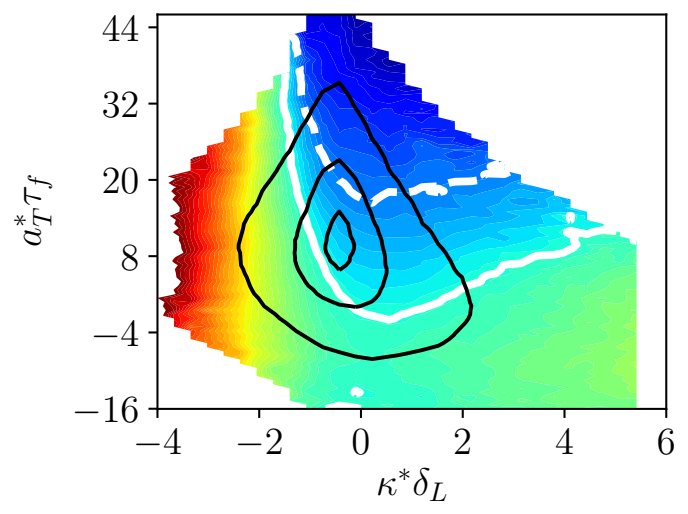

$\begin{array}{lllll}2.2 & 3 & 3.8 & 4.6 & 5.4\end{array}$

$$
\left\langle S_{r}^{*}+S_{n}^{*}\right\rangle_{\left(a_{T}^{*}, \kappa^{*}\right)} / S_{d}^{0}
$$

Figure 14: Evolution of the conditional mean of $\left\langle S_{r}^{*}+S_{n}^{*}\right\rangle_{\left(a_{T}^{*}, \kappa^{*}\right)}$ with $a_{T}^{*}$ and $\kappa^{*}$ normalized respectively by the flame time-scale and the laminar flame thickness for: a to [c] the cases with $L e=1$ and (d) to ff the cases with $L e>1$. Black lines are contours of the joint probability density function (PDF) of $a_{T}^{*}$ and $\kappa^{*}$. The solid white line is the iso-contour $\left\langle S_{r}^{*}+S_{n}^{*}\right\rangle_{\left(a_{T}^{*}, \kappa^{*}\right)}=\rho_{u} S_{L} / \rho^{*}$ and the dashed white line is the iso-contour $\left\langle S_{r}^{*}+S_{n}^{*}\right\rangle_{\left(a_{T}^{*}, \kappa^{*}\right)}=0$. 


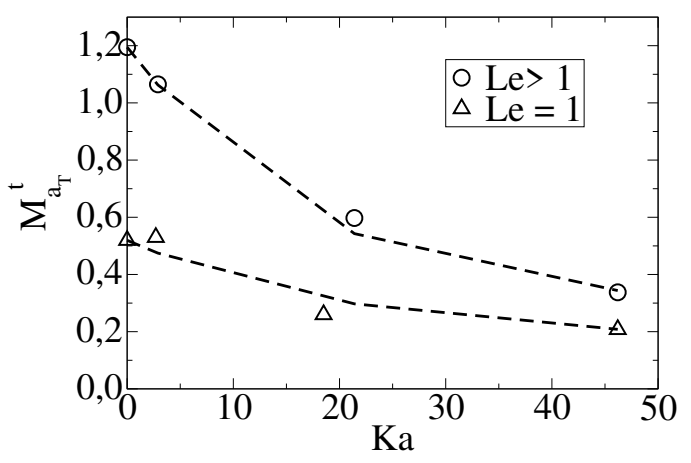

(a)

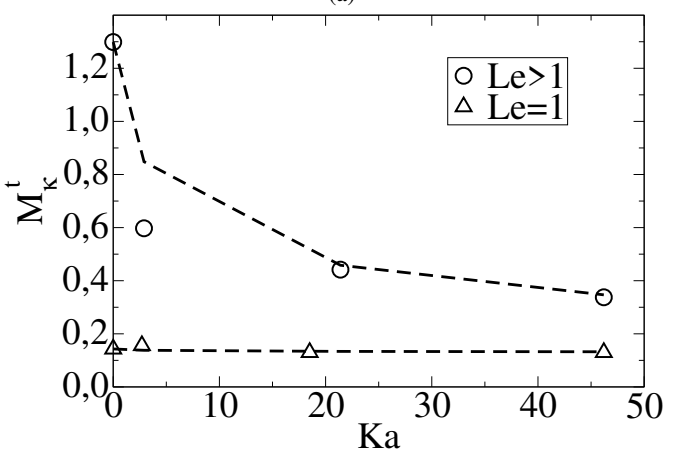

(b)

Figure 15: Evolution of effective Markstein numbers with $\mathrm{Ka}$ : a for $\mathcal{M}_{a_{T}}^{t}=\mathcal{L}_{a_{T}}^{t} / \delta_{L}$ and $\mathrm{b}$ for $\mathcal{M}_{\kappa}^{t}=\mathcal{L}_{\kappa}^{t} / \delta_{L}$. The values computed from stretched laminar flame and from the optimization methods are shown with markers and the dashed lines represent the models in Eqs. 30. and 31 .

Fig. 14 for each case. In addition, the difference $\Delta_{S}$ between the DNS and the model values given by Eq. (27) is weighted by the joint PDF of $a_{T}^{*}$ and $\kappa^{*}$ following Eq. (29) to avoid edge effects and to give more importance to the most probable strain and curvature values:

$\Delta_{S}=p\left(a_{T}^{*}, \kappa^{*}\right)\left(\left\langle S_{r}^{*}+S_{n}^{*}\right\rangle_{\left(a_{T}^{*}, \kappa^{*}\right)}-\left[S_{d}^{0}-\mathcal{L}_{a_{T}}^{t} a_{T}^{*}-\mathcal{L}_{\kappa}^{t}\left(S_{d} \kappa\right)^{*}\right]\right)$,

where $p\left(a_{T}^{*}, \kappa^{*}\right)$ is the joint PDF of $a_{T}^{*}$ and $\kappa^{*}$.

The evolution of $\mathcal{M}_{a_{T}}^{t}=\mathcal{L}_{a_{T}}^{t} / \delta_{L}$ and $\mathcal{M}_{\kappa}^{t}=\mathcal{L}_{\kappa}^{t} / \delta_{L}$ with turbulent intensity are plotted in Fig. 15 for all cases. The values of $\mathcal{L}_{a_{T}}^{l}$ and $\mathcal{L}_{K}^{l}$ for $K a=0$ are obtained using stretched laminar flames simulations (detailed in Appendix B): a counter-flow premixed flame to evaluate $\mathcal{L}_{a_{T}}^{l}$ using the open-source solver Cantera, and a spherical flame for $\mathcal{L}_{\kappa}^{l}$ using the AVBP code.

For $L e>1$ flames, it can be observed that $\mathcal{M}_{a_{T}}^{t}$ and $\mathcal{M}_{\kappa}^{t}$ decrease with the Karlovitz number as expected from previous studies [18, 39, 43, 77, -79], and that their value becomes much smaller than their laminar value (approximately one third at $\mathrm{Ka}=46$ ). Savard et al.
[18, 39- 43] suggest that when turbulence is sufficiently intense, the turbulent diffusivity $D_{T}$ becomes dominant compared to thermal or mass diffusivities. This leads them to define an effective Lewis number which tends toward unity when $\mathrm{Ka}$ increases. In this study, a similar effect is observed through the effective Markstein numbers which tend toward zero when $\mathrm{Ka}$ increases. The values obtained for case A are also seen to be already quite smaller than the laminar ones (at $K a=0$ ), particularly for curvature. This might indicate that the curvature effect on the flame displacement speed decreases more rapidly than the strain effect when $\mathrm{Ka}$ increases.

Concerning the effect of strain, Fig. 15 shows that $L e=1$ and $L e>1$ cases present a similar decrease of $\mathcal{L}_{a_{T}}^{t}$ with $K a$. For laminar $(K a=0)$ and turbulent conditions, $\mathcal{L}_{a_{T}}^{t}$ for $L e=1$ flames is approximately half the value of $L e>1$ cases. This indicates that although thermo-diffusive effects are absent at $L e=1$, the effect of strain rate on the local displacement speed cannot be neglected. Concerning curvature, $\mathcal{L}_{K}^{t}$ for $L e=1$ cases shows an almost constant value close to the laminar one, which indicates the sensitivity to curvature is not altered by turbulence when differential diffusion is absent. For $L e>1$ cases, $\mathcal{L}_{\kappa}^{t}$ shows on the contrary a decrease with $K a$ qualitatively similar to the one observed for $\mathcal{L}_{a_{T}}^{t}$. These observations might be specific to the present fuel, thermodynamic conditions and chemical mechanism and cannot be generalized to all unity and larger than unity Lewis number flames. It is shown in Appendix C that the prediction of strain effect on laminar premixed flames depends quantitatively on the choice of the chemical mechanism but the response of turbulent flames to strain rate only slightly differs between the mechanisms. However a similar evolution was described by Savard and Blanquart [39] for the effective Lewis number they introduced. Using DNS of , lean premixed turbulent hydrogen flames they observed that the flame tends to behave like a unity Lewis number flame when the turbulence intensity increases sufficiently. From this observation, an expression for the effective Lewis number was proposed, the form of which is here retained for both $\mathcal{L}_{a_{T}}^{t}$ and $\mathcal{L}_{\kappa}^{t}$ :

$$
\begin{aligned}
\frac{\mathcal{L}_{a_{T}}^{t}}{\delta_{L}} & =\frac{1}{1+\alpha K a^{a}} \frac{\mathcal{L}_{a_{T}}^{l}}{\delta_{L}}+\left(1-\frac{1}{1+\alpha K a^{a}}\right) \mathcal{M}_{a_{T}}^{\infty}, \\
\frac{\mathcal{L}_{K}^{t}}{\delta_{L}} & =\frac{1}{1+\beta K a^{b}} \frac{\mathcal{L}_{\kappa}^{l}}{\delta_{L}}+\left(1-\frac{1}{1+\beta K a^{b}}\right) \mathcal{M}_{\kappa}^{\infty},
\end{aligned}
$$

where $\mathcal{L}_{a_{T}}^{l}, \mathcal{L}_{\kappa}^{l}$ are the laminar values while $\mathcal{M}_{a_{T}}^{\infty}$ and $\mathcal{M}_{\kappa}^{\infty}$ are asymptotic Markstein numbers at the upper range of the TRZ regime, and $\alpha, \beta, a$ and $b$ are model 
parameters. These parameters, reported in Table 4 are chosen using a least square method to best fit the evolution of $\mathcal{L}_{a_{T}}^{t}$ and $\mathcal{L}_{\kappa}^{t}$. Note that $\mathcal{M}_{a_{T}}^{\infty}$ and $\mathcal{M}_{\kappa}^{\infty}$ take small values in the present DNS. It is important to note that the proposed correlations for the effective Markstein lengths don't pretend to be universal. First, their mathematical form is only intended to reproduce the present DNS and is not based on physical arguments, excepted for the exponent $a$ which should be of the order of unity following the arguments of Savard and Blanquart [39]. Secondly, the present DNS results assess the influence of $K a$ with only three Karlovitz values, for given mixture and thermodynamic conditions. It is thus possible that other parameters would affect these effective Markstein lengths. In addition, Markstein lengths are known to be strongly dependent on the chosen iso-surface [3], making Eqs. (30) and (31) only meaningful for the isosurface $c^{*}=0.8$. All these remarks indicate that future research is needed on this topic.

As observed in Fig. 15, the proposed correlation recovers qualitatively the evolution of $\mathcal{L}_{a_{T}}^{t}$ and $\mathcal{L}_{\kappa}^{t}$ with $K a$, although at low $K a$ the error is quite important for $\mathcal{L}_{\kappa}^{t}$.

Table 4: Fitted parameters for modeling the Markstein numbers with Eqs. 30, and 31.

\begin{tabular}{cccccc}
\hline$\alpha$ & $a$ & $\beta$ & $b$ & $\mathcal{M}_{a_{T}}^{\infty}$ & $\mathcal{M}_{\kappa}^{\infty}$ \\
\hline 0.04 & 1.2 & 0.3 & 0.7 & 0.08 & 0.14 \\
\hline
\end{tabular}

The behavior of Eq. 27p is assessed on $\left\langle S_{r}^{*}+S_{n}^{*}\right\rangle_{\left(a_{T}^{*}, \kappa^{*}\right)}$, using the correlations in Eqs. 30 and (31) for the turbulent Markstein lengths, for each case in Figs. 16 and 17.

Figure 16 shows the evolution of $\left\langle S_{r}^{*}+S_{n}^{*}\right\rangle_{\left(a_{T}^{*}, \kappa^{*}\right)}$ with $a_{T}^{*}$ for the DNS and Eq. 27), considering a fixed $\kappa^{*}$ corresponding to the maximum of the joint PDF of $a_{T}^{*}$ and $\kappa^{*}$. In the same figure is plotted the PDF of $a_{T}^{*}$ when $\kappa^{*}$ is fixed. For $L e=1$ cases, Eq. 27) allows to predict properly the DNS results in Figs. 16a to 16c For $L e>1$ cases, Eq. 27) well reproduces the behavior of $\left\langle S_{r}^{*}+S_{n}^{*}\right\rangle_{\left(a_{T}^{*}, \kappa^{*}\right)}$ in Figs. 16d to 16f However, $\left\langle S_{r}^{*}+S_{n}^{*}\right\rangle_{\left(a_{T}^{*}, \kappa^{*}\right)}$ is overestimated (underestimated), when the tangential strain rate is strongly negative (positive). Fortunately, these over and under-estimations occur at extreme values of $a_{T}^{*}$ corresponding to very low probabilities according to the joint PDF.

Figure 17 shows the evolution of $\left\langle S_{r}^{*}+S_{n}^{*}\right\rangle_{\left(a_{T}^{*}, \kappa^{*}\right)}$ with $\kappa^{*}$ for the DNS and Eq. 27), considering a fixed $a_{T}^{*}$ corresponding to the maximum of joint PDF of $a_{T}^{*}$ and $\kappa^{*}$. In the same figure is plotted the PDF of $\kappa^{*}$ when $a_{T}^{*}$ is fixed. For $L e>1$ cases in Figs. 17d to 17f. Eq. 27] is in
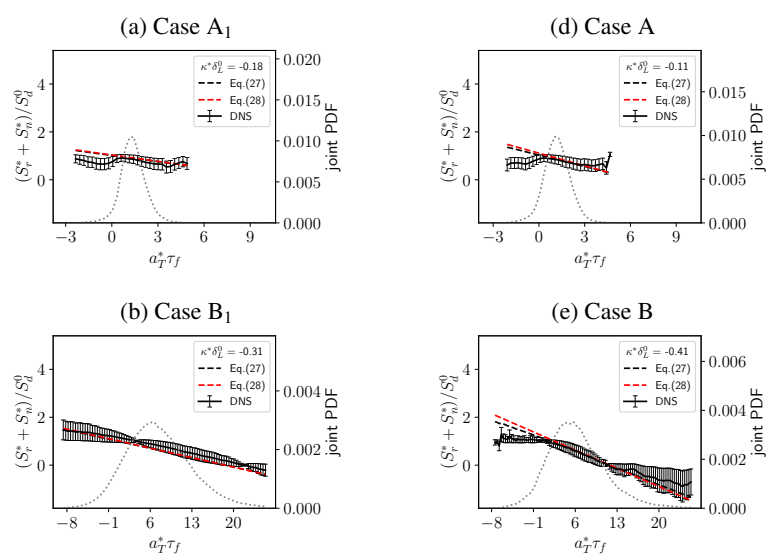

(c) Case $\mathrm{E}_{1}$
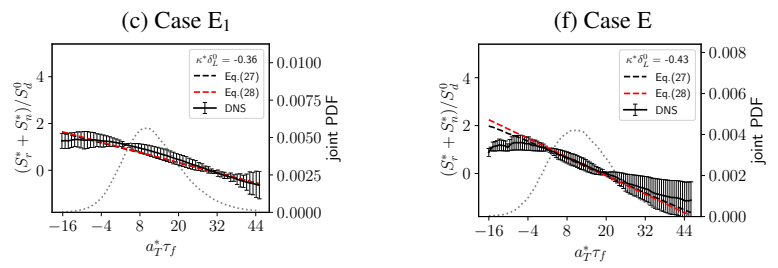

Figure 16: Evolution of $\left\langle S_{r}^{*}+S_{n}^{*}\right\rangle_{\left(a_{T}^{*}, \kappa^{*}\right)}$ from DNS and from Eqs. 27 and 28) with $a_{T}^{*}$ at a fixed $\kappa^{*}$ for: a to (c) the cases with $L e=1$ and (d) to (f) the cases with $L e>1$. The values of $\kappa^{*}$ chosen correspond to the maxima of joint PDF. The PDF of $a_{T}^{*}$ is shown in grey dot lines.
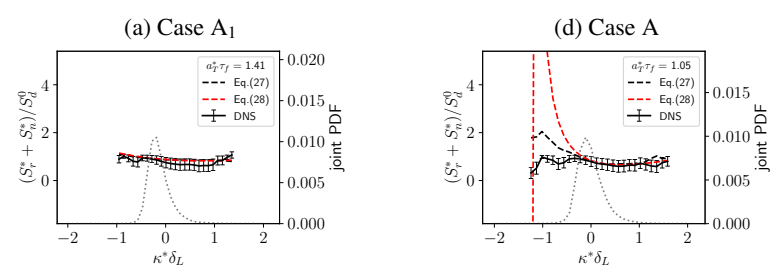

(b) Case $\mathrm{B}_{1}$

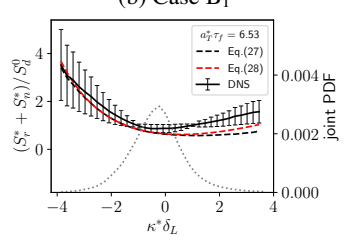

(e) Case B
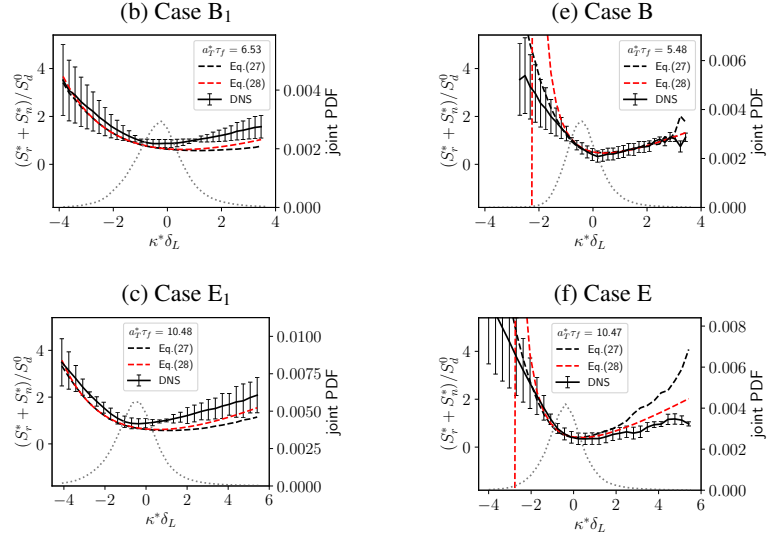

Figure 17: Evolution of $\left\langle S_{r}^{*}+S_{n}^{*}\right\rangle_{\left(a_{T}^{*}, \kappa^{*}\right)}$ from DNS and from Eqs. 27 and 28 with $\kappa^{*}$ at a fixed $a_{T}^{*}$ for: (a) to (c) the cases with $L e=1$ and (d) to ff the cases with $L e>1$. The values of $a_{T}^{*}$ chosen correspond to the maxima of joint PDF. The PDF of $\kappa^{*}$ is shown in grey dot lines. 
good agreement with DNS except for strongly negative and positive curvatures. For these extreme curvatures, Eq. 27) overestimates the velocity $\left\langle S_{r}^{*}+S_{n}^{*}\right\rangle_{\left(a_{T}^{*}, \kappa^{*}\right)}$. However, these over-estimations occur at extreme values of $\kappa^{*}$, corresponding to very low probabilities. Thus, these marginal values are rare and do not impact significantly the statistics of the displacement speed. For $L e=1$ cases in Figs. 17a and 17c the predictions of Eq. 27 are in good agreement with the DNS for negative curvatures. However, when the curvature becomes positive, Eq. (27) underpredicts the increase observed in the DNS. This leads to an underprediction of $\left\langle S_{r}^{*}+S_{n}^{*}\right\rangle_{\left(a_{T}^{*}, \kappa^{*}\right)}$ by a factor close to 2 for $\kappa^{*} \delta_{L} \geq 2$. Even if this underprediction is observed for curvature values corresponding to relatively low probabilities of $p\left(a_{T}, \kappa\right)$, it is expected that its impact on the mean prediction of $S_{d}^{*}$ by Eq. 27 will not be negligible.

In Figs. 16 and 17 the error bars correspond to the RMS of $\left\langle S_{r}^{*}+S_{n}^{*}\right\rangle$ in the DNS, at constant strain and curvature respectively. Unlike for the single conditioning on curvature shown in Fig. 12, these fluctuations remain very moderate, excepted at extreme strain and curvature values. This result justifies that $\left\langle S_{r}^{*}+S_{n}^{*}\right\rangle$ can be accurately modeled thanks to a function depending on curvature and strain, as proposed in Eq. 27).

\section{A priori modeling}

\subsection{Presentation of the model}

Section 4.4 suggests to model the progress variable and FSD source terms on a specific $c$ iso-surface. For this purpose we first define an adequate progress variable linked to the sole iso-surface $c=c^{*}$ as proposed by Knudsen and Pitsch [80]:

$$
C=\mathcal{H}\left(c-c^{*}\right),
$$

where $\mathcal{H}(c)$ is the Heaviside function giving $C=$ 0 when $c<c^{*}$ and $C=1$ otherwise. Knudsen and Pitsch [80] derived the transport equation for this progress variable:

$$
\frac{\partial \bar{\rho} \widetilde{C}}{\partial t}+\nabla \cdot(\overline{\rho \mathbf{u} C})=\left\langle\rho S_{d}\right\rangle_{s, c^{*}} \bar{\Sigma}^{*}
$$

where the flame surface density is defined as $\bar{\Sigma}^{*}=$ $\overline{|\nabla C|}=\overline{|\nabla c| \delta\left(c-c^{*}\right)}$ corresponding to the fine-grained FSD in [81]. The transport equation of the FSD then becomes:

$$
\begin{aligned}
\frac{\partial \bar{\Sigma}^{*}}{\partial t}+\nabla \cdot\left(\langle\mathbf{u}\rangle_{s, c^{*}} \bar{\Sigma}^{*}\right)=\left\langle a_{T}\right\rangle_{s, c^{*}} \bar{\Sigma}^{*} & +\left\langle S_{d} \nabla \cdot \mathbf{n}\right\rangle_{s, c^{*}} \bar{\Sigma}^{*} \\
& -\nabla \cdot\left(\left\langle S_{d} \mathbf{n}\right\rangle_{s, c^{*}} \bar{\Sigma}^{*}\right),
\end{aligned}
$$

where $\langle Q\rangle_{s, c^{*}}=\overline{Q|\nabla C|} / \bar{\Sigma}^{*}$ indicates surface averaging of any quantity $Q$ on the iso-surface $c=c^{*}$, and $\left\langle a_{T}\right\rangle_{s, c^{*}}=\langle\nabla \cdot \mathbf{u}-\mathbf{n n}: \nabla \mathbf{u}\rangle_{s, c^{*}}$ is the tangential strain rate.

The terms $\langle\mathbf{u}\rangle_{s, c^{*}} \bar{\Sigma}^{*}$ and $\overline{\rho \mathbf{u} C}$ in Eqs. 33 and 34 are not investigated here as this paper focuses on the source terms of $\bar{\Sigma}^{*}$. In addition, $\nabla \cdot\left(\left\langle S_{d} \mathbf{n}\right\rangle_{s, c^{*}} \bar{\Sigma}^{*}\right)$ denoting a laminar propagation term is expected to play a smaller role compared to turbulent strain rate, curvature and displacement speed in highly turbulent flows. This term is therefore not considered here either.

Starting from Eq. (28) we propose to express the mean displacement speed as the average over all possible curvature values:

$$
\left\langle\rho S_{d}\right\rangle_{s, c^{*}}=\rho^{*} \int_{\kappa_{\min }}^{+\infty} \frac{S_{d}^{0}-\mathcal{L}_{a_{T}}^{t}\left\langle a_{T}\right\rangle_{s, c^{*}}-D^{*} \kappa}{1+\mathcal{L}_{\kappa}^{t} \kappa} p(\kappa) d \kappa,
$$

where $p(\kappa)$ is the PDF of $\kappa$ defined later. As the integral in Eq. (35) is divergent at $\kappa=-1 / \mathcal{L}_{\kappa}^{t}$, the lower bound $\kappa_{\min }$ needs to be properly defined. The following expression is proposed:

$$
\kappa_{\min }=\max \left(\frac{\varepsilon-1}{\mathcal{L}_{\kappa}^{t}},-\frac{2}{\delta_{L}}\right)
$$

where $0<\varepsilon \ll 1$. This limitation of the integral means that the effect of curvatures $\kappa<\kappa_{\text {min }}$ is not accounted for. Figure 18 presents the integral of the pdf $p(\kappa)$ from $\kappa=\kappa_{\min }$ to $+\infty$ for $\varepsilon=0.1$ and 0.01 . As can be seen this integral is always very close to unity on the unburned side, and never goes below 0.98 on the burned gases side, which confirms that curvatures smaller than $\kappa_{\text {min }}$ are rare.

The first limit in Eq. (36) is numerical: at $\kappa=(\varepsilon-$ 1)/ $\mathcal{L}_{\kappa}^{t}$, the denominator in the integral is $\varepsilon$, chosen equal to 0.01 in the present study. This leads to very large values of $S_{d} / S_{d}^{0}$ which are far from the domain of validity of Eq. 28, , but which reflect the order of magnitude of $S_{d}$ that can be found on the burnt gases side of the flame when flame annihilation occurs as shown in [27]. The second limit is physical. It simply states that below a critical vortex size close to the inverse of the flame thickness, the flame cannot be curved anymore. Such a limit was proposed among others in the studies by 


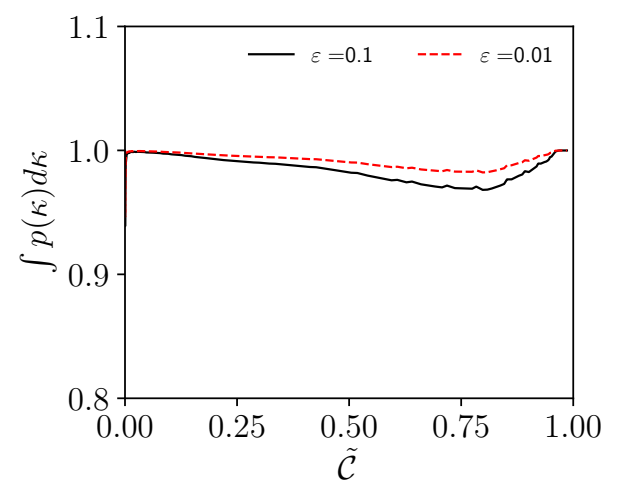

(a) Case $\mathrm{A}$

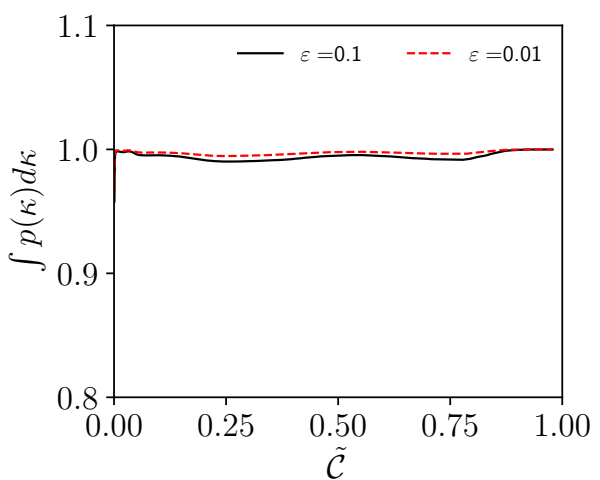

(b) Case B

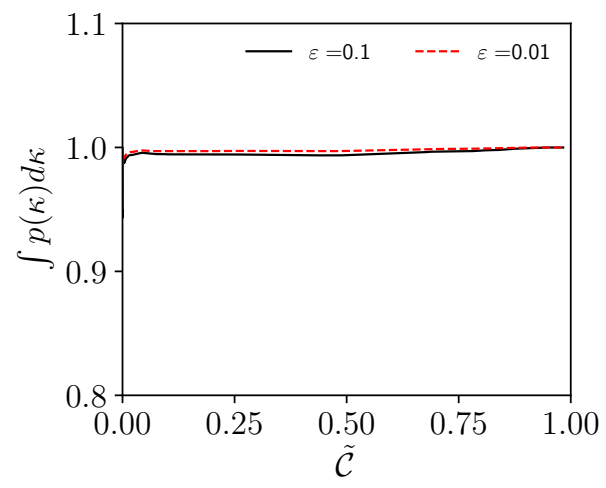

(c) Case $\mathrm{E}$

Figure 18: Evolution of the integral $\int_{\kappa_{\min }}^{+\infty} p(\kappa) d \kappa$ with $\widetilde{C}:$ a for case A, b for case B, and $(\mathrm{c}$ for case $\mathrm{E}$.

Poludnenko et al. [17] and Dave and Chaudhuri [27]. The former suggested that for local flame collisions the curvature is approximately $1 / \delta_{L}$. For the present turbulent flames, we observed that $-2 / \delta_{L}=-5194 \mathrm{~m}^{-1}$, corresponds roughly to the minimum curvature observed on the DNS in Fig. 20, which justifies the choice of this limit. Dave and Chaudhuri [27] proposed to model the effect of flame collisions on the displacement speed by analyzing the theoretical case of an inwardly propagating cylindrical flame. A new expression of $S_{d}$ was derived from this analysis. This expression could be used in future studies to model this quantity when large negative curvatures are found, replacing Eq. 28.

In order to evaluate the influence of the numerical bound, Fig. 19 presents $\left\langle S_{d}\right\rangle_{s, c^{*}}$ for all cases, for $\varepsilon=0.01$ and for 0.1 , that is, when a much larger fraction of negative curvatures are discarded from the integral. As seen in this figure, $\left\langle S_{d}\right\rangle_{s, c^{*}}$ remains very close for both values for cases $\mathrm{B}$ and $\mathrm{E}$ because we have $(\varepsilon-1) / \mathcal{L}_{\kappa}^{t}<-2 / \delta_{L}=-5194 \mathrm{~m}^{-1}$ leading to $\kappa_{\min }=-2 / \delta_{L}$, while for case $\mathrm{A}$, an acceptable variation is observed on the burned gases side. This result shows that the integration over curvatures larger than $\kappa_{\min }$ is quite robust from a modelling perspective.

At high Karlovitz numbers, strain rate and curvature become uncorrelated as shown in section 4.5. We then assume that in Eq. (35) $\left\langle a_{T}\right\rangle_{s, c^{*}}$ does not depend on curvature. At low Karlovitz numbers on the other hand, the impact of strain on the displacement speed becomes small as it becomes nearly uniform and close to $S_{d}^{0}$. Therefore, although less justified, assuming a constant turbulent strain rate in Eq. 35 should not significantly alter the prediction of the model in this regime.

The PDF $p(\kappa)$ is now presumed to follow a Gaussian distribution:

$$
p(\kappa)=\frac{1}{\sqrt{2 \sigma^{2} \pi}} \exp \left(-\frac{(\kappa-\mu)^{2}}{2 \sigma^{2}}\right),
$$

where $\mu=\langle\kappa\rangle_{s, c^{*}}$ is the surface average of $\kappa$ and $\sigma^{2}=\left(\left\langle\kappa^{2}\right\rangle_{s, c^{*}}-\langle\kappa\rangle_{s, c^{*}}^{2}\right)$ the corresponding variance.

Figure 20 compares the PDF extracted from the DNS and the modeled one, using $\langle\kappa\rangle_{s, c^{*}}$ and $\left\langle\kappa^{2}\right\rangle_{s, c^{*}}$ extracted from the DNS to compute $\mu$ and $\sigma^{2}$, for cases B and 


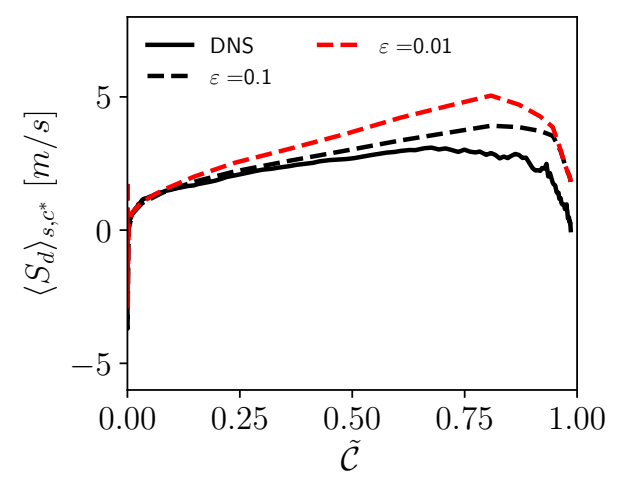

(a) Case A

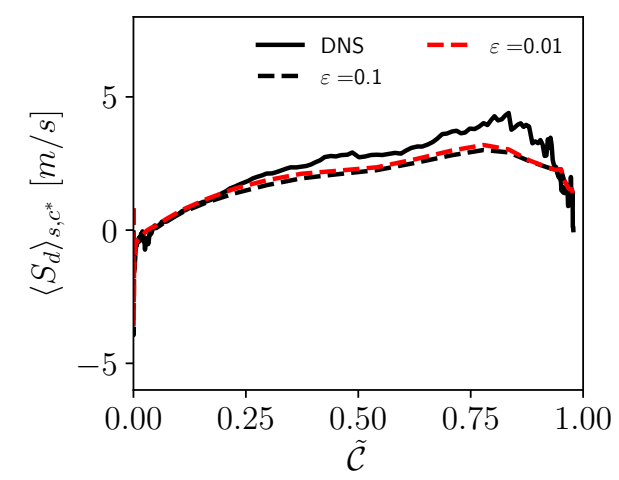

(b) Case B

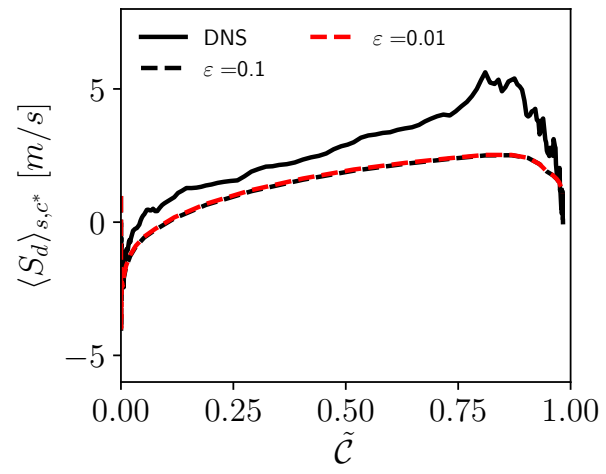

(c) Case $\mathrm{E}$

Figure 19: Evolution of the displacement speed $\left\langle S_{d}\right\rangle_{s, c^{*}}$ with $\widetilde{C}:$ a for case A, b for case B, and $[\mathrm{c}$ for case E.

$\mathrm{B}_{1}$ at three positions in the flame front corresponding to $\widetilde{C}=0.2,0.5$ and 0.8 . The agreement is good although the model tends to over-predict the PDF width.

A model for $\left\langle S_{d} K\right\rangle_{s, c^{*}}$ is simply deduced from that of $\left\langle S_{d}\right\rangle_{s, c^{*}}$ by multiplying the integral of Eq. 35 by $\kappa$ :

$$
\left\langle S_{d} \kappa\right\rangle_{s, c^{*}}=\int_{-\frac{1}{\mathcal{L}_{\kappa}^{t}}+\varepsilon}^{+\infty} \frac{S_{d}^{0} \kappa-\mathcal{L}_{a_{T}}^{t}\left\langle a_{T}\right\rangle_{s, c^{*}} \kappa-D^{*} \kappa^{2}}{1+\mathcal{L}_{\kappa}^{t} \kappa} p(\kappa) d \kappa
$$

To close Eqs. 35, 37) and 38), models are needed for $\langle\kappa\rangle_{s, c^{*}}$ and $\left\langle\kappa^{2}\right\rangle_{s, c^{*}}$ :

- The curvature is modeled using the model proposed by Rymer [48] (see also [22]) adapted to Eqs. 33. and (34).

$$
\langle\kappa\rangle_{s, c^{*}}=\beta_{1} \frac{c_{1}-\bar{C}}{\bar{C}(1-\bar{C})}\left(\bar{\Sigma}^{*}-|\nabla \bar{C}|\right),
$$

where $\beta_{1}=4 / 3$ and $c_{1}=0.5$ are the model parameters used in [22].

- For $\left\langle\kappa^{2}\right\rangle_{s, c^{*}}$ an expression similar to Eq. 39] is pro- posed:

$$
\left\langle\kappa^{2}\right\rangle_{s, c^{*}}=\beta_{2} \frac{1}{\bar{C}^{2}(1-\bar{C})^{2}}\left(\bar{\Sigma}^{*}-|\nabla \bar{C}|\right)^{2},
$$

where $\beta_{2}$ is a model parameter set to 1 .

\subsection{A priori tests of models for tangential strain rate}

In this section, existing models for the tangential strain rate in the flamelet regime are evaluated against the present DNS. Cant and Pope [82], suggest that strain rate is inversely proportional to the Kolmogorov time scale $\tau_{\eta}$. Considering the definition of the Karlovitz number as the ratio of chemical time scale to Kolmogorov time scale, the strain rate can be expressed as a function of $K a$ :

$$
\left\langle a_{T}\right\rangle_{s, c^{*}}=\alpha_{C a n t} K a \frac{S_{L}^{0}}{\delta_{L}},
$$

where $\alpha_{\text {Cant }}$ is a model parameter.

Another approach consists in modeling the turbulent strain rate thanks to $2 \mathrm{D}$ DNS of flame/vortex interactions, following Meneveau and Poinsot [83]. Using this 


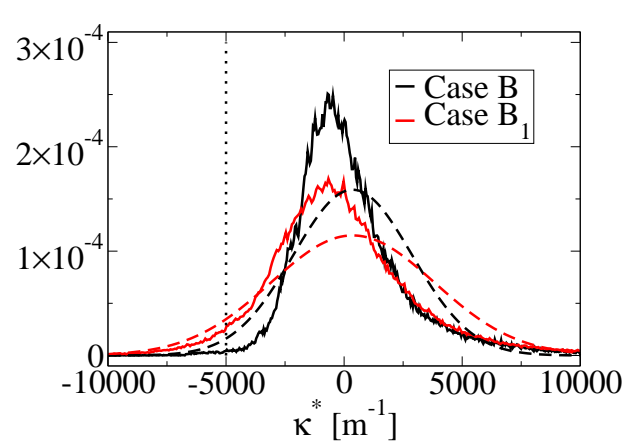

(a) $\widetilde{C}=0.2$

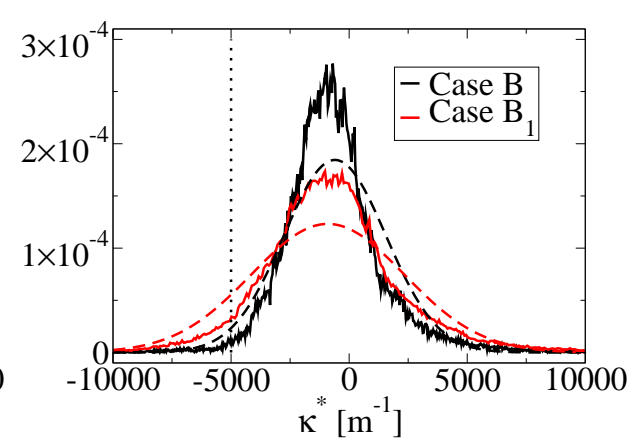

(b) $\widetilde{C}=0.5$

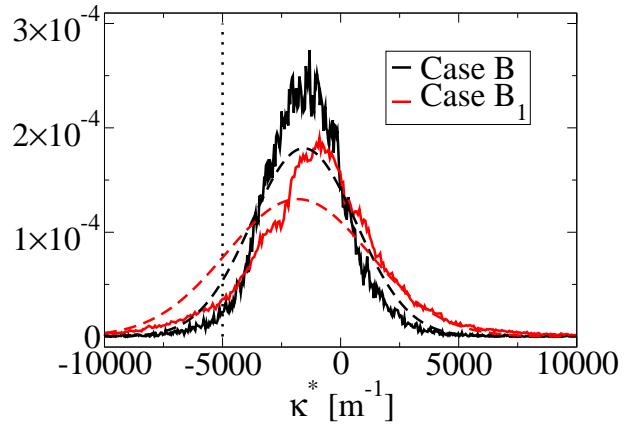

(c) $\widetilde{C}=0.8$

Figure 20: Probability density function of $\kappa$ in solid line compared to a presumed Gaussian PDF, defined in Eq. 37 in dashed line: a) for $\widetilde{C}=0.2$, b) for $\widetilde{C}=0.5$ and $\widetilde{c}$ for $\widetilde{C}=0.8$. The vertical dotted line represents the value of $k$ where $1+\mathcal{L}_{k} k$ is zero for the case $\mathrm{B}$ (for the case $\mathrm{B}_{1}$ this value is approximately $-20000 \mathrm{~m}^{-1}$ ).

approach, Charlette et al. [84] expressed the turbulent strain rate as the product of the strain at the integral scale $u^{\prime} / l_{t}$ by a factor $\Gamma$ accounting for the efficiency of all turbulent scales:

$$
\left\langle a_{T}\right\rangle_{s, c^{*}}=\alpha_{a_{T}} \Gamma \frac{u^{\prime}}{l_{t}},
$$

where $\alpha_{a_{T}}$ is a modeling constant. Function $\Gamma$ is defined from the relationship existing between strain rate and energy spectrum in homogeneous turbulence:

$$
\left(\Gamma \frac{u^{\prime}}{l_{t}}\right)^{2}=\left(\frac{\pi}{l_{t}}\right)^{3} \int_{1}^{\infty}\left[C\left(k_{+}\right)\right]^{2} k_{+}^{2} E_{11}\left(k_{+}\right) d k_{+},
$$

where $k_{+}=k l_{t} / \pi$ is the dimensionless wavenumber and $C\left(k_{+}\right)$is an efficiency function, which takes into account the ability of the turbulent eddies at scale $k$ to stretch the flame. $E_{11}\left(k_{+}\right)$is the one-dimensional (longitudinal) energy spectrum in the direction of the wavenumber $k$, defined using the standard longitudinal Kolmogorov spectrum with the Pao correction to account for the viscous cut-off:

$$
E_{11}\left(k_{+}\right)=\frac{18}{55}\left(\pi \frac{k_{+}}{l_{t}}\right)^{-5 / 3} \varepsilon^{2 / 3} \exp \left(-\frac{3}{2} C_{k}\left(\pi k_{+} \frac{\eta}{l_{t}}\right)^{4 / 3}\right)
$$

where $C_{k} \approx 1.5$ is the universal Kolmogorov constant, $\eta$ is the Kolmogorov scale and $\varepsilon$ is the rate of dissipation of TKE.

Thus, in Charlette's approach, the key parameter to model is the efficiency $C\left(k_{+}\right)$. A first assumption is to consider that all scales stretch the flame front with the same efficiency, which reads $C\left(k_{+}\right)$constant chosen here as $C\left(k_{+}\right)=1$. Equation (42) then becomes, by combining Eqs. (43) and 447:

$$
\left\langle a_{T}\right\rangle_{s, c^{*}}=\alpha_{a_{T}}\left(\frac{3}{\sqrt{55}}\right) \sqrt{\frac{R e_{t}}{C_{k}}} \sqrt{\exp \left(-\frac{3}{2} C_{k} \frac{\pi^{4 / 3}}{R e_{t}}\right)}\left(\frac{u^{\prime}}{l_{t}}\right) .
$$

Yet, $\left(u^{\prime} / l_{t}\right) \sqrt{R e_{t}}=\left(u^{\prime} / l_{t}\right) D a K a=\left(S_{L}^{0} / \delta_{L}\right) K a$. In addition, by assuming that $R e_{t}$ is sufficiently large, $\exp \left(-(3 / 2) C_{k} \pi^{4 / 3} / R e_{t}\right)$ is close to 1 . Thus, by combining $\alpha_{a_{T}}$ and $1 / \sqrt{C_{k}}$, the model for the tangential strain 
rate is:

$$
\left\langle a_{T}\right\rangle_{s, c^{*}}=\alpha_{a_{T}} \frac{3}{\sqrt{55}} K a \frac{S_{L}^{0}}{\delta_{L}} .
$$

Equation (46) shows that Charlette's model becomes equivalent to Cant's model when all scales are considered to have the same efficiency. However, as already demonstrated by several authors [20, 83, 85], assuming a constant efficiency is not realistic because turbulent scales smaller than the flame thickness $\delta_{L}$ might be too small to strain the flame significantly.

This is why Charlette et al. [84] and Bougrine et al. [85] proposed algebraic expressions for $C\left(k_{+}\right)$(not shown here) based on a best-fit of their flame/vortex DNS. Their models for strain are commonly used in engine applications using CFM as a combustion model.

Here, an alternative solution is proposed: $C\left(k_{+}\right)$is modeled as an Heaviside function with a cut-off scale $\delta_{c}$, so that $C\left(k_{+}\right)=1$ when $k_{+}<l t / \delta_{c}$ and $C\left(k_{+}\right)=0$ otherwise. Similarly to $C\left(k_{+}\right)=1$, this function allows to simplify Eq. (43) leading to the following, when combined with Eq. (42):

$\left\langle a_{T}\right\rangle_{s, c^{*}}=\alpha_{a_{T}} \frac{3}{\sqrt{55}} K a \frac{S_{L}^{0}}{\delta_{L}}\left[1-\exp \left(-\frac{3}{2} C_{k} \frac{1}{R e_{t}}\left(\frac{\pi l_{t}}{\delta_{c}}\right)^{4 / 3}\right)\right]^{1 / 2}$.

This approach considers the flame as a low-pass filter (LPF) in term of turbulence wavenumber. The cut-off scale $\delta_{c}$ is identified to the laminar flame thickness in the following. This value has been chosen to best reproduce the tangential strain rate of the DNS. This model is designated in the rest of this study as the LPF model.

A comparison of $\left\langle a_{T}\right\rangle_{s, c^{*}}$ extracted from the DNS with Cant's (Eq. (41)), Charlette's [84] and Bougrine's models [85] and with the LPF model (Eq. 477) is shown in Fig. 21 for all DNS cases. The model parameter $\alpha_{a_{T}}$ appearing in each model is fixed using Charlette's model [84]. This parameter is adjusted to fit the strain rate obtained at the lowest Karlovitz numbers (i.e., cases $\mathrm{A}$ and $\mathrm{A}_{1}$ ). The values of $\alpha_{a_{T}}$ used are 1.65 and 2.81 for $L e>1$ and $L e=1$, respectively. The same factors are applied to the other models for consistency, except for Bougrine's model. Indeed Bougrine's function is the only one to include Lewis number effect through a corrective function. Therefore a unique parameter is used for all cases which is the one set for Charlette on $L e=1$. In addition, the values predicted by Bougrine's function being much smaller than the other models, they are multiplied by a factor 2.5 in Fig. 21 for better visibility.

First looking at $L e>1$ cases in Fig. 21b. Cant's model presents a linear increase of $\left\langle a_{T}\right\rangle_{s, c^{*}}$ with $K a$, as

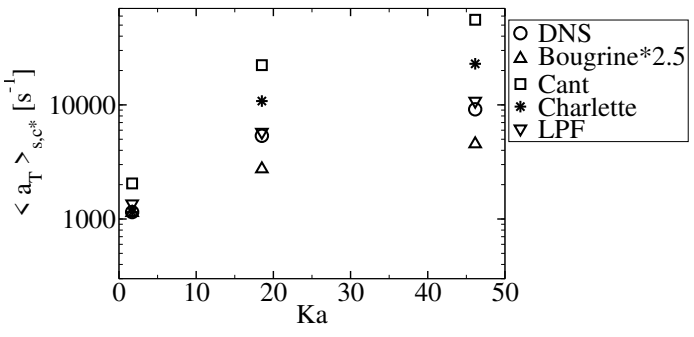

(a) $L e=1$

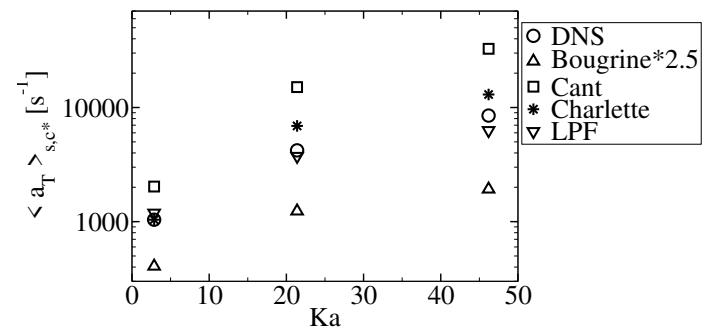

(b) $L e>1$

Figure 21: Tangential strain rate $\left\langle a_{T}\right\rangle_{s, c^{*}}$ from DNS compared to Bougrine's model [85], Cant's model [82], Charlette's model [84] and LPF model: a for cases with $L e=1$ and (b) for cases with $L e>1$.

expected from Eq. 46, in agreement with the DNS. The turbulent strain rate predicted in case A is twice as large as that of the DNS. In addition, the slope of this line is too steep compared to the DNS. As a consequence, very large discrepancies are observed for high Karlovitz numbers. For instance the predicted strain rate in case $\mathrm{E}$ is almost four times larger than that of the DNS. Charlette's model over-predicts the turbulent strain rate for high Karlovitz numbers, with up to $64 \%$ relative error in case B. Nevertheless, the general trend with $K a$ seems to be well reproduced, contrary to Cant's model. Surprisingly, Bougrine's model significantly under-predicts strain rates: for each case the predicted strain rate is about half the one of the DNS. This might be due to the strong decrease of the efficiency function for small turbulent structures, as seen in [85]. Nevertheless, the relative evolution of Bougrine's model is in good agreement with the DNS. Note that Bougrine's turbulent strain rate flattens with increasing $K a$, while the DNS strain remains quasi-linear with $K a$. This might be explained by a too strong decrease of Bougrine's efficiency function with the vortex velocity [85]. The Lewis number dependence of Bougrine's function is not sufficient to recover the Lewis number influence in DNS results. LPF model is the only one to accurately predicts the turbulent strain rate for each case, with a maximum relative error of $25 \%$ for case $\mathrm{E}$.

For $L e=1$ cases shown in Fig. 21a the predictions of each model are similar to non-unity Lewis number 
cases. As for $L e>1$ cases, Bougrine's model strongly underestimates $\left\langle a_{T}\right\rangle_{s, c^{*}}$ by a factor 2.5 and up to a factor 5 for cases $A_{1}$ and $E_{1}$, respectively. Note that predictions of Cant's and Charlette's models are deteriorated compared to $L e>1$ cases. The latter presents a relative error of $150 \%$ for the highest Karlovitz number. Nevertheless, the main conclusions here are the same as for the non-unity Lewis number simulations.

Present results obtained with Charlette's and Bougrine's models at high Karlovitz confirm that the inability of small eddies to stretch the flame needs to be included in turbulent strain models at all turbulence levels. It also shows that choosing $\delta_{L}$ as the cut-off scale seems appropriate for the LPF model. From these cases, the LPF model seems to give the better prediction for $\left\langle a_{T}\right\rangle_{s, c^{*}}$ with a maximum error always smaller than $25 \%$. Nevertheless, this cut-off length might also be dependent on $K a$, as already suggested for low $K a$ by Gülder and Smallwood [86].

\subsection{A priori tests of models for curvature and displace- ment speed}

As a first step, the a priori modeling of the mean of curvature and square of curvature are evaluated against the DNS, following the averaging procedure described in Appendix D.

\section{Models for curvature}

Rymer's closure, Eq. 39 , for $\langle\kappa\rangle_{s, c^{*}}$ is first examined. The values of $\bar{C}, \Sigma^{*}$ and $|\nabla \bar{C}|$ used to compute $\langle\kappa\rangle_{s, c^{*}}$ are the exact values from DNS. The comparison in Fig. 22 shows a good agreement between the model and the DNS, especially for $L e=1$ cases. In addition, although this closure was proposed for the flamelet regime, it becomes even more accurate as the Karlovitz number increases. The values of the model parameter $c_{1}$ provided by Rymer [48] is based on the assumption of symmetric profiles of $\bar{\Sigma}$ centered on 0.5 with regards to $\bar{c}$. The unity Lewis number cases present quite symmetric profiles of $\bar{\Sigma}$, which explains the very good agreement between the model and DNS for these cases. The profiles of $\bar{\Sigma}$ with $\bar{c}$ for $L e>1$ cases are less symmetric explaining the reduced agreement with DNS. However, when $K a$ increases the profiles of $\bar{\Sigma}$ tend to get closer to the profiles of $L e=1$ cases. This phenomenon explains the better prediction of Rymer's model for high Karlovitz number flames.

Then, the model for $\left\langle\kappa^{2}\right\rangle_{s, c^{*}}$, Eq. 40, is compared in Fig. 23 to the DNS and to the model proposed by
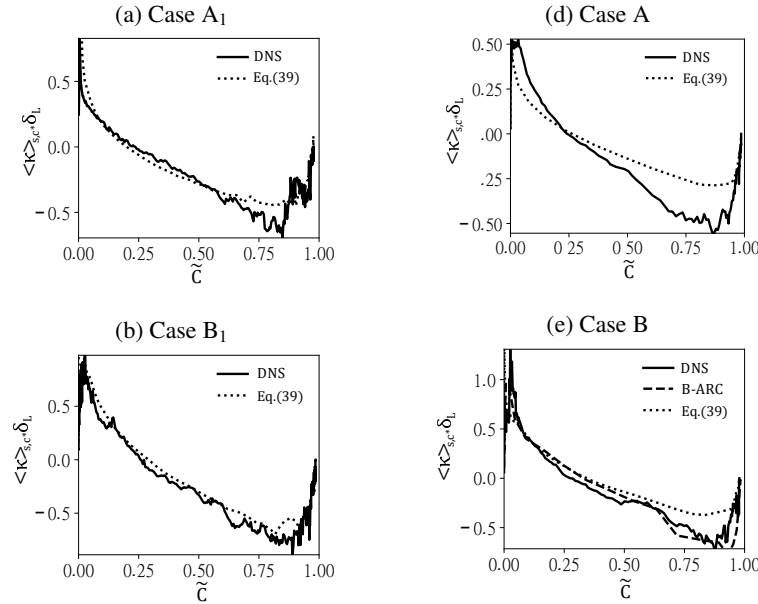

(c) Case $\mathrm{E}_{1}$
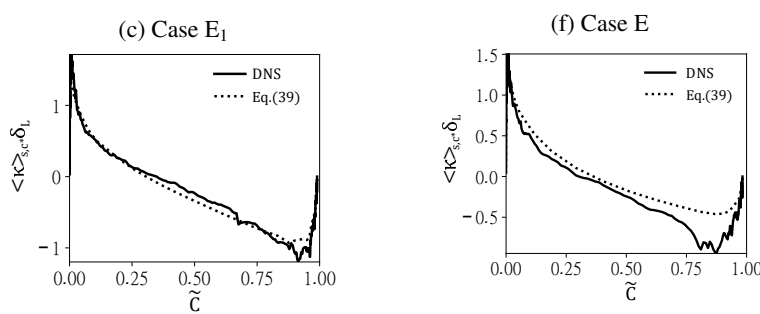

Figure 22: Comparison of the model for $\langle\kappa\rangle_{s, c^{*}}$ given by Eq. 39] with the DNS data versus $\widetilde{C}$ for: a to (c) cases with $L e=1$ and $(\mathrm{d})$ to $f$, cases with $L e>1$.

Hawkes et al. in [87]:

$$
\left\langle\kappa^{2}\right\rangle_{s, c^{*}}=\left(1-\left\langle n_{k}\right\rangle_{s, c^{*}}\left\langle n_{k}\right\rangle_{s, c^{*}}\right)^{2} \frac{\bar{\Sigma}^{* 2}}{\bar{C}^{2}(1-\bar{C})^{2}},
$$

where $\left\langle n_{k}\right\rangle_{s, c^{*}}=-\nabla \bar{C} / \bar{\Sigma}^{*}$ is the $k^{\text {th }}$ component of the surface averaged flame normal vector.

For non-unity Lewis number cases, the order of magnitude of $\left\langle\kappa^{2}\right\rangle_{s, c^{*}}$ is well reproduced by Eq. 40 . For case A, the model tends to under-predict the strong increase of $\left\langle\kappa^{2}\right\rangle_{s, c^{*}}$ towards $\widetilde{C}=0$ while it correctly recovers the decrease of $\left\langle\kappa^{2}\right\rangle_{s, c^{*}}$ when $\widetilde{C} \geq 0.8$. For cases B and E, the agreement with the DNS is much better. Note that the oscillations observed on the DNS results for $\widetilde{C}$ close to zero and one might be due to the small samples available for statistics in these regions and to the very large curvatures observed in these regions, leading to poor numerical resolution. For the unity Lewis number cases, the agreement with the DNS is very good at all Karlovitz numbers.

Hawkes's model presents similar results at large Karlovitz numbers, which is not surprising as both assume similar expressions. At the same time, Hawkes 

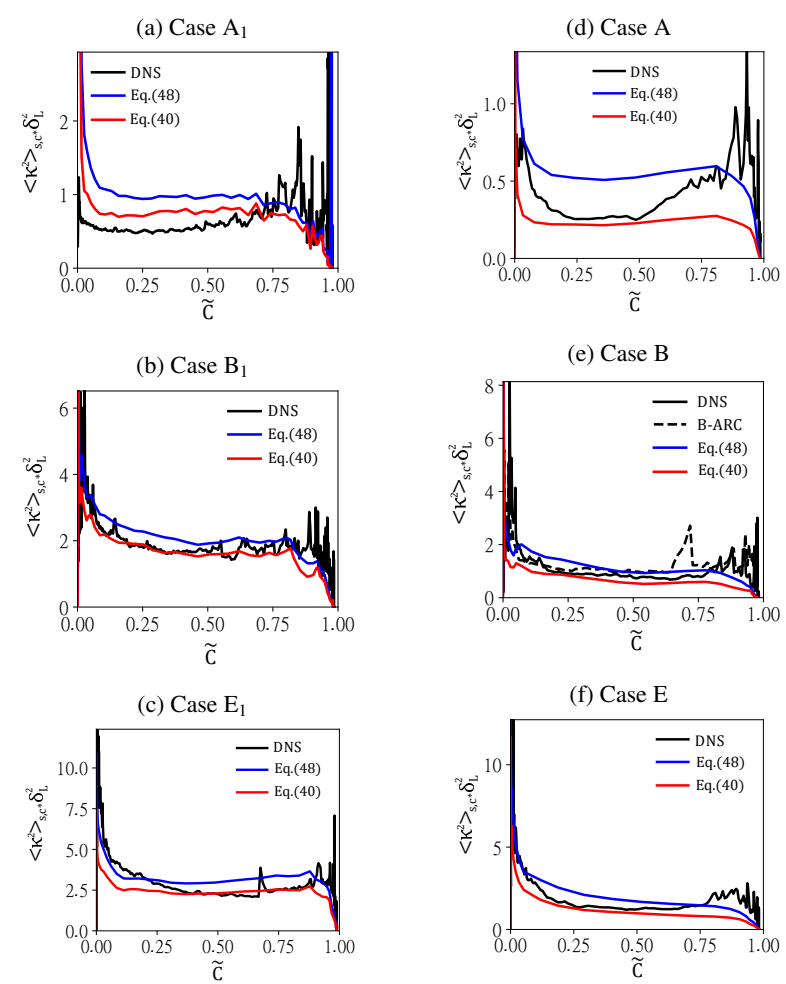

Figure 23: Comparison of the model for $\left\langle\kappa^{2}\right\rangle_{s, c^{*}}$ with the DNS data and the model proposed by Hawkes et al. [87] versus $\widetilde{C}$ for: a to [c] cases with $L e=1$ and (d) to ff cases with $L e>1$.

model tends to over-predict $\left\langle\kappa^{2}\right\rangle_{s, c^{*}}$ by a factor of three for case A, unlike the proposed model.

Finally, in Fig. 22e and 23e $\langle\kappa\rangle_{s, c^{*}}$ and $\left\langle\kappa^{2}\right\rangle_{s, c^{*}}$ obtained for the DNS using the ARC mechanism are also presented. Qualitatively, very similar tendencies are obtained with case B and B-ARC. Discrepancies can be quantified between both results. At $\tilde{C}=0.5$ the relative error on $\left\langle\kappa^{2}\right\rangle_{s, c^{*}}$ obtained with the 2-step mechanism is $26 \%$ in Fig. $23 \mathrm{e}$ compared to the results obtained with the ARC mechanism (ISOOCT18). Moreover the mean relative error in the range $0.1<\tilde{C}<0.9$ reaches $21 \%$ which is still quite acceptable. Finally we can conclude that flame curvature statistics are weakly affected by the chemistry description and that the 2-step description remains an acceptable approximation of chemistry on this aspect.

\section{Models for the stretch due to curvature}

The proposed model for the stretch due to curvature, defined by Eq. (38), is compared to the DNS in Fig. 24 The Markstein lengths correlations for $\mathcal{L}_{a_{T}}^{t}$ and $\mathcal{L}_{\kappa}^{t}$ given by Eq. (30) and Eq. (31), respectively, are used. In this figure is also presented a model proposed by Katragadda
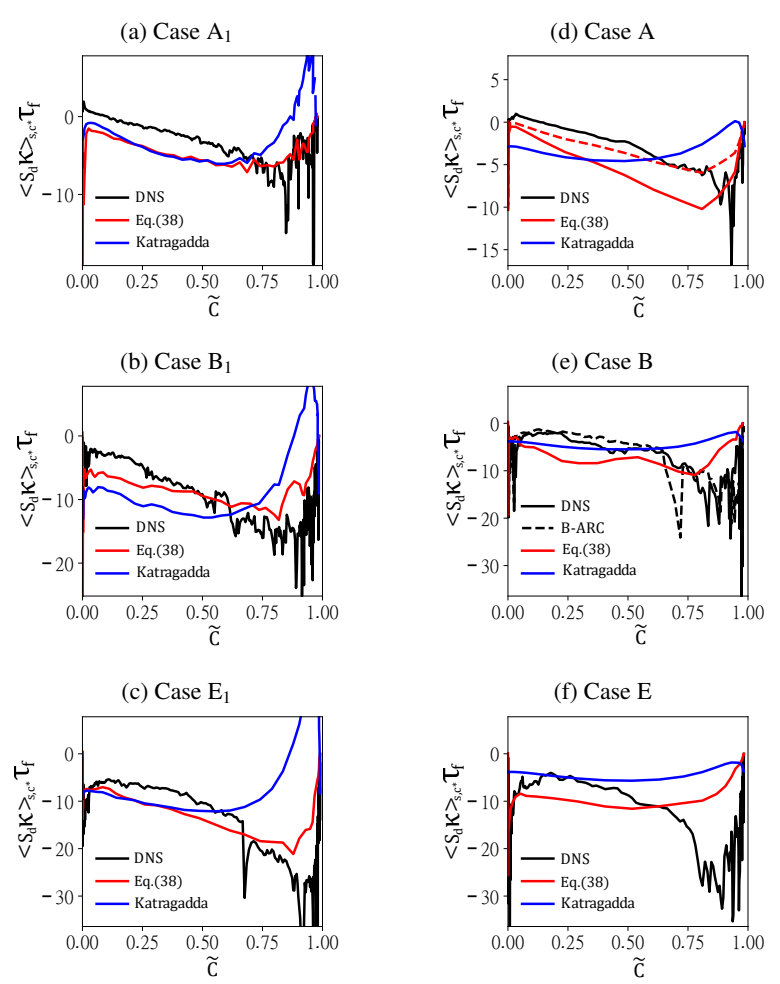

Figure 24: Comparison of the stretch due to curvature $\left\langle S_{d} \kappa\right\rangle_{s, c^{*}}$ for Katragadda's model and the proposed model with the DNS versus $\widetilde{C}$ for: (a) to (c) cases with $L e=1$ and (d) to (f) cases with $L e>1$. In (d) the red dashed line correspond to the model using the Markstein lengths directly fitted on the DNS.

[38] (given in Appendix E), using their suggested values for the model parameters. Although developed for the generalized approach, Katragadda's model is here computed using quantities $\bar{\Sigma}^{*}$ and $\bar{C}$ on the iso-surface $c^{*}$.

For $L e=1$ cases, Fig. 24a to 24c, the proposed model presents a good agreement with the DNS by reproducing the decrease of $\left\langle S_{d} \kappa\right\rangle_{s, c^{*}}$ with $\widetilde{C}$. For these cases, Katragadda's model is close to the proposed model excepted on the burned gases side where it presents large positive values while the DNS remains negative. For $L e>1$ cases, the proposed model reproduces qualitatively the evolution of $\left\langle S_{d} \kappa\right\rangle_{s, c^{*}}$ as seen in Fig. 24d to 24f. In particular, the model is in good agreement with the DNS towards $\widetilde{C}=0$ and the decrease of $\left\langle S_{d} \kappa\right\rangle_{s, c^{*}}$ with $\widetilde{C}$ up to 0.8 approximately is correctly reproduced. On the contrary, above this value, the model goes back to zero too quickly compared to the DNS. This discrepancy can partly be explained by the strong numerical noise observed in the DNS on the burned gases side as mentioned previously. Katragadda's model also recov- 
ers the order of magnitude of $\left\langle S_{d} \kappa\right\rangle_{s, c^{*}}$ but it is less accurate than the proposed model and shows a too flat profile compared to the DNS. Although qualitative agreement between the DNS data and Eq. [38, as well as with Katraggada's model [38] is obtained, an accurate description of $\left\langle S_{d} K\right\rangle_{s, c^{*}}$ is still not reached.

In Fig. 24d, the proposed model is also presented in red dashed line using the Markstein lengths fitted from DNS case A in section 4.5. The model prediction improves remarkably on the burnt gases side with these Markstein lengths. For instance at $\widetilde{C}=0.8$ the model using the correlation over-estimates $\left\langle S_{d}\right\rangle_{s, c^{*}}$ by a factor two while when using the fitted values, the error goes to nearly zero. This result shows that the proposed model has the ability to correctly recover the DNS results but it also shows that unfortunately, the model prediction is highly sensitive to the turbulent Markstein lengths employed. Future work is certainly needed to better assess how these lengths could be defined in a more reliable and possibly universal manner.

DNS case B using the ARC mechanism is also presented in Fig. 24e. Unlike previous flame statistics, quantitatively, the difference for $\left\langle S_{d} \kappa\right\rangle_{s, c^{*}}$ versus $\tilde{C}$ is quite large between the two mechanisms: for $0.25<$ $\tilde{C}<0.6$, the mean and maximum relative difference on $\left\langle S_{d} \kappa\right\rangle_{s, c^{*}}$ between the two mechanisms is about $40 \%$ and $60 \%$ respectively. Nevertheless, the two curves are very similar in terms of shape, indicating that presumably the physical mechanisms at stake are identical and thus not modified by the choice of the chemistry.

\section{Models for displacement speed}

Predictions of $\left\langle S_{d}\right\rangle_{s, c^{*}}$ by the proposed model in Eq. (35), are now assessed against the DNS and the constant $\left\langle S_{d}\right\rangle_{s, c^{*}}$ model adopted in the standard flamelet models $\left(\left\langle\rho S_{d}\right\rangle_{s, c^{*}}=\rho_{u} S_{L}\right)$. Fig. 25d presents $\left\langle S_{d}\right\rangle_{s, c^{*}}$ given by the proposed model for case A, using both the Markstein lengths fitted from the DNS (in dotted line) and those given by Eqs. 30 and (31). It can be seen that $\left\langle S_{d}\right\rangle_{s, c^{*}}$ is over-predicted when correlations are used, while it closely follows the DNS for $\widetilde{C}>0.1$ with the fitted values. This result is in agreement with what was observed for $\left\langle S_{d} \kappa\right\rangle_{s, c^{*}}$ and confirms the strong impact of Markstein length evaluations on both quantities. Note that the error observed with the Markstein length correlation is maximum for case A, which is the case where the correlation shows the poorest agreement with the fitted values as seen in Fig. 15. On the contrary for cases B and E, a much smaller difference is observed between fitted and correlation values (not shown).

For all six DNS cases, the proposed model predicts globally the increase of $\left\langle S_{d}\right\rangle_{s, c^{*}}$ with $\widetilde{C}$ observed in the
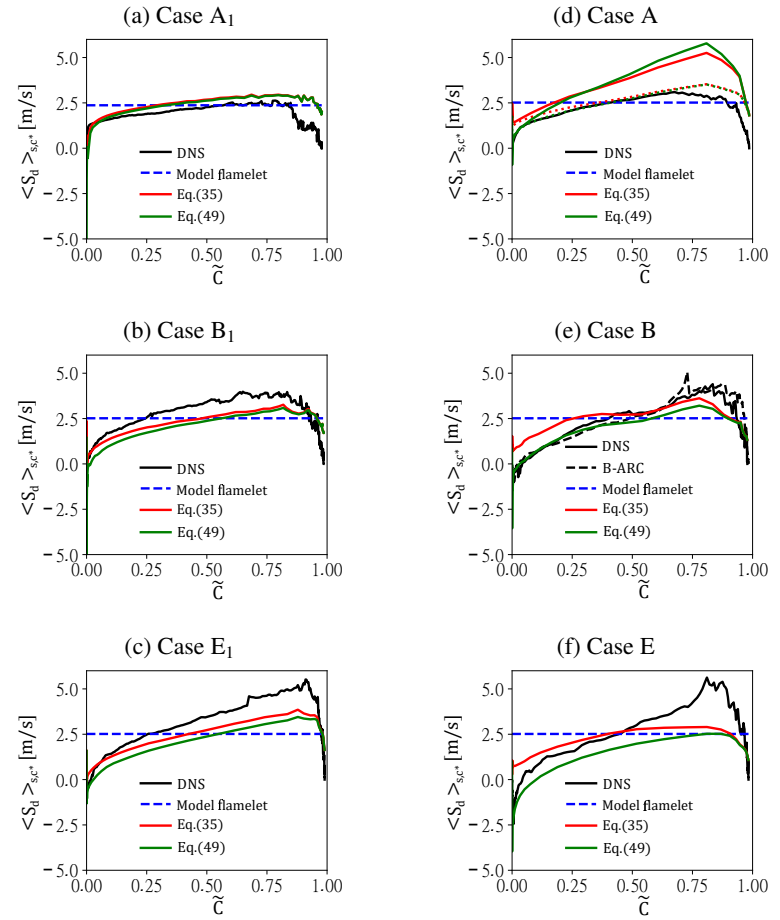

Figure 25: Comparison of the displacement speed $\left\langle S_{d}\right\rangle_{s, c^{*}}$ for the proposed models in Eqs. 35 and 49 with the DNS versus $\widetilde{C}$ for: a to (c) cases with $L e=1$ and (d) to (f) cases with $L e>1$. In (d) the dotted line correspond to the model using the Markstein lengths directly fitted on the DNS results.

DNS, unlike the flamelet model which remains constant, which makes a substantial improvement. At the same time this increase can be substantially over (case A) or under (cases B and E) predicted on the burned gases side. The model proposed with Eq. (35) is based on the presumed PDF of curvature (Eq. (37)), which is evaluated knowing $\langle\kappa\rangle_{s, c *}$ and $\left\langle\kappa^{2}\right\rangle_{s, c *}$. Yet, Figs. 22 and 23 show significant errors on the prediction of these two variables close to the burned gases side, like for instance at $\tilde{C}=0.8$. In addition, the Gaussian PDF is shown to be slightly larger than the PDF of the DNS (Fig. 20c), and symmetric, unlike the DNS. The combination of these approximations is certainly responsible for the largest part of the under and over predictions observed in Fig. 25.

More importantly from a modeling perspective, the proposed model under-predicts the strong decrease of $\left\langle S_{d}\right\rangle_{s, c^{*}}$ when $\widetilde{C}$ tends toward zero for non-unity Lewis number cases: for case $\mathrm{A},\left\langle S_{d}\right\rangle_{s, c^{*}}$ form the DNS approaches zero in this zone and becomes even negative for cases B and E. The main explanation for this error is that the proposed model relies on Eq. 28) for the dis- 
placement speed, which is directly taken from laminar flame analysis [27, 88]. This expression assumes that the perturbation wavelength is much larger than the laminar flame thickness and that the displacement speed is locally in equilibrium with the local stretch. However, all the cases considered here present a Karlovitz number larger than unity, therefore a laminar flame thickness greater than the Kolmogorov length scale ( $K a=$ $\left.\left(\delta_{L} / \eta\right)^{2}\right)$. Consequently, the assumption on the wavelength is not satisfied and the equilibrium assumption might not be valid any more and the flame responds to stretch in a delayed and attenuated way [79].

The discussion above suggests that the instantaneous displacement speed $S_{d}$ of the flame is an attenuated response to the stretch history seen by the flame along its movements across the flame brush. Taking into account this attenuation and history is a real modelling challenge. As a first step towards such a model, we found that considering in the linear model Eq. 27) an effective stretch $S_{d}^{0} \kappa$ instead of the instantaneous one, $S_{d} \kappa$, allowed to substantially improve the prediction of $S_{d}$ for $\kappa>0$. This modification does not constitute a reliable and well justified model, it only illustrates the limits of Eq. 27) on highly turbulent flows and suggests theoretical research work is necessary to account for the flame response time to turbulence. Interestingly enough, very recently, Dave and Chaudhuri [27] proposed a very similar hypothesis for the modelling of $S_{d}$ on the unburned gas side (Eq. (3.6) in their paper, equivalent to the expression of $S_{d}$ in Eq. (49) below for the case $\kappa>0$ ), to replace the reference expression involving a denominator (Eq. (3.2) in their paper, equivalent to Eq. (28) in this study). Mathematically, this leads to the integration of two different expressions of $S_{d}$ for $\kappa>0$ and $\kappa<0$ :

$$
\begin{aligned}
\left\langle\rho S_{d}\right\rangle_{s, c^{*}}= & \rho^{*} \int_{\kappa_{\min }}^{0}\left[\frac{S_{d}^{0}-\mathcal{L}_{a_{T}}^{t}\left\langle a_{T}\right\rangle_{s, c^{*}}-D^{*} \kappa}{1+\mathcal{L}_{\kappa}^{t} \kappa}\right] p(\kappa) d \kappa \\
& +\rho^{*} \int_{0}^{+\infty}\left[S_{d}^{0}-\mathcal{L}_{a_{T}}^{t}\left\langle a_{T}\right\rangle_{s, c^{*}}-D^{*}{ }^{*}-\mathcal{L}_{\kappa}^{t} S_{d}^{0} \kappa\right] p(\kappa) d \kappa .
\end{aligned}
$$

In Figs. 25d to 25f, the corrected model (in green solid line) is found to be in much better agreement with the DNS data on the fresh gases side compared to the proposed model in Eq. (35). For $L e=1$ cases, the models based on Eqs. (35) and (49) are nearly identical as in this case, the curvature Markstein lengths are much smaller.

\subsection{Summary of model parameters}

Table 5 presents an overview of the closure relations retained in the final model, involving a significant number of parameters corresponding to 14 constants. Some of these constants are directly taken from the ECFMLES combustion model [22]:

- Two constants for the model of curvature (Eq. 39, $\beta_{1}=4 / 3$ and $c_{1}=0.5$, are fixed to the same values as proposed by Richard et al. [22] and are assumed to be identical for other conditions.

- The model parameter $\alpha_{a_{T}}$ involved in the model for tangential strain rate (Eq. 42) plays the same role as in the ECFM and ECFM-LES combustion models [22]. In practical applications, this is the only parameter that is tuned to fit experimental results.

In the current analysis, some constants were added by introducing new models:

- In the model for $\left\langle\kappa^{2}\right\rangle_{s, c^{*}}$ (Eq. 40, the model parameter $\beta_{2}$ was introduced. This parameter was set equal to one for a correct agreement with DNS. Its dependency to thermodynamic and mixture properties should be investigated in future work.

- The model for $\left\langle S_{d}\right\rangle_{s, c^{*}}$ (Eq. 49) is based on the use of effective Markstein lengths $\mathcal{L}_{\kappa}^{t}$ and $\mathcal{L}_{a_{T}}^{t}$ (Eqs. 30 and 31 involving model parameters, which are:

- $\mathcal{L}_{K}^{l}$ and $\mathcal{L}_{a_{T}}^{l}$ which are the laminar strain and curvature Markstein lengths. These parameters are uniquely defined computing strained and curved laminar flames as proposed in this study.

- Parameters $a, b, \alpha, \beta, \mathcal{M}_{\kappa}^{\infty}$ and $\mathcal{M}_{a_{T}}^{\infty}$ are tuned to best fit the present DNS. As the proposed expression only depends on the Karlovitz number which is an adimensional quantity, it is expected that this expression should correctly describe other turbulent flames. This should be verified in future work involving a larger range of turbulent flames (different equivalence ratios, fuels, dilution rates, ... ).

- The retained model for tangential strain rate, LPF, (Eq. 47), involves an additional parameter, which is the cut-off length $\delta_{c}$. As shown in the supplementary material, the sensitivity to this parameter is weak and choosing $\delta_{c}=\delta_{L}$ allows to correctly recover the DNS strain. As for the effective Markstein length, future work should assess the validity of this model on different turbulent flames.

Finally, the chosen approach implies to choose a particular iso-surface $c^{*}$ in the reaction zone, which here corresponds to values between 0.5 and 0.9 . Thus, $c^{*}$ appears as a new model parameter, but it is expected that 
Table 5: Summary of the closures involved in the proposed model.

\begin{tabular}{|c|c|}
\hline \multicolumn{2}{|r|}{ Progress variable: $C=\mathcal{H}\left(c-c^{*}\right)$} \\
\hline Filtered equation: & $\frac{\partial \bar{\rho} \widetilde{C}}{\partial t}+\nabla \cdot(\overline{\rho \mathbf{u} C})=\left\langle\rho S_{d}\right\rangle_{s, c^{*}} \bar{\Sigma}^{*}$ \\
\hline \multirow[t]{5}{*}{ Closure of $\left\langle\rho S_{d}\right\rangle_{s, c^{*}}$} & $\begin{array}{l}\rho^{*} \int_{\kappa_{\min }}^{0}\left[\frac{S_{d}^{0}-\mathcal{L}_{a_{T}}^{t}\left\langle a_{T}\right\rangle_{s, c^{*}}-D^{*} \kappa}{1+\mathcal{L}_{\kappa}^{t} \kappa}\right] p(\kappa) d \kappa \\
+\rho^{*} \int_{0}^{+\infty}\left[S_{d}^{0}-\mathcal{L}_{a_{T}}^{t}\left\langle a_{T}\right\rangle_{s, c^{*}}-D^{*} \kappa-\mathcal{L}_{\kappa}^{t} S_{d}^{0} \kappa\right] p(\kappa) d \kappa\end{array}$ \\
\hline & submodels \\
\hline & $\frac{1}{\sqrt{2 \sigma^{2} \pi}} \exp \left(-\frac{\left(\kappa-\langle\kappa\rangle_{s, c^{*}}\right)^{2}}{2\left(\left\langle\kappa^{2}\right\rangle_{s, c^{*}}-\langle\kappa\rangle_{s, c^{*}}^{2}\right)}\right)$ \\
\hline & $\beta_{1} \frac{c_{1}-\bar{C}}{\bar{C}(1-\bar{C})}\left(\bar{\Sigma}^{*}-|\nabla \bar{C}|\right)$ \\
\hline & $\beta_{2} \frac{1}{\bar{C}^{2}(1-\bar{C})^{2}}\left(\bar{\Sigma}^{*}-|\nabla \bar{C}|\right)^{2}$ \\
\hline \multicolumn{2}{|r|}{ FSD: $\bar{\Sigma}^{*}=\overline{|\nabla C|}=\overline{|\nabla c| \delta\left(c-c^{*}\right)}$} \\
\hline Filtered equation: & $\begin{aligned} \frac{\partial \bar{\Sigma}^{*}}{\partial t}+\nabla \cdot\left(\langle\mathbf{u}\rangle_{s, c^{*}} \bar{\Sigma}^{*}\right)=\left\langle a_{T}\right\rangle_{s, c^{*}} \bar{\Sigma}^{*} & +\left\langle S_{d} \nabla \cdot \mathbf{n}\right\rangle_{s, c^{*}} \bar{\Sigma}^{*} \\
& -\nabla \cdot\left(\left\langle S_{d} \mathbf{n}\right\rangle_{s, c^{*}} \bar{\Sigma}^{*}\right)\end{aligned}$ \\
\hline Closure of $\left\langle S_{d} \kappa\right\rangle_{s, c^{*}}$ & $\int_{\kappa_{\min }}^{+\infty} \frac{S_{d^{0}}^{0} \kappa-\mathcal{L}_{a_{T}}^{t}\left\langle a_{T}\right\rangle_{s, c^{*}} \kappa-D^{*} \kappa^{2}}{1+\mathcal{L}_{\kappa}^{t} \kappa} p(\kappa) d \kappa$ \\
\hline Closure of $\left\langle a_{T}\right\rangle_{s, c^{*}}$ & $\alpha_{a_{T}} \frac{3}{\sqrt{55}} K a \frac{S_{L}^{0}}{\delta_{L}}\left[1-\exp \left(-\frac{3}{2} C_{k} \frac{1}{R e_{t}}\left(\frac{\pi l_{t}}{\delta_{c}}\right)^{4 / 3}\right)\right]^{1 / 2}$ \\
\hline
\end{tabular}

$\kappa_{\min }=\max \left(\frac{\varepsilon-1}{\mathcal{L}_{K}^{t}},-\frac{2}{\delta_{L}}\right)$. 
the model results would not change significantly when varying $c^{*}$ in this range.

\section{Conclusions}

A DNS database of freely propagating statistically planar turbulent $\mathrm{C}_{8} \mathrm{H}_{18}$-air premixed flames at different Karlovitz numbers was performed using simplified chemistry. The resulting cases are obtained at the same integral length scale but for different turbulent velocity fluctuations. The impact of turbulence on the flame is studied using a turbulence forcing method, to allow the flame to reach a statistically stationary state. In addition, a simulation using a more detailed chemical mechanism showed that the influence of the chemical scheme on the general behavior of the flame is moderate. Then, differential diffusion effects were systematically isolated by performing the simulations with both non-unity and unity Lewis numbers. The resulting analysis of the flame/turbulence interactions, from the flamelet to TRZ regimes, was then used to evaluate the modeling implications.

First, the flame surface and turbulent flame speed $S_{T}$ were found to show a bending with increasing Karlovitz number in line with previous studies [6-11]. A shown in previous studies, differential diffusion has a strong impact on the flame wrinkling of present DNS, which reaches higher values with $L e=1$ than with $L e>1$ for the same turbulence intensity, but also on the bending of $S_{T}$. The flame thickness is also influenced by turbulent intensity and Lewis number: turbulent flames with $L e>1$ are thickened over the whole flame, even in the reaction zone, while flames with $L e=1$ are thinner and mainly thickened in the preheat zone. Thus, theories considering a thickening only in the preheat zone seem well suited for unity Lewis number flames, but not for flames with $L e>1$. However, the reaction zone for these cases remains thin relatively to the preheat zone, and the mean conditional reaction rate of the progress variable remains close to that of a laminar flame, suggesting that the assumption of the TRZ regime remains valid.

The DNS analysis shows that $\left\langle S_{d} / S_{d}^{0}\right\rangle_{s, c^{*}}$ decreases on the fresh gas side (when $c^{*}$ decreases) while the flame surface density $\bar{\Sigma}^{*}$ increases so that the product $\left\langle S_{d} / S_{d}^{0}\right\rangle_{s, c *} \bar{\Sigma}^{*}$ remains constant, which is a consequence of the statistical stationarity of the flame. The modeling implication is that a fine grained FSD approach combined to the coherent flame model appears adequate to evaluate the flame speed, particularly if $c^{*}$ is chosen in the reactive region.
Then, the impact of turbulence on the displacement speed $S_{d}$ is explored through the response to stretch of an iso-surface of the progress variable at $c^{*}=0.8$. This value is retained as it corresponds to an iso-surface close to the peak of heat release rate showing the lowest perturbations of the iso-surface by turbulence. It also corresponds to the closest value of the stretch factor $I_{0}$ to unity, that is, a displacement speed close to that of the laminar flame. An analytical expression for $S_{d}$, Eq. (28), is then proposed based on strain and curvature. This expression considers a tangential diffusion term $-D \kappa$ as suggested by Peters, but present DNS results show that the sum of reaction and normal diffusion components $S_{r}+S_{n}$ cannot be assumed constant and equal to the laminar flame speed. For this reason, $S_{r}+S_{n}$ is modeled retaining a laminar flame speed expression devised for $S_{d}$ from asymptotic theories [76]. Laminar Markstein lengths are then replaced by effective strain and curvature Markstein lengths. Fitting these Markstein lengths on the DNS results, they are shown to decrease strongly with increasing Karlovitz number, in good agreement with previous studies which observed a decrease of the effective Lewis number with $K a$ [18, 39. 43]. The proposed expression of $S_{d}$ allows to reproduce correctly the response of $S_{d}$ to stretch at all the Karlovitz number considered, in spite of the strong assumptions and approximations it involves.

Closures for the adapted equations of the CFM model are then proposed, focusing on the displacement speed $\left\langle\rho S_{d}\right\rangle$, the stretch due to curvature $\left\langle S_{d} \kappa\right\rangle$, and the turbulent strain $\left\langle a_{t}\right\rangle$. Models for $\left\langle\rho S_{d}\right\rangle$ and $\left\langle S_{d} \kappa\right\rangle$, see Eq. (49) and (38), are obtained by integration of the proposed expression of $S_{d}$, Eq. (28), over curvature, using a presumed Gaussian PDF for curvature. This PDF is itself determined thanks to the mean curvature model of Rymer [48], and to a new model for $\left\langle\kappa^{2}\right\rangle$, Eq. 40. Models for $\left\langle\rho S_{d}\right\rangle$ and $\left\langle S_{d} \kappa\right\rangle$ are found in good agreement with the DNS, even at the largest Karlovitz numbers. At the same time, it is shown that these models are highly sensitive to the modelling of the effective strain and curvature Markstein lengths, which require therefore future research to reach good predictivity. Finally, a new closure for the turbulent strain rate $\left\langle a_{t}\right\rangle$ is proposed, Eq. (47), based on a simplification of Charlette's efficiency function. In this model called LPF (Low Pass Filter), the efficiency of vortices is considered equal to unity down to a cut-off length scale $\delta_{c}$, then equal to zero below this scale. Choosing the laminar flame thickness for $\delta_{c}$ leads to a better agreement with DNS results compared to previous models.

The a posteriori validation of the proposed closures will be the subject of future work. 


\section{Acknowledgements}

We kindly acknowledge Dr. Luis Miguel Segui Troth for generous support on the turbulent forcing technique (during his post-doc at CERFACS) and Dr. Corentin Lapeyre (CERFACS) for providing the ARC mechanism. We warmly thank the reviewers for their constructive comments which greatly helped to improve the quality of the paper.

\section{Funding}

This work was supported by a grant overseen by the French National Research Agency (ANR) to the ANR-15-CE22-0014 MACDIL project coordinated by IFPEN.

This work was also granted access to the HPC resources of CINES under allocation no. A0062B10763 from the GENCI (Grand Equipement National de Calcul Intensif) eDARI program and to resources during the 17th Call of PRACE Project Access (Pra102).

\section{Appendix A. Discussions on the turbulence forcing method}

The turbulence forcing method described in section 3.2 can introduce some undesirable effects on the flame front, especially on the flame stretch and the flame displacement speed. An additional case $\mathrm{B}_{\mathrm{FG}}$ corresponding to case $\mathrm{B}$ conditions is performed with the forcing term $\mathbf{f}$ in the momentum equation weighted with the progress variable following Eq. A.1. This alternative forcing method allows to force the velocity field only in the unburned gases.

$$
\mathbf{f}_{F G}=\left(1-\tanh \left(\frac{c}{c_{F G}}\right)\right) \mathbf{f},
$$

where $c_{F G}$ is a limit value to control the decrease of the forcing term $\mathbf{f}$ relatively to the flame. This limit is set here to 0.01 , which means that forcing is essentially suppressed for $c>0.05$.

The simulation parameters of both cases $\mathrm{B}$ and $\mathrm{B}_{\mathrm{FG}}$ are reported in Table A.6.

Figure A.26 presents the temporal evolution of some variables of interest. In Fig. A.26a the flame wrinkling $\Xi$ of case $\mathrm{B}_{\mathrm{FG}}$ is smaller than for case $\mathrm{B}$, by a reduction of approximately $30 \%$. This is to be related to the tangential strain rate $\left\langle a_{T}\right\rangle_{s}$ in Fig. A.26b which is also reduced by $30 \%$. Furthermore, the heat release rate presented in Fig. A.26d shows a similar decrease in case $\mathrm{B}_{\mathrm{FG}}$ compared to case $\mathrm{B}$. However, the evolution
Table A.6: The DNS set-up for the simulations $\mathrm{B}$ and $\mathrm{B}_{\mathrm{FG}}$ of the interaction between a planar flame and forced homogeneous isotropic turbulence.

\begin{tabular}{|c|c|c|c|c|c|c|c|}
\hline \multicolumn{2}{|c|}{ CaseLe $_{\text {Fuel }}$} & $\mathbf{u}^{\prime} / \mathbf{S}_{\mathbf{L}}^{\mathbf{0}}$ & $\mathbf{l}_{\mathbf{t}} / \delta_{\mathbf{L}}$ & $\mathbf{R e}_{t}$ & $\mathbf{K a}$ & Da & $\eta_{\mathbf{k}} / \boldsymbol{\Delta}_{\mathbf{x}}$ \\
\hline B & 2.9 & 12.5 & 4.3 & 489.0 & 21.4 & 0.3 & 0.6 \\
\hline $\mathrm{B}_{\mathrm{FG}}$ & 2.9 & 11.7 & 4.1 & 427.0 & 19.8 & 0.4 & 0.7 \\
\hline
\end{tabular}

of both the stretch factor $I_{0}$ and the displacement speed shown in Fig. A.26c present the same orders of magnitude and similar temporal evolution for both cases $\mathrm{B}$ and $\mathrm{B}_{\mathrm{FG}}$. Thus, the decrease of the heat release, when the turbulence is only forced in the fresh gases, is explained by the decrease of the turbulent flame surface. It seems that the forcing method does not influence the inner structure of the flame and has mainly an effect on the surface through an increase of the tangential strain rate.

The evolution of the conditional mean of the displacement speed conditioned with the curvature for case $\mathrm{B}_{\mathrm{FG}}$ is compared to case $\mathrm{B}$ in Fig. A.27 The overall evolution of $\left\langle S_{d}^{*}\right\rangle_{K}$ is shown to be similar with slightly smaller slopes for case $\mathrm{B}_{\mathrm{FG}}$ which can be explained by the moderate decrease of the turbulence intensity in the flame front when forcing only in the fresh gases.

Figure A.28 presents the evolution of $\langle\kappa\rangle_{s, c^{*}},\left\langle\kappa^{2}\right\rangle_{s, c^{*}}$, $\left\langle S_{d} \kappa\right\rangle_{s, c^{*}}$ and $\left\langle S_{d}\right\rangle_{s, c^{*}}$ for cases $\mathrm{B}$ and $\mathrm{B}_{\mathrm{FG}}$ as functions of $\widetilde{C}$. All these quantities remain similar between $\mathrm{B}$ and $\mathrm{B}_{\mathrm{FG}}$, with a tendency to smoother profiles (due to slightly less intense turbulence) and decrease $\left\langle S_{d} \kappa\right\rangle_{s, c^{*}}$ and flatten $\left\langle S_{d}\right\rangle_{s, c^{*}}$ for case $\mathrm{B}_{\mathrm{FG}}$ compared to case $\mathrm{B}$.

From this additional case it can be concluded that the turbulence forcing throughout the flame front does not alter significantly the statistics.

\section{Appendix B. Evaluation of Markstein lengths using stretched flames}

Several techniques reported in [46] have been used to estimate a Markstein length. In this study, two effective Markstein lengths need to be estimated, one for the effect of tangential strain rate and one for the effect of curvature on the flame. Thus, two techniques allowing to isolate both effects were chosen: a counter-flow premixed flame for the tangential strain and a spherical flame for the effect of curvature. 


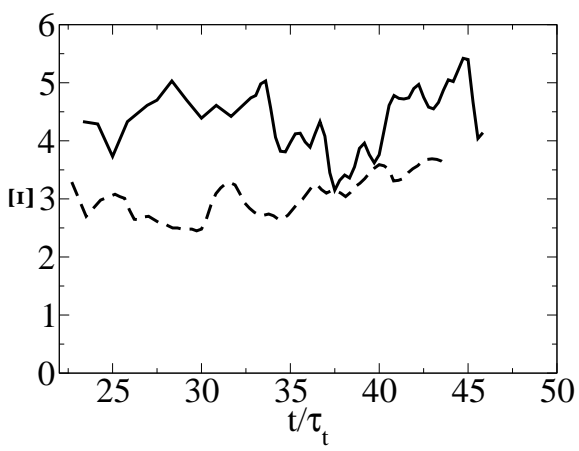

(a)

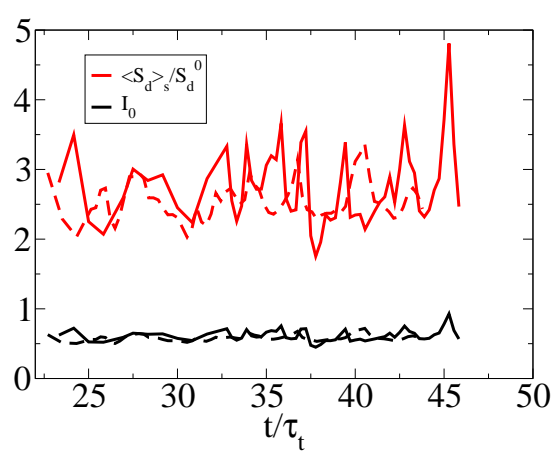

(c)

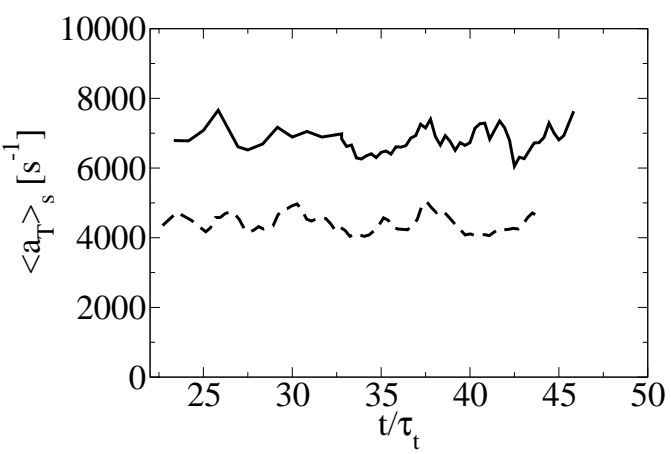

(b)

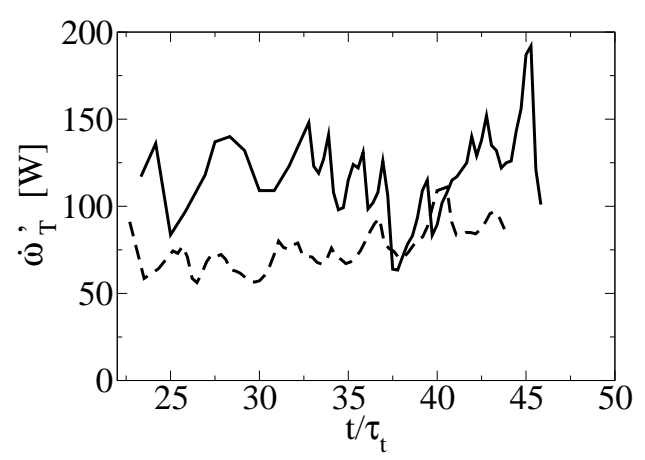

(d)

Figure A.26: Comparison of the overall results by forcing the turbulence in the whole domain (case B) in solid lines or in the fresh gases only (case $\mathrm{B}_{\mathrm{FG}}$ ) in dashed lines: (a) the temporal evolution of the generalized flame surface, $(\mathrm{b})$ the temporal evolution of the generalized tangential strain rate, (c) the temporal evolution of the displacement speed in red and of the stretch factor $I_{0}$ in black and (d) the temporal evolution of the heat release.

Using the open-source solver Cantera, a counter-flow iso-octane/air premixed flame is computed as described by Darabiha et al. in [89] and illustrated in Fig. B.29a Two flames are simulated, one with $L_{e k} \neq 1$ for each species and one with unity Lewis numbers using the two-step mechanism presented in Section 3.1. The flame stretch rate in this configuration is only due to tangential strain rate, i.e $K=a_{T}$. As presented by Van Oijen [90], the stretch is computed as follows:

$$
K=\frac{1}{\rho} \frac{d \rho u}{d x} .
$$

$S_{r}^{*}+S_{n}^{*}$ at $c=c^{*}=0.8$ is extracted and plotted as a function of $K^{*}$ in Fig. B.30 The dashed line in Fig. B.30, represents the linear relationship: $S_{r}^{*}+S_{n}^{*}=S_{d}^{0}-\mathcal{L}_{a_{T}} K^{*}$, where $\mathcal{L}_{a_{T}}$ is the Markstein length with $a_{T}^{*}$. The latter is fitted to best reproduce the evolution of $S_{r}^{*}+S_{n}^{*}$ for low stretch.

Using AVBP-code, one $8^{\text {th }}$ of a freely propagating spherical iso-octane/air premixed laminar flame is simu- lated (see Fig. B.29b). As for the spherical flames, both $L_{e k} \neq 1$ and $L_{e k}=1$ flames are simulated. With this configuration, the flame stretch is computed as follows:

$$
K=\frac{1}{\mathcal{A}} \frac{d \mathcal{A}}{d t}=\frac{2}{R_{b}} \frac{d R_{b}}{d t},
$$

where $\mathcal{A}$ is the flame surface, $R_{b}$ is the radius of the spherical flame taken in the burnt gases.

Figure B.31 displays the temporal evolution of the stretch rate $K^{*}$, the tangential strain rate $a_{T}^{*}$ and the stretch rate due to curvature $\left(S_{d} K\right)^{*}$ for $c=c^{*}=0.8$. This figure shows that $\left(S_{d} \kappa\right)^{*}$ is dominant, representing about $85 \%$ of the total stretch rate at $t=3 \mathrm{~ms}$.

To evaluate $\mathcal{L}_{\kappa}^{l}$, the quantity $S_{r}^{*}+S_{n}^{*}+\mathcal{L}_{a_{T}}^{l} a_{T}^{*}$ is extracted from the DNS of the spherical flame, where $\mathcal{L}_{a_{T}}^{l}$ is the Markstein length estimated with the counter-flow premixed flame. It is plotted as a function of $\left(S_{d} \kappa\right)^{*}$ in Fig. B.32 $\mathcal{L}_{K}^{l}$ is deduced as the best fit of this function by the linear relation $S_{r}^{*}+S_{n}^{*}+\mathcal{L}_{a_{T}}^{l} a_{T}^{*}=S_{d}^{0}-\mathcal{L}_{K}^{l}\left(S_{d} \kappa\right)^{*}$. This fit corresponds to the dashed line in Fig. B.32 


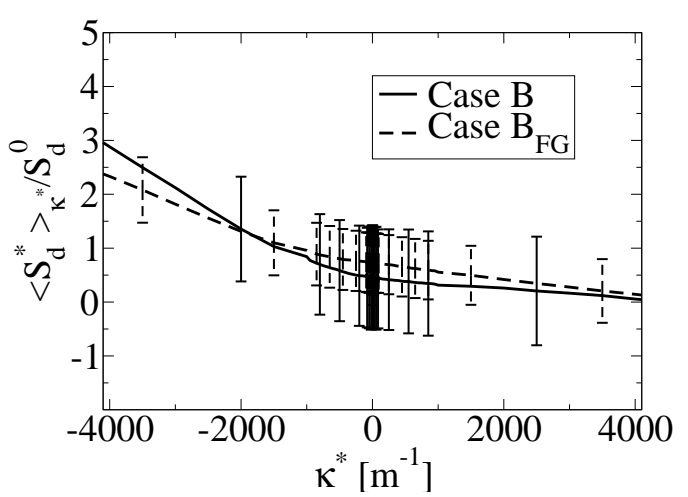

(a)

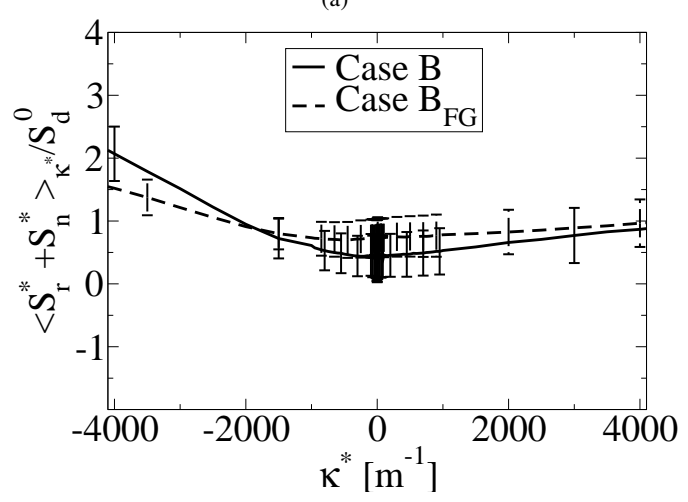

(b)

Figure A.27: Comparison of the responses of the displacement speed to curvature by forcing the turbulence in the whole domain (case B) or in the fresh gases only (case $\mathrm{B}_{\mathrm{FG}}$ ): a the evolution of the conditional mean displacement speed, $\left\langle S_{d}^{*}\right\rangle_{\kappa}$, normalized with the laminar flame speed at $c=c^{*}$ as a function of the curvature $\kappa, b$ b the evolution of $\left\langle S_{r}^{*}+S_{n}^{*}\right\rangle_{K}$ normalized with the laminar flame speed at $c=c^{*}$ as a function of the curvature $\kappa$. The solid lines are used for the results from case $\mathrm{B}$ and the dashed lines represents the results from case $\mathrm{B}_{\mathrm{FG}}$. The error bars represent the standard deviation.

\section{Appendix C. Discussion on chemical mechanisms}

The response to strain rate of the 2-steps mechanism is compared to the analytically reduced mechanism ISOOCT18. Table C.7 shows the flame characteristics for each mechanism.

Both mechanisms predict very similar laminar flame speeds with less than $2 \%$ difference relatively to ISOOCT18 mechanism. Note that the laminar flame thicknesses $\delta_{L}$ computed for each mechanism differ significantly, by approximately $30 \%$ relatively to ISOOCT18 mechanism.

Figure C.33 shows the evolution of $S_{r}^{*}+S_{n}^{*}$ as a function of the tangential strain rate $a_{T}^{*}$ normalized with the laminar flame time $\tau_{F}=\delta_{L} / S_{L}^{0}$ extracted from counterflow premixed flame computed with Cantera using the 2-steps mechanism, the reduced analytical mechanism ISOOCT18 and the reduced SPK mechanisms in black,
Table C.7: Flame characteristics using the 2-steps mechanism and the reduced analytical mechanism ISOOCT18.

\begin{tabular}{lccc} 
Mechanism & $\mathbf{S}_{\mathbf{L}}^{\mathbf{0}}[\mathbf{m} / \mathbf{s}]$ & $\delta_{\mathbf{L}}[\mu \mathbf{m}]$ & $\tau_{\mathbf{F}}[\mathbf{m s}]$ \\
\hline 2-steps & 0.366 & 385 & 1.11 \\
ISOOCT18 & 0.360 & 560 & 1.56 \\
\hline
\end{tabular}

red and blue solid lines, respectively.

In this figure, the flames using SPK and ISOOCT18 mechanisms present the same evolution for $S_{r}^{*}+S_{n}^{*}$ confirming that ISOOCT18 mechanism is well suited to capture the effect of preferential diffusion. The evolution of $S_{r}^{*}+S_{n}^{*}$ with $a_{T}^{*} \tau_{F}$ using the 2-steps mechanism is similar to those using the reduced mechanisms. However, even if the flame using 2-steps mechanism also presents a decreasing evolution of $S_{r}^{*}+S_{n}^{*}$ with increasing $a_{T}^{*} \tau_{F}$, its slope (corresponding to Markstein number) is slightly smaller than the flame using ISOOCT18 mechanism. Indeed, the Markstein numbers $\mathcal{M}_{a_{T}}^{l}$ deduced from Fig. C.33 are 1.6 and 1.2 for ISOOCT18 and 2-steps mechanisms, respectively.

As shown in subsection 4.5 and as supported by previous study [39], the response to strain rate also depends on turbulence. Thus, similarly to case B a turbulent Markstein number $\mathcal{M}_{a_{T}}^{t}$ is computed from DNS for case B-ARC. Figure C.34 compares Markstein numbers of the flames simulated with ISOOCT18 and 2-step mechanisms in plain triangle and empty circle symbols, respectively.

In this figure, the Markstein number of the flame computed with the ISOOCT18 mechanism decreases with increasing Karlovitz number with a steeper slope than with the 2-steps mechanism (from 1.63 for $K a=0$ to 0.37 for $K a=21.4$, to be compared to 1.19 and 0.60 respectively with the two step-mechanism). Consequently, the expression in Eq. (30) fitted on the 2-steps mechanism could slightly differ with ISOOCT18 mechanism. However, these results show that flames simulated with ISOOCT18 and 2-steps mechanisms present a qualitatively similar response to strain rate. The differences observed are acceptable but confirm that further investigations on turbulent flame involving detailed chemical mechanisms are needed to better understand and model the strain rate response. 


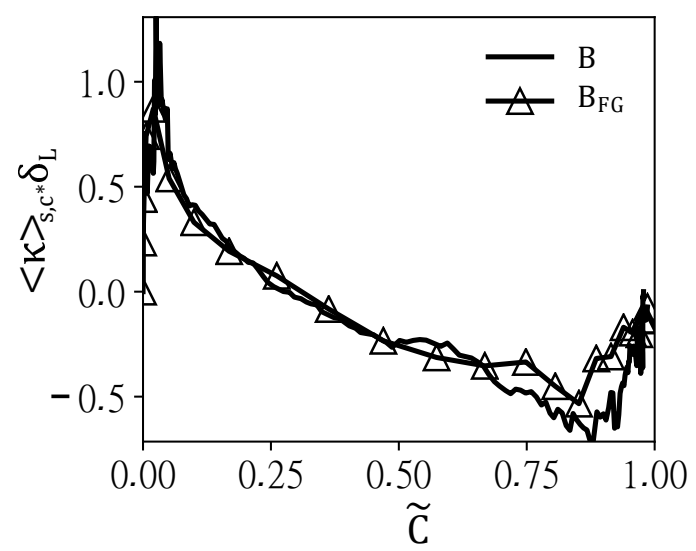

(a)

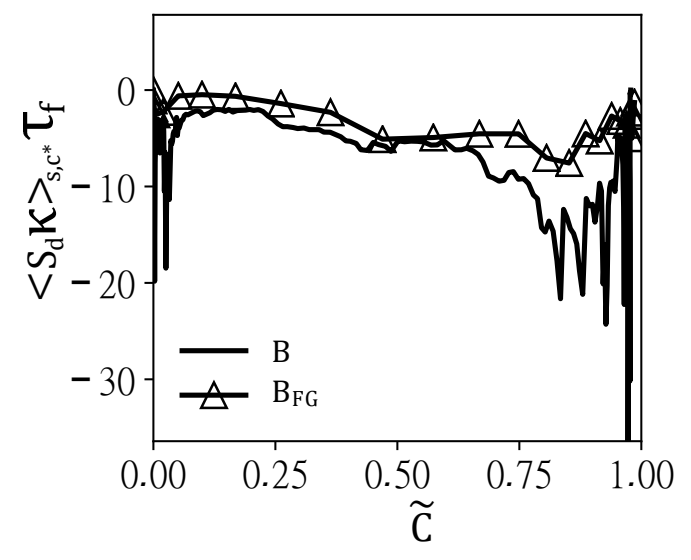

(c)

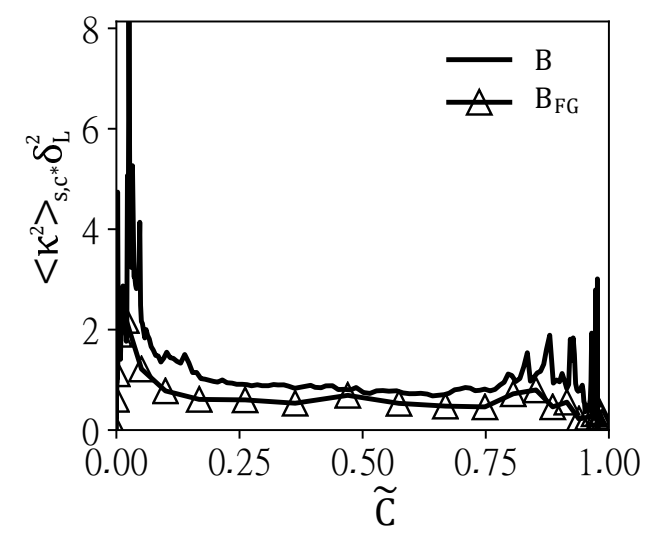

(b)

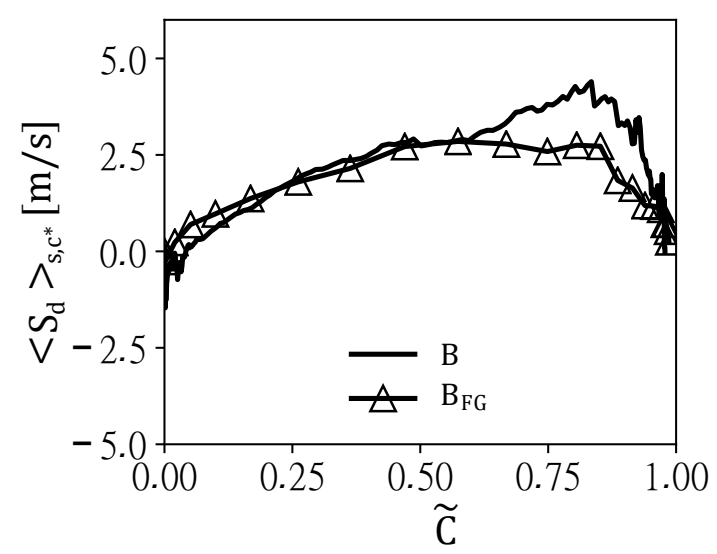

(d)

Figure A.28: Comparison of the profiles by forcing the turbulence in the whole domain (case B) or in the fresh gases only (case $\mathrm{B}_{\mathrm{FG}}$ ): a the evolution of $\langle\kappa\rangle_{s, c^{*}}$, b the evolution of $\left\langle\kappa^{2}\right\rangle_{s, c^{*}}$, c c the evolution of $\left\langle S_{d} \kappa\right\rangle_{s, c^{*}}$, and (d) the evolution of $\left\langle S_{d}\right\rangle_{s, c^{*}}$.

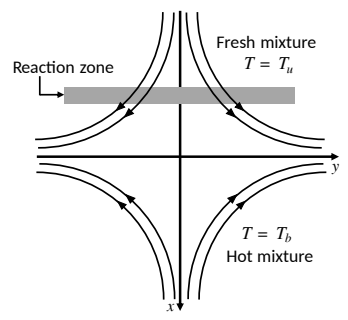

(a) Schematic view of a counterflow premixed flame.

Figure B.29: Schematic view of laminar stretched ames.

\section{Appendix D. Post-processing methodology}

The analysis of the flame consists in extracting the mean part and the fluctuating part of quantities of interest at each time step. In this study we discretize the simulation domain along the propagation axis, as illustrated in Fig. D.35 The flames being statistically uniform in the $\mathrm{y}$ and $\mathrm{z}$ directions, a spatial averaging of $c$ (or $C$ ) in each slice of thickness $\delta_{x}$ is performed allowing to get profiles of $\bar{c}$ (respectively $\bar{C}$ ) and $\widetilde{c}$ (respectively $\widetilde{C}$ ) against the propagation axis $x$. The value of $\delta_{x}$ is chosen as $\delta_{x}=2 \Delta_{x}$, where $\Delta_{x}$ is the cell size of the DNS.

Then, from the extraction of the iso-surface $c=c^{*}$, the surface averaging operation, defined in Eq. (D.1), is applied to each variable of interest. From the profiles of $\widetilde{c}(t)(\widetilde{C})$ and of $\langle Q(t)\rangle_{s, c^{*}}$ versus $x$, the profiles of $\langle Q(t)\rangle_{s, c^{*}}$ against $\widetilde{c}(t)$ (or $\left.\widetilde{C}(t)\right)$ are deduced.

$$
\langle Q(t)\rangle_{s, c^{*}}=\frac{\int_{\mathcal{V}_{x}} Q(\mathbf{x}, t) \mathrm{d} \mathcal{A}^{*}}{\mathcal{A}^{*}(t)},
$$

where $Q$ is a general quantity, $\mathcal{V}_{x}$ is the volume of the slice at position $x$ and $\mathcal{A}^{*}$ is the area of flame surface contained in the slice.

Finally, the profiles of $\langle Q(t)\rangle_{s, c^{*}}$ versus $\widetilde{c}(t)$ (or $\widetilde{C}(t)$ ) are temporally averaged on the quasi-steady state phase 


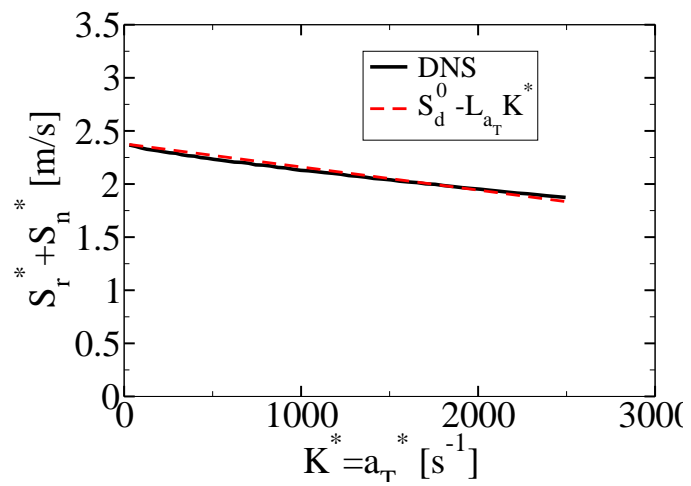

(a)

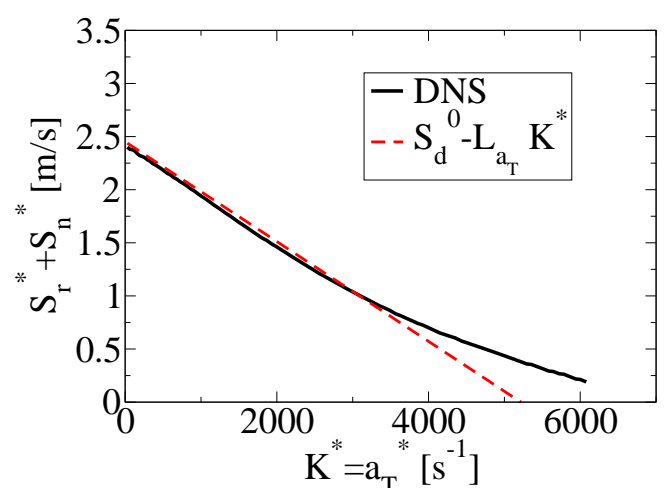

(b)

Figure B.30: Evolution of the sum $S_{r}^{*}+S_{n}^{*}$ as a function of stretch $K^{*}$ for counter-flow premixed flames for (a) unity Lewis numbers case and (b) non-unity Lewis numbers case.

identified in Section 4

\section{Appendix E. Katragadda's model for stretch due to curvature}

Katragadda et al. [38] proposed a model for the stretch due to curvature in the context of generalized FSD transport equation. This model was developed in the context of the TRZ regime, and is given below:

$$
\begin{aligned}
& \left\langle S_{d} \kappa\right\rangle_{s} \bar{\Sigma}=-\beta_{1} S_{L}^{0}\left(1-{\overline{\langle\mathbf{N}\rangle_{s}}}_{\overline{\langle\mathbf{N}}\rangle_{s}}\right)\left(\bar{c}-c^{*}\right) f(\bar{c}, \widetilde{c}) \bar{\Sigma} \\
& -D_{e f f} \beta_{H}\left(1-\overline{\langle\mathbf{N}\rangle}_{s}{\overline{\langle\mathbf{N}\rangle_{s}}}_{)^{2}} \frac{1}{\bar{c}^{2}(1-\bar{c})^{2}} \bar{\Sigma},\right.
\end{aligned}
$$

where $\overline{\langle\mathbf{N}}_{s}=-\nabla \bar{c} / \bar{\Sigma}$ is the surface averaged normal vector, $D_{\text {eff }}$ is an effective diffusivity which is expected to approach the mass diffusivity for low $D a$ combustion, and $\beta_{1}, c^{*}, f(\bar{c}, \widetilde{c})$ and $\beta_{H}$ are model parameters defined

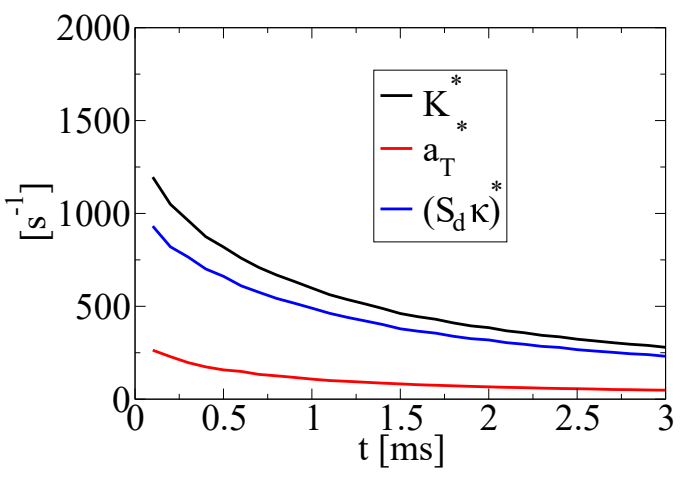

(a)

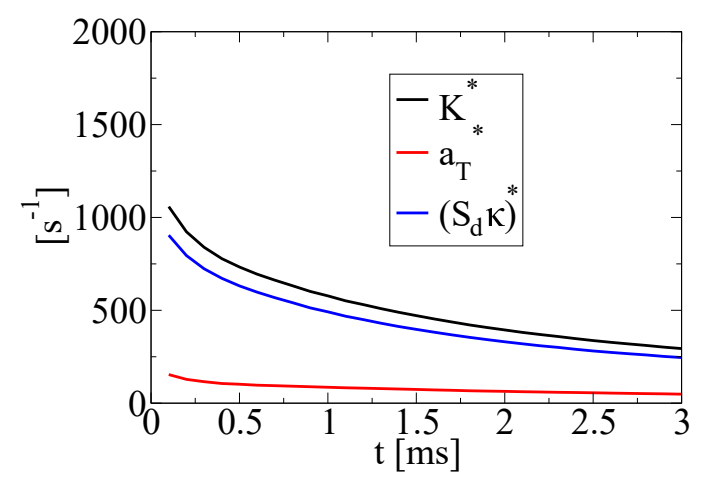

(b)

Figure B.31: Temporal evolution of the stretch rate $K^{*}$, the tangential strain rate $a_{T}^{*}$ and the stretch due to curvature $\left(S_{d} \kappa\right)^{*}$ for spherical premixed flames a for unity Lewis numbers case and (b) non-unity Lewis numbers case.

as follows:

$$
\beta_{1}=\left(7.24 L e^{-0.68}\right)(1+K a)^{-0.25}
$$

$$
c^{*}=\frac{1.27 \exp (-0.77 L e)}{\operatorname{erf}\left[(1+K a)^{1.1} / 4.85\right]}\left[1+\frac{f_{2}\left(R e_{t}\right)-1.0}{\left[1.0+\exp \left(-5.0\left(K a_{t h}-1.9\right)\right)\right]^{5}}\right],
$$

$$
f_{2}\left(R e_{t}\right)=0.49 \frac{R e_{t}^{0.41}+0.69}{0.46 R e_{t}^{0.46}+0.56}
$$

$$
f(\bar{c}, \widetilde{c})=1.0-\frac{\exp (-9.0(1-\widetilde{c}))}{\bar{c}(1.0-\bar{c})^{m}},
$$

$$
m=1.56 \frac{\exp (-0.24 L e)}{\operatorname{erf}[(1.0+K a) / 1.5]},
$$

$$
\beta_{H}=2.24 L e^{-0.85} \frac{0.5}{1+\exp \left[-\left(R e_{t}-20\right)\right]} \text {. }
$$




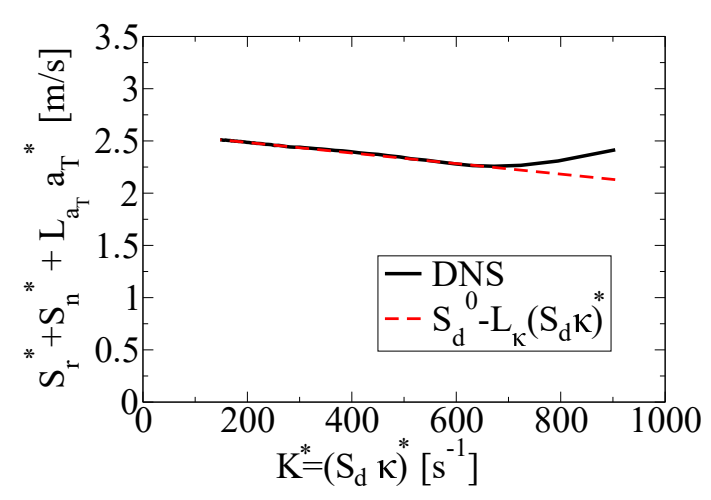

(a)

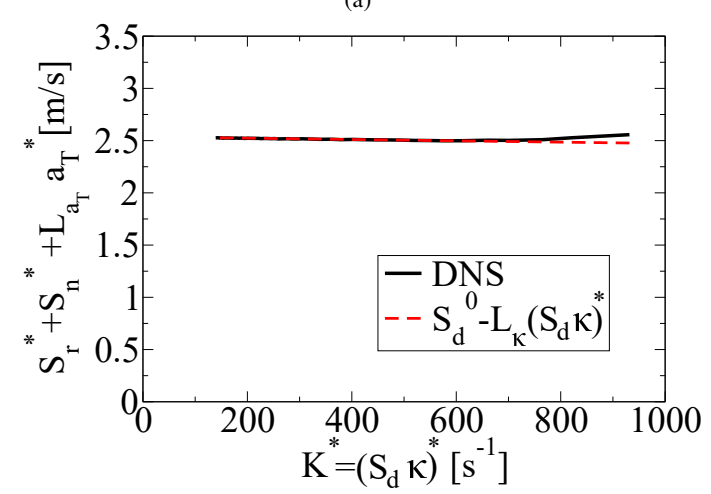

(b)

Figure B.32: Evolution of $S_{r}^{*}+S_{n}^{*}+\mathcal{L}_{a_{T}}^{l} a_{T}^{*}$ as a function of stretch $K^{*}=\left(S_{d}\right)^{*}$ for spherical premixed flames for a non-unity Lewis numbers case and $(\mathrm{b}$ for unity Lewis number case.

\section{References}

[1] N. Peters, The turbulent burning velocity for large-scale and small-scale turbulence, J. Fluid Mech. 384 (1999) 107-132.

[2] C. Mounaïm-Rousselle, L. Landry, F. Halter, F. Foucher, Experimental characteristics of turbulent premixed flame in a boosted spark-ignition engine, Proc. Combust. Inst. 34 (2) (2013) 29412949.

[3] A. Lipatnikov, J. Chomiak, Molecular transport effects on turbulent flame propagation and structure, Prog. Energy Combust. Sci. 31 (1) (2005) 1-73.

[4] S. Yang, A. Saha, W. Liang, F. Wu, C. K. Law, Extreme role of preferential diffusion in turbulent flame propagation, Combust. Flame 188 (2018) 498-504.

[5] P. Venkateswaran, A. Marshall, D. H. Shin, D. Noble, J. Seitzman, T. Lieuwen, Measurements and analysis of turbulent consumption speeds of h2/co mixtures, Combust. Flame 158 (8) (2011) 1602-1614.

[6] G. Nivarti, S. Cant, Direct numerical simulation of the bending effect in turbulent premixed flames, Proc. Combust. Inst. 36 (2) (2017) 1903-1910.

[7] G. V. Nivarti, R. S. Cant, Stretch rate and displacement speed correlations for increasingly-turbulent premixed flames, Flow Turbul. Combust. 102 (4) (2019) 957-971.

[8] T. M. Wabel, A. W. Skiba, J. F. Driscoll, Turbulent burning velocity measurements: Extended to extreme levels of turbulence, Proc. Combust. Inst. 36 (2) (2017) 1801-1808.

[9] Ö. L. Gülder, G. J. Smallwood, Flame surface densities in pre-

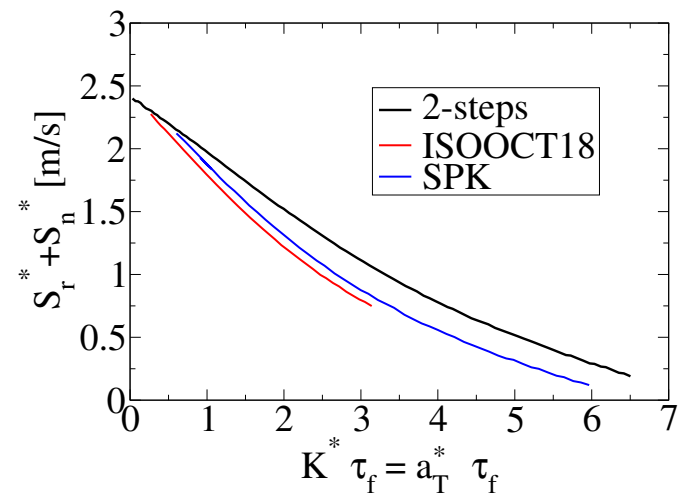

Figure C.33: Evolution of the sum $S_{r}^{*}+S_{n}^{*}$ as a function of stretch $K^{*}$ normalized with laminar flame time $\tau_{F}=\delta_{L} / S_{L}^{0}$ for counterflow premixed flame using SPK mechanism (blue), ISOOCT18 mechanism (red) and 2-steps mechanism (black).

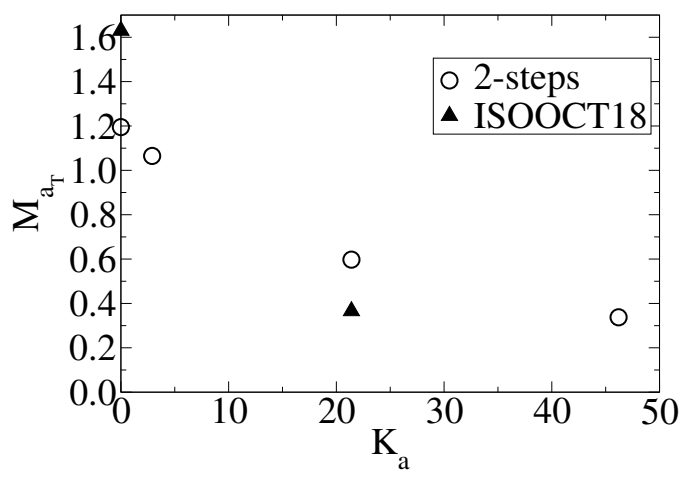

Figure C.34: Evolution of effective Markstein numbers with $K a$. The values computed from stretched laminar flame and from the optimization method are shown with plain triangle and empty circle symbols for ISOOCT18 and 2-steps mechanisms, respectively.

mixed combsution at medium to high turbulence intensities, Combust. Sci. Technol. 179 (1-2) (2007) 191-206.

[10] Ö. L. Gülder, Contribution of small scale turbulence to burning velocity of flamelets in the thin reaction zone regime, Proc. Combust. Inst. 31 (1) (2007) 1369-1375.

[11] U. Ahmed, N. Chakraborty, M. Klein, Insights into the bending effect in premixed turbulent combustion using the flame surface density transport, Combust. Sci. Technol. 191 (5-6) (2019) 898920 .

[12] H. Kido, M. Nakahara, K. Nakashima, J. Hashimoto, Influence of local flame displacement velocity on turbulent burning velocity, Proc. Combust. Inst. 29 (2) (2002) 1855-1861.

[13] V. Karpov, E. Severin, Effects of molecular-transport coefficients on the rate of turbulent combustion, Combust. Explos. Shock Waves 16 (1) (1980) 41-46.

[14] D. Bradley, M. Haq, R. Hicks, T. Kitagawa, M. Lawes, C. Sheppard, R. Woolley, Turbulent burning velocity, burned gas distribution, and associated flame surface definition, Combust. Flame 133 (4) (2003) 415-430.

[15] G. Nivarti, R. Cant, S. Hochgreb, Reconciling turbulent burning velocity with flame surface area in small-scale turbulence, $\mathrm{J}$. Fluid Mech. 858.

[16] F. T. Yuen, Ö. L. Gülder, Turbulent premixed flame front dynamics and implications for limits of flamelet hypothesis, Proc. 


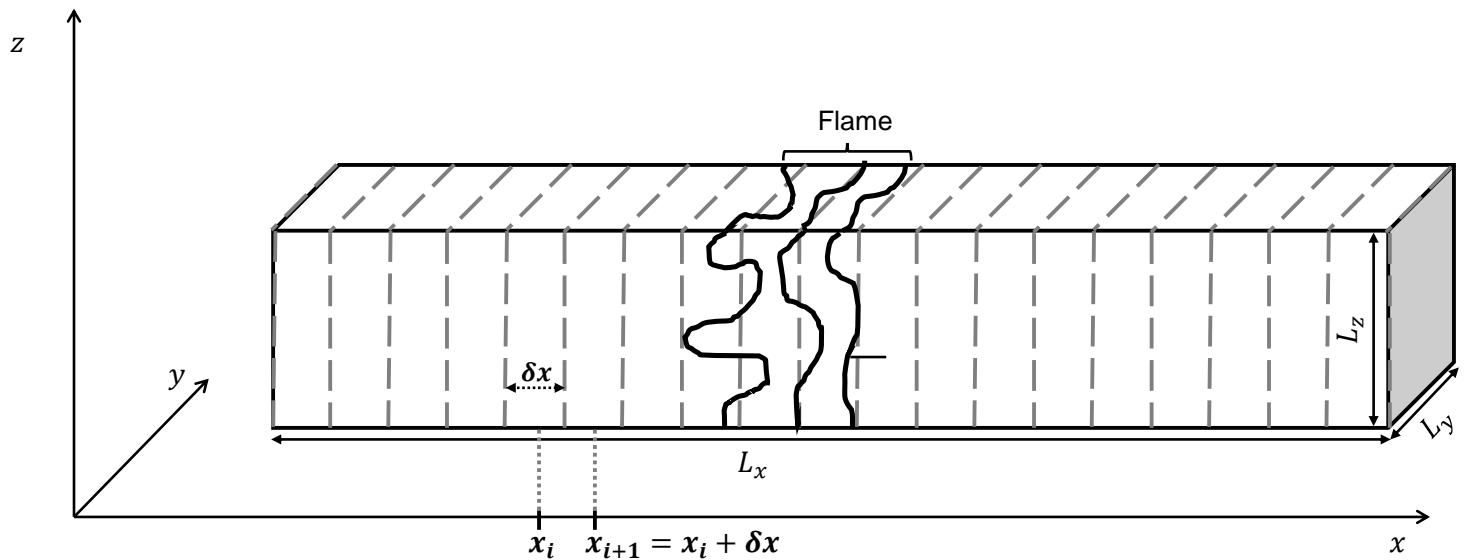

Figure D.35: Schematic view of the post-processing method.

Combust. Inst. 34 (1) (2013) 1393-1400.

[17] A. Poludnenko, E. Oran, The interaction of high-speed turbulence with flames: Turbulent flame speed, Combust. Flame 158 (2) (2011) 301-326.

[18] B. Savard, G. Blanquart, Broken reaction zone and differential diffusion effects in high karlovitz n-c7h16 premixed turbulent flames, Combust. Flame 162 (5) (2015) 2020-2033.

[19] N. Peters, Laminar flamelet concepts in turbulent combustion, Symp. (Int.) Combust. 21 (1) (1988) 1231-1250.

[20] O. Colin, F. Ducros, D. Veynante, T. Poinsot, A thickened flame model for large eddy simulations of turbulent premixed combustion, Phys. Fluids 12 (7) (2000) 1843-1863.

[21] H. Pitsch, A consistent level set formulation for large-eddy simulation of premixed turbulent combustion, Combust. Flame 143 (4) (2005) 587-598.

[22] S. Richard, O. Colin, O. Vermorel, A. Benkenida, C. Angelberger, D. Veynante, Towards large eddy simulation of combustion in spark ignition engines, Proc. Combust. Inst. 31 (2) (2007) 3059-3066.

[23] O. Colin, A. Benkenida, C. Angelberger, 3d modeling of mixing, ignition and combustion phenomena in highly stratified gasoline engines, Oil Gas Sci. Technol. 58 (1) (2003) 47-62.

[24] E. Hawkes, R. Cant, A flame surface density approach to largeeddy simulation of premixed turbulent combustion, Proc. Combust. Inst. 28 (1) (2000) 51-58.

[25] M. Boger, D. Veynante, H. Boughanem, A. Trouvé, Direct numerical simulation analysis of flame surface density concept for large eddy simulation of turbulent premixed combustion, Symp. (Int.) Combust. 27 (1) (1998) 917-925.

[26] J. Van Oijen, R. Bastiaans, G. Groot, L. De Goey, Direct numerical simulations of premixed turbulent flames with reduced chemistry: Validation and flamelet analysis, Flow Turbul. Combust. 75 (1-4) (2005) 67-84.

[27] H. L. Dave, S. Chaudhuri, Evolution of local flame displacement speeds in turbulence, J. Fluid Mech. 884 (1) (2020) A46.

[28] G. K. Giannakopoulos, A. Gatzoulis, C. E. Frouzakis, M. Matalon, A. G. Tomboulides, Consistent definitions of flame displacement speed and markstein length for premixed flame propagation, Combust. Flame 162 (4) (2015) 1249-1264.

[29] R. Yu, T. Nilsson, G. Brethouwer, N. Chakraborty, A. Lipatnikov, Assessment of an evolution equation for the displacement speed of a constant-density reactive scalar field, Flow Turbul. Combust. (2020) 1-20.

[30] E. R. Hawkes, J. H. Chen, Evaluation of models for flame stretch due to curvature in the thin reaction zones regime, Proc. Combust. Inst. 30 (1) (2005) 647-655.

[31] N. Chakraborty, R. S. Cant, Influence of Lewis number on curvature effects in turbulent premixed flame propagation in the thin reaction zones regime, Phys. Fluids 17 (10) (2005) 105105.

[32] N. Chakraborty, R. Cant, Influence of lewis number on strain rate effects in turbulent premixed flame propagation, Int. J. Heat Mass Transf. 49 (13-14) (2006) 2158-2172.

[33] N. Chakraborty, R. Cant, Effects of lewis number on flame surface density transport in turbulent premixed combustion, Combust. Flame 158 (9) (2011) 1768-1787.

[34] I. Han, K. Y. Huh, Effects of the karlovitz number on the evolution of the flame surface density in turbulent premixed flames, Proc. Combust. Inst. 32 (1) (2009) 1419-1425.

[35] R. Sankaran, E. R. Hawkes, C. S. Yoo, J. H. Chen, Response of flame thickness and propagation speed under intense turbulence in spatially developing lean premixed methane-air jet flames, Combust. Flame 162 (9) (2015) 3294-3306.

[36] H. Wang, E. R. Hawkes, J. H. Chen, B. Zhou, Z. Li, M. Aldén, Direct numerical simulations of a high karlovitz number laboratory premixed jet flame - an analysis of flame stretch and flame thickening, J. Fluid Mech. 815 (2017) 511-536.

[37] H. Wang, E. R. Hawkes, J. H. Chen, Turbulence-flame interactions in dns of a laboratory high karlovitz premixed turbulent jet flame, Phys. Fluids 28 (9) (2016) 095107.

[38] M. Katragadda, S. P. Malkeson, N. Chakraborty, Modelling of the curvature term in the flame surface density transport equation: a direct numerical simulations based analysis, Int. J. Spray Combust. Dyn. 6 (2) (2014) 163-198.

[39] B. Savard, G. Blanquart, An a priori model for the effective species lewis numbers in premixed turbulent flames, Combust. Flame 161 (6) (2014) 1547-1557.

[40] B. Savard, B. Bobbitt, G. Blanquart, Structure of a high karlovitz n-c7h16 premixed turbulent flame, Proc. Combust. Inst. 35 (2) (2015) 1377-1384.

[41] B. Savard, G. Blanquart, Effects of dissipation rate and diffusion rate of the progress variable on local fuel burning rate in premixed turbulent flames, Combust. Flame 180 (2017) 77-87.

[42] S. Lapointe, B. Savard, G. Blanquart, Differential diffusion effects, distributed burning, and local extinctions in high karlovitz premixed flames, Combust. Flame 162 (9) (2015) 3341-3355.

[43] S. Lapointe, G. Blanquart, A priori filtered chemical source term modeling for les of high karlovitz number premixed flames, Combust. Flame 176 (2017) 500-510. 
[44] J. F. Driscoll, J. H. Chen, A. W. Skiba, C. D. Carter, E. R. Hawkes, H. Wang, Premixed flames subjected to extreme turbulence: Some questions and recent answers, Prog. Energy Combust. Sci. 76 (2020) 100802.

[45] A. Trouvé, T. Poinsot, The evolution equation for the flame surface density in turbulent premixed combustion, J. Fluid Mech. 278 (1994) 1-31.

[46] T. Poinsot, D. Veynante, Theoretical and numerical combustion, 3rd Edition, Vol. 1, T. Poinsot, S.1., 2012.

[47] D. Veynante, L. Vervisch, Turbulent combustion modeling, Prog. Energy Combust. Sci. 28 (3) (2002) 193-266.

[48] G. Rymer, Analyse et modélisation du taux de réaction moyen et des mécanismes de transport en combustion turbulente prémélangée, Ph.D. thesis, Ec. Centrale de Paris (2001).

[49] T. Echekki, J. H. Chen, Analysis of the contribution of curvature to premixed flame propagation, Combust. Flame 118 (1-2).

[50] V. Moureau, G. Lartigue, Y. Sommerer, C. Angelberger, O. Colin, T. Poinsot, Numerical methods for unsteady compressible multi-component reacting flows on fixed and moving grids, J. Comput. Phys. 202 (2) (2005) 710-736.

[51] F. Tagliante, T. Poinsot, L. M. Pickett, P. Pepiot, L.-M. Malbec, G. Bruneaux, C. Angelberger, A conceptual model of the flame stabilization mechanisms for a lifted diesel-type flame based on direct numerical simulation and experiments, Combust. Flame 201 (2019) 65-77.

[52] O. Colin, M. Rudgyard, Development of high-order taylorgalerkin schemes for les, J. Comput. Phys. 162 (2) (2000) 338371.

[53] A. J. Aspden, M. S. Day, J. B. Bell, Towards the distributed burning regime in turbulent premixed flames, J. Fluid Mech. 871 (2019) 1-21.

[54] A. J. Aspden, M. S. Day, J. B. Bell, Turbulence-chemistry interaction in lean premixed hydrogen combustion, Proc. Combust. Inst. 35 (2) (2015) 1321-1329.

[55] T. Poinsot, S. Lele, Boundary conditions for direct simulations of compressible viscous flows, J. Comput. Phys. 101 (1) (1992) 104-129.

[56] A. Bonhomme, Numerical study of laminar and turbulent flames propagating in a fan-stirred vessel, Ph.D. thesis, Ec. Mec. Energ. Génie civ. procédés (2014).

[57] P. Pepiot-Desjardins, H. Pitsch, An efficient error-propagationbased reduction method for large chemical kinetic mechanisms, Combust. Flame 154 (1-2) (2008) 67-81.

[58] C. Lapeyre, L. Selle, B. Bédat, T. Poinsot, Anr idylle deliverables report, https://clapeyre.github.io/research/.

[59] A. Mzé-Ahmed, K. Hadj-Ali, P. Diévart, P. Dagaut, Kinetics of oxidation of a synthetic jet fuel in a jet-stirred reactor: Experimental and modeling study, Energy \& Fuels 24 (9) (2010) 4904-4911.

[60] V. Eswaran, S. B. Pope, An examination of forcing in direct numerical simulations of turbulence, Comput. Fluids 16 (3) (1988) 257-278.

[61] R. Paoli, K. Shariff, Turbulent condensation of droplets: Direct simulation and a stochastic model, J. Atmos. Sci. 66 (3) (2009) 723-740.

[62] M. Klein, N. Chakraborty, S. Ketterl, A comparison of strategies for direct numerical simulation of turbulence chemistry interaction in generic planar turbulent premixed flames, Flow Turbul. Combust. 99 (3-4) (2017) 955-971.

[63] M. Matalon, C. Cui, J. Bechtold, Hydrodynamic theory of premixed flames: effects of stoichiometry, variable transport coefficients and arbitrary reaction orders, J. Fluid Mech. 487 (2003) 179.

[64] J. Hinze, Turbulence, McGraw-Hill, New York, 2012.

[65] A. J. Aspden, M. S. Day, J. B. Bell, Turbulence-flame interac- tions in lean premixed hydrogen: Transition to the distributed burning regime, J. Fluid Mech. 680 (2011) 287-320.

[66] A. J. Aspden, M. S. Day, J. B. Bell, Lewis number effects in distributed flames, Proc. Combust. Inst. 33 (1) (2011) 1473-1480.

[67] A. J. Aspden, M. S. Day, J. Bell, Three-dimensional direct numerical simulation of turbulent lean premixed methane combustion with detailed kinetics, Combust. Flame 166 (2016) 266283.

[68] A. Aspden, J. Bell, M. Day, F. Egolfopoulos, Turbulence-flame interactions in lean premixed dodecane flames, Proc. Combust. Inst. 36 (2) (2017) 2005-2016.

[69] E. R. Hawkes, O. Chatakonda, H. Kolla, A. R. Kerstein, J. H. Chen, A petascale direct numerical simulation study of the modelling of flame wrinkling for large-eddy simulations in intense turbulence, Combust. Flame 159 (8) (2012) 2690-2703.

[70] A. Poludnenko, E. Oran, The interaction of high-speed turbulence with flames: Global properties and internal flame structure, Combust. Flame 157 (5) (2010) 995-1011.

[71] B. Zhou, C. Brackmann, Z. Wang, Z. Li, M. Richter, M. Aldén, X.-S. Bai, Thin reaction zone and distributed reaction zone regimes in turbulent premixed methane/air flames: Scalar distributions and correlations, Combust. Flame 175 (2017) 220-236.

[72] A. W. Skiba, T. M. Wabel, C. D. Carter, S. D. Hammack, J. E. Temme, J. F. Driscoll, Premixed flames subjected to extreme levels of turbulence part i: Flame structure and a new measured regime diagram, Combust. Flame 189 (2018) 407-432.

[73] R. Sankaran, E. R. Hawkes, J. H. Chen, T. Lu, C. K. Law, Structure of a spatially developing turbulent lean methane-air bunsen flame, Proc. Combust. Inst. 31 (1) (2007) 1291-1298.

[74] K. Pearson, Vii. note on regression and inheritance in the case of two parents, Proc. R. Soc. Lond. 58 (347-352) (1895) 240-242.

[75] N. Chakraborty, R. Cant, Effects of strain rate and curvature on surface density function transport in turbulent premixed flames in the thin reaction zones regime, Phys. Fluids 17 (6) (2005) 065108.

[76] M. Matalon, B. J. Matkowsky, Flames as gasdynamic discontinuities, J. Fluid Mech. 124 (1982) 239-259.

[77] J. H. Chen, H. G. Im, Correlation of flame speed with stretch in turbulent premixed methane/air flames, Symp. (Int.) Combust. 27 (1) (1998) 819-826.

[78] J. B. Chen, H. G. Im, Stretch effects on the burning velocity of turbulent premixed hydrogen/air flames, Proc. Combust. Inst. 28 (1) (2000) 211-218.

[79] P. Clavin, G. Joulin, High-frequency response of premixed flames to weak stretch and curvature: a variable-density analysis, Combust. Theory Model. 1 (4) (1997) 429-446.

[80] E. Knudsen, H. Pitsch, A dynamic model for the turbulent burning velocity for large eddy simulation of premixed combustion, Combust. Flame 154 (4) (2008) 740-760.

[81] L. Vervisch, E. Bidaux, K. Bray, W. Kollmann, Surface density function in premixed turbulent combustion modeling, similarities between probability density function and flame surface approaches, Phys. Fluids 7 (10) (1995) 2496-2503.

[82] R. Cant, S. Pope, K. Bray, Modelling of flamelet surface-tovolume ratio in turbulent premixed combustion, Symp. (Int.) Combust. 23 (1) (1991) 809-815.

[83] C. Meneveau, T. Poinsot, Stretching and quenching of flamelets in premixed turbulent combustion, Combust. Flame 86 (4) (1991) 311-332.

[84] F. Charlette, C. Meneveau, D. Veynante, A power-law flame wrinkling model for les of premixed turbulent combustion part i: Non-dynamic formulation and initial tests, Combust. Flame 131 (1-2) (2002) 159-180.

[85] S. Bougrine, S. Richard, O. Colin, D. Veynante, Fuel composition effects on flame stretch in turbulent premixed combus- 
tion: Numerical analysis of flame-vortex interaction and formulation of a new efficiency function, Flow Turbul. Combust. 93 (2) (2014) 259-281.

[86] Ö. L. Gülder, G. J. Smallwood, Inner cutoff scale of flame surface wrinkling in turbulent premixed flames, Combust. Flame 103 (1-2) (1995) 107-114.

[87] E. R. Hawkes, R. Sankaran, J. H. Chen, An analysis of the flame surface density transport equation using direct numerical simulation of a methane-air jet flame, 2009.

[88] F. Thiesset, F. Halter, C. Bariki, C. Lapeyre, C. Chauveau, I. Gökalp, L. Selle, T. Poinsot, Isolating strain and curvature effects in premixed flame/vortex interactions, J. Fluid Mech. 831 (2017) 618-654.

[89] N. Darabiha, S. M. Candel, F. E. Marble, The effect of strain rate on a premixed laminar flame, Combust. Flame 64 (2) (1986) 203-217.

[90] J. A. van Oijen, Flamelet-generated manifolds: Development and application to premixed laminar flames, Tech. Univ. Eindhoven, 2002. 